JOURNAL OF

SYMPLECTIC GEOMETRY

Volume 5, Number 3, 281-356, 2007

\title{
SYMPLECTIC HYPERSURFACES AND TRANSVERSALITY IN GROMOV-WITTEN THEORY
}

\author{
Kai Cieliebak and Klaus Mohnke
}

We present a new method to prove transversality for holomorphic curves in symplectic manifolds, and show how it leads to a definition of genus zero Gromov-Witten invariants. The main idea is to introduce additional marked points that are mapped to a symplectic hypersurface of high degree in order to stabilize the domains of holomorphic maps.

Dedicated to Dusa McDuff

\section{Contents}

1. Introduction 281

2. Nodal curves $\quad 285$

3. Coherent almost complex structures $\quad 289$

4. Holomorphic maps $\quad 297$

5. Nodal maps $\quad 300$

6. Tangencies $\quad 310$

7. Intersection numbers $\quad 321$

8. Symplectic hypersurfaces $\quad 324$

9. Proof of transversality 342

10. Independence of the auxiliary data 352

References $\quad 355$

\section{Introduction}

Donaldson's construction of symplectic hypersurfaces [4] and its extensions lead to a new method to prove transversality for holomorphic curves in symplectic manifolds. In this paper, we illustrate this method for holomorphic 
spheres in closed symplectic manifolds, and show how it leads to a definition of genus zero Gromov-Witten invariants.

The transversality question in Gromov-Witten theory has previously been discussed by many authors; see, e.g., $[12,16,17,19,20,22]$ and further references therein. Transversality for $J$-holomorphic disks with Lagrangian boundary conditions is discussed in [11]. A very general approach is being developed by Hofer, Wysocki and Zehnder. Their "polyfold Fredholm theory" provides a framework to study transversality for solution spaces of elliptic PDEs, which may have applications far beyond symplectic geometry. By contrast our approach, being specifically designed for holomorphic curves, appears to be much simpler in this case. In particular, we use only standard tools from functional analysis and our moduli spaces (in the genus zero case) are smooth finite-dimensional manifolds.

We now state our results. Consider a closed symplectic manifold $\left(X^{2 n}, \omega\right)$ of dimension $2 n$ such that $\omega$ represents an integral cohomology class $[\omega] \in H^{2}(X ; \mathbb{Z})$. Recall that an almost complex structure $J$ on $X$ is called $\omega$-tamed if $\omega(v, J v)>0$ for all tangent vectors $v$, and $\omega$-compatible if, in addition, the bilinear form $\omega(\cdot, J \cdot)$ is symmetric (hence a Riemannian metric). Our construction uses as auxiliary datum a hypersurface (i.e., a closed codimension 2 submanifold) $Y \subset X$, representing the homology class Poincaré dual to $D[\omega]$ for some degree $D \in \mathbb{N}$, whose Kähler angle (see Section 8) with respect to a compatible pair $(\omega, J)$ satisfies $\theta(Y ; \omega, J)<\theta_{3}$. Here $0<\theta_{3}<\theta_{1}<1$ are universal constants depending only on the dimension. In particular, the hypersurface $Y$ is symplectic. By a result of Donaldson [4], for a given $\omega$-compatible $J$ such a hypersurface $Y$ exists for each sufficiently large $D \in \mathbb{N}$. We will refer to such $(J, Y)$ as a Donaldson pair if, in addition, $D \geq D^{*}(X, \omega, J)$ for a suitable positive constant $D^{*}$ depending on $(X, \omega, J)$ (see Definition 9.2).

Given a Donaldson pair $(J, Y)$, we denote by $\mathcal{J}\left(X, Y ; J, \theta_{1}\right)$ the space of $\omega$-tamed almost complex structures on $X$ which leave $T Y$ invariant and are $\theta_{1}$-close to $J$ in the $C^{0}$-norm. For $\ell \geq 3$ we define a class

$$
\mathcal{J}_{\ell+1}\left(X, Y ; J, \theta_{1}\right) \subset C^{\infty}\left(\overline{\mathcal{M}}_{\ell+1}, \mathcal{J}\left(X, Y ; J, \theta_{1}\right)\right)
$$

of almost complex structures depending smoothly on points in the DeligneMumford space $\overline{\mathcal{M}}_{\ell+1}$ of stable genus zero curves with $\ell+1$ marked points $z_{0}, \ldots, z_{\ell}$. Moreover, we require elements in $\mathcal{J}_{\ell+1}\left(X, Y ; J, \theta_{1}\right)$ to be coherent under gluing of strata in $\overline{\mathcal{M}}_{\ell+1}$, see Section 3 for the precise definition.

Via the canonical projection $\pi: \overline{\mathcal{M}}_{\ell+1} \rightarrow \overline{\mathcal{M}}_{\ell}$ forgetting the marked point $z_{0}$, we think of $K \in \mathcal{J}_{\ell+1}\left(X, Y ; J, \theta_{1}\right)$ as a collection of almost complex structures $K_{\mathbf{z}} \in \mathcal{J}\left(X, Y ; J, \theta_{1}\right)$, parametrized by $\mathbf{z} \in \overline{\mathcal{M}}_{\ell}$, depending smoothly on $z_{0}$ thought of as a point on the nodal Riemann surface $\pi^{-1}(\mathbf{z})$ defined by z. To $K \in \mathcal{J}_{\ell+1}\left(X, Y ; J, \theta_{1}\right)$ and a stable map $(\mathbf{z}, \mathbf{f})$ with $\ell$ marked points we 


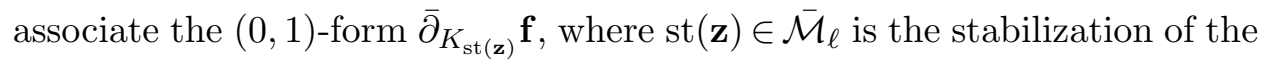
underlying nodal curve $\mathbf{z}$. We call $(\mathbf{z}, \mathbf{f}) K$-holomorphic if $\bar{\partial}_{K_{\mathrm{st}(\mathbf{z})}} \mathbf{f}=0$.

Denote by $c_{1}$ the first Chern class of $X$. For a homology class $A \in H_{2}(X ; \mathbb{Z})$ set $\ell:=D \omega(A)$. In addition, fix an integer $k \geq 0$. Every $K \in \mathcal{J}_{\ell+1}\left(X, Y ; J, \theta_{1}\right)$ induces elements $\pi_{\ell}^{*} K \in \mathcal{J}_{\ell+k+1}\left(X, Y ; J, \theta_{1}\right)$ by composition with the map $\pi_{\ell}: \overline{\mathcal{M}}_{k+\ell+1} \rightarrow \overline{\mathcal{M}}_{\ell+1}$ that forgets the first $k$ marked points (and stabilizes). Using this, we define the moduli space $\mathcal{M}_{k+\ell}(A, K ; Y)$ of (smooth) $\pi_{\ell}^{*} K$ holomorphic spheres in the class $A$ with $k+\ell$ marked points and mapping the last $\ell$ marked points to $Y$.

More generally, consider a $(k+\ell)$-labelled tree $T$ which is $\ell$-stable, i.e., stable after removing the first $k$ marked points (see Section 2). For homology classes $A_{\alpha}$ corresponding to vertices $\alpha$ of $T$ denote by $\mathcal{M}_{T}\left(\left\{A_{\alpha}\right\}, K ; Y\right)$ the space of stable $K$-holomorphic spheres modelled over $T$, representing the homology classes $A_{\alpha}$ and and mapping the last $\ell$ marked points to $Y$. We denote by ghost tree a maximal subtree $T^{\prime} \subset T$ such that $A_{\alpha}=0$ for all $\alpha \in T^{\prime}$, so the corresponding holomorphic maps are constant on $T^{\prime}$. Our first result is

Theorem 1.1. Let $(X, \omega)$ be a closed symplectic manifold such that $[\omega] \in H^{2}(X ; \mathbb{Z})$. Then for every Donaldson pair $(J, Y)$ of degree $D \geq$ $D^{*}(X, \omega, J)$ and integer multiples $\ell \geq 3$ of $D$ there exist nonempty sets $\mathcal{J}_{\ell+1}^{\mathrm{reg}}\left(X, Y ; J, \theta_{1}\right) \subset \mathcal{J}_{\ell+1}\left(X, Y ; J, \theta_{1}\right)$ such that for $K \in \mathcal{J}_{\ell+1}^{\mathrm{reg}}\left(X, Y ; J, \theta_{1}\right)$ the following holds. Let $A \in H_{2}(X ; \mathbb{Z})$ be a homology class with $\ell=D \omega(A)$. For $k \geq 0$ let $T$ be an $\ell$-stable $(k+\ell)$-labelled tree with $e(T)$ edges, and $A_{\alpha} \in H_{2}(X ; \mathbb{Z})$ for $\alpha \in T$ with $\sum A_{\alpha}=A$, such that every ghost tree contains at most one of the last $\ell$ marked points. Then the moduli space $\mathcal{M}_{T}\left(\left\{A_{\alpha}\right\}, K ; Y\right)$ of stable $K$-holomorphic spheres modelled over $T$, representing the homology classes $A_{\alpha}$ and mapping the last $\ell$ marked points to $Y$, is a smooth manifold of real dimension

$$
\operatorname{dim}_{\mathbb{R}} \mathcal{M}_{T}\left(\left\{A_{\alpha}\right\}, K ; Y\right)=2\left(n-3+k+c_{1}(A)-e(T)\right) .
$$

To interpret this result, recall from $[\mathbf{1 9}]$ the definition of a pseudocycle. We say that a subset $\Omega \subset X$ of a manifold $X$ has dimension at most $d$ if $\Omega$ is contained in the image of a smooth map $g: N \rightarrow X$ from a manifold of dimension $\operatorname{dim} N \leq d$. A smooth map $f: M \rightarrow X$ from an oriented $d$ manifold $M$ to a manifold $X$ is called d-dimensional pseudocycle if $f(M)$ has compact closure and its "omega limit set"

$$
\Omega_{f}:=\bigcap_{K \subset M \text { compact }} \overline{f(M \backslash K)}
$$

has dimension at most $d-2$. Thus intuitively, $f(M)$ can be compactified by adding strata of codimension at least 2 . 
Our second result, proved in Section 9, will be a consequence of Theorem 1.1 and an appropriate version of Gromov compactness.

Theorem 1.2. In the notation of Theorem 1.1, for every $k \geq 1$ the evaluation map at the first $k$ points

$$
\mathrm{ev}^{k}: \mathcal{M}_{k+\ell}(A, K ; Y) \longmapsto X^{k}
$$

represents a pseudocycle $\mathrm{ev}^{k}(J, Y ; K)$ of dimension

$$
2 d:=2\left(n-3+k+c_{1}(A)\right) .
$$

The third result concerns the dependence of this pseudocycle on the auxiliary data $(J, Y ; K)$. Two pseudocycles $f_{i}: M_{i} \rightarrow X, i=0,1$, are called cobordant if there exists a smooth map $F: W \rightarrow X$ from an oriented $(d+1)$-manifold $W$ with boundary $\partial W=M_{1}-M_{0}$ such that $\left.F\right|_{M_{i}}=f_{i}$ and $\operatorname{dim} \Omega_{F} \leq d-1$. Let a rational pseudocycle be a pseudocycle multiplied with a positive rational weight. For pseudocycles $f_{0}, f_{1}$ we say that $f_{0}$ and $\ell f_{1}$ are equal as currents if there exists a covering map $\phi: M_{0} \rightarrow M_{1}$ of degree $\ell$ such that $f_{0}=f_{1} \circ \phi$. We denote by rational cobordism the equivalence relation on rational pseudocycles generated by equality as currents and cobordism of pseudocycles.

The following result, proved in Section 10, will be a consequence of the asymptotic uniqueness [2] of Donaldson hypersurfaces.

Theorem 1.3. Up to rational cobordism, the rational pseudocycle $\frac{1}{\ell^{!}} \mathrm{ev}^{k}(J, Y ; K)$ in Theorem 1.2 does not depend on the choice of the auxiliary data $(J, Y ; K)$.

Following the strategy of [19], Theorems 1.2 and 1.3 allow us to define Gromov-Witten invariants for arbitrary closed symplectic manifolds $(X, \omega)$ as follows. Let $A \in H_{2}(X ; \mathbb{Z})$ and let $\alpha_{1}, \ldots, \alpha_{k}$ be nontorsion integral cohomology classes on $X$ of total degree

$$
\sum_{i=1}^{k} \operatorname{deg}\left(\alpha_{i}\right)=2 d=2 n-6+2 k+2 c_{1}(A) .
$$

Represent the Poincaré dual of $\pi_{1}^{*} \alpha_{1} \cup \cdots \cup \pi_{k}^{*} \alpha_{k} \in H^{*}\left(X^{k}\right)$ by a cycle $a$ in $X^{k}$ that is strongly transverse in the sense of [19] to the evaluation map $\operatorname{ev}^{k}(J, Y ; K): \mathcal{M}_{k+\ell}(A, J ; Y) \mapsto X^{k}$ from Theorem 1.2. Define the GromovWitten invariant of holomorphic spheres passing through cycles dual to $\alpha_{1}, \ldots, \alpha_{k}$ as the intersection number

$$
\mathrm{GW}_{A}\left(\alpha_{1}, \ldots, \alpha_{k}\right):=\frac{1}{\ell !} \operatorname{ev}^{k}(J, Y ; K) \cdot a \in \mathbb{Q},
$$

with $\ell=D \omega(A)$ as in Theorem 1.1. In view of Theorem 1.3, this number does not depend on the choice of $(J, Y ; K)$ and by Theorem 1.2 not on the choice of $a$. 
Remark 1.4. The method described in this paper is applicable to prove transversality for more general holomorphic curves. The extension to genus zero holomorphic curves with boundary on a Lagrangian submanifold as in Floer homology [11] relies on the existence of approximately holomorphic hypersurfaces in the complement of a Lagrangian submanifold due to Auroux, Gayet and Mohsen [3], and the extension to genus zero holomorphic curves with punctures asymptotic to closed Reeb orbits as in symplectic field theory [8] is based on the existence of contact open books due to Giroux [13]. Both cases also require good descriptions of the relevant Deligne-Mumford spaces of stable genus zero curves (in the spirit of Section 3): curves with marked points and boundary in the first case, and curves with marked points and directions at the marked points in the second case. Note that in both cases the moduli spaces have codimension 1 boundary, so one needs to prove appropriate gluing results. These extensions may be treated in subsequent papers.

Remark 1.5. This paper is clearly related to the work of Ionel and Parker on relative Gromov-Witten invariants $[\mathbf{1 4}, \mathbf{1 5}]$. Indeed, moduli spaces of holomorphic curves with tangency conditions to one or more symplectic hypersurfaces are the central objects of study in their as well as our work. However, the motivations and goals are very different. In particular, we do not define relative Gromov-Witten invariants (although this should be possible using the techniques developed in this paper).

Remark 1.6. One may wonder how the Gromov-Witten invariants defined in this paper are related to other definitions. First, we claim that if the symplectic manifold $(X, \omega)$ is semipositive in the sense of McDuff and Salamon [19], then our definition agrees with the one given in [19]. This essentially follows from the fact that in that case we can choose our perturbation $K$ domain-independent. On the other hand, the comparison of our definition with ones involving abstract perturbations and virtual moduli cycles as in $[\mathbf{1 2}, \mathbf{1 6}, \mathbf{1 7}, \mathbf{2 0}, \mathbf{2 2}]$ lies beyond the scope of this paper, whose purpose is exactly to avoid abstract perturbations. However, taking virtual moduli cycles for granted, our invariant seems to coincide with the relative Gromov-Witten invariant in $[\mathbf{1 4}, \mathbf{1 5}]$, which in turn equals the absolute Gromov-Witten invariant according to Proposition 14.9.

\section{Nodal curves}

In this section, we describe the space $\overline{\mathcal{M}}_{k}$ of stable curves of genus zero with $k$ marked points. We adopt the approach and notation of [19].

A $k$-labelled tree is a triple $T=(T, E, \Lambda)$, where $(T, E)$ is a (connected) tree with set of vertices $T$ and edge relation $E \subset T \times T$, and $\Lambda=\left\{\Lambda_{\alpha}\right\}_{\alpha \in T}$ 
is a decomposition of the index set $\{1, \ldots, k\}=\amalg_{\alpha \in T} \Lambda_{\alpha}$. We write $\alpha E \beta$ if $(\alpha, \beta) \in E$. Note that the labelling $\Lambda$ defines a unique map $\{1, \ldots, k\} \rightarrow T$, $i \mapsto \alpha_{i}$ by the requirement $i \in \Lambda_{\alpha_{i}}$. Let

$$
e(T)=|T|-1
$$

be the number of edges. A tree homomorphism $\tau: T \rightarrow \tilde{T}$ is a map which collapses some subtrees of $T$ to vertices of $\tilde{T}$ (cf. [19]). A tree homomorphism $\tau$ is called tree isomorphism if it is bijective and $\tau^{-1}$ is a tree homomorphism.

A tree $T$ is called stable if for each $\alpha \in T$,

$$
n_{\alpha}:=\# \Lambda_{\alpha}+\#\{\beta \mid \alpha E \beta\} \geq 3 \text {. }
$$

Note that for $k<3$ every $k$-labelled tree is unstable. For $k \geq 3$, a $k$-labelled tree $T$ can be stabilized in a canonical way to a stable $k$-labelled tree $\operatorname{st}(T)$ by deleting the vertices with $n_{\alpha}<3$ and modifying the edges in the obvious way (cf. [19]).

A nodal curve of genus zero with $k$ marked points modelled over the tree $T=(T, E, \Lambda)$ is a tuple

$$
\mathbf{z}=\left(\left\{z_{\alpha \beta}\right\}_{\alpha E \beta},\left\{z_{i}\right\}_{1 \leq i \leq k}\right)
$$

of points $z_{\alpha \beta}, z_{i} \in S^{2}$ such that for each $\alpha \in T$ the special points

$$
\mathrm{SP}_{\alpha}:=\left\{z_{\alpha \beta} \mid \alpha E \beta\right\} \cup\left\{z_{i} \mid \alpha_{i}=\alpha\right\}
$$

are pairwise distinct. For $\alpha \in T$ and $i \in\{1, \ldots, k\}$ denote by $z_{\alpha i}$ either the point $z_{i}$ if $i \in \Lambda_{\alpha}$ or the point $z_{\alpha \beta_{1}}$ if $z_{i} \in \Lambda_{\beta_{r}}$ and $\left(\alpha, \beta_{1}\right),\left(\beta_{1}, \beta_{2}\right), \ldots$, $\left(\beta_{r-1}, \beta_{r}\right) \in E$. Note that $n_{\alpha}=\# \mathrm{SP}_{\alpha}$. We associate to $\mathbf{z}$ the nodal Riemann surface

$$
\Sigma_{\mathbf{z}}:=\coprod_{\alpha \in T} S_{\alpha} / z_{\alpha \beta} \sim z_{\beta \alpha}
$$

obtained by gluing a collection of standard spheres $\left\{S_{\alpha}\right\}_{\alpha \in T}$ at the points $z_{\alpha \beta}$ for $\alpha E \beta$, with marked points $z_{i} \in S_{\alpha_{i}}, i=1, \ldots, k$. Note that $\mathbf{z}$ can be uniquely recovered from $\Sigma_{\mathbf{z}}$, so we will sometimes not distinguish between the two. A nodal curve $\mathbf{z}$ is called stable if the underlying tree is stable, i.e., every sphere $S_{\alpha}$ carries at least three special points. Stabilization of trees induces a canonical stabilization of nodal curves $\mathbf{z} \mapsto \operatorname{st}(\mathbf{z})$.

We will usually omit the genus zero from the notation. Denote the space of all nodal curves (of genus zero) with $k$ marked points modelled over $T$ by

$$
\tilde{\mathcal{M}}_{T} \subset\left(S^{2}\right)^{E} \times\left(S^{2}\right)^{k}
$$


Note that this is an open subset of the product of spheres. A morphism between nodal curves $\mathbf{z}, \tilde{\mathbf{z}}$ modelled over trees $T, \tilde{T}$ is a tuple

$$
\phi=\left(\tau,\left\{\phi_{\alpha}\right\}_{\alpha \in T}\right),
$$

where $\tau: T \rightarrow \tilde{T}$ is a tree homomorphism and $\phi_{\alpha}: S^{2} \cong S_{\alpha} \rightarrow S_{\tau(\alpha)} \cong S^{2}$ are (possibly constant) holomorphic maps such that

$$
\begin{aligned}
\tilde{z}_{\tau(\alpha) \tau(\beta)}=\phi_{\alpha}\left(z_{\alpha \beta}\right) & \text { if } \tau(\alpha) \neq \tau(\beta), \\
\phi_{\alpha}\left(z_{\alpha \beta}\right)=\phi_{\beta}\left(z_{\beta \alpha}\right) & \text { if } \tau(\alpha)=\tau(\beta), \\
\tilde{\alpha}_{i}=\tau\left(\alpha_{i}\right), & \tilde{z}_{i}=\phi_{\alpha_{i}}\left(z_{i}\right)
\end{aligned}
$$

for $i=1, \ldots, k$ and $\alpha E \beta$. A morphism $\phi: \mathbf{z} \rightarrow \tilde{\mathbf{z}}$ induces a natural holomorphic map $\Sigma_{\mathbf{z}} \rightarrow \Sigma_{\tilde{\mathbf{z}}}$ (i.e., a continuous map that is holomorphic on each component $\left.S_{\alpha}\right)$ which we deliberately denote again by $\phi$. A morphism $\left(\tau,\left\{\phi_{\alpha}\right\}\right)$ is called isomorphism if $\tau$ is a tree isomorphism and each $\phi_{\alpha}$ is biholomorphic.

Isomorphisms from $\mathbf{z}$ to itself are called automorphisms. If $\mathbf{z}$ is stable its only automorphism is the identity (see [19], discussion after Definition D.3.4). Thus for a stable tree $T$ we have a free and proper holomorphic action

$$
G_{T} \times \tilde{\mathcal{M}}_{T} \longrightarrow \tilde{\mathcal{M}}_{T}
$$

of the group $G_{T}$ of isomorphisms fixing $T$.

Remark 2.1. Properness follows directly from the characterization of sequences of Möbius transforms which do not have a uniformly convergent subsequence in [19, Lemma D.1.2]. Indeed, if $\mathbf{z}^{\nu} \rightarrow \mathbf{z}$ and $\phi^{\nu}\left(\mathbf{z}^{\nu}\right) \rightarrow \mathbf{z}^{\prime}$ in $\tilde{\mathcal{M}}_{T}$ as $\nu \rightarrow \infty$ and $\left\{\phi^{\nu}\right\}$ has no uniformly convergent subsequence in $G_{T}$, then there is an $\alpha \in T$ and a subsequence $\phi^{\nu^{\prime}}$ which converges to the constant map $S_{\alpha} \rightarrow\{y\}$ on compact subsets of $S_{\alpha} \backslash\{x\}$ for some points $x, y \in S_{\alpha}$. In particular, the limit of the sets $\left\{\phi_{\alpha}^{\nu^{\prime}}\left(z_{i}^{\nu^{\prime}}\right), \phi_{\alpha}^{\nu^{\prime}}\left(z_{\alpha \beta}^{\nu^{\prime}}\right) \mid z_{i} \in \Lambda_{\alpha}, \alpha E \beta\right\}$ consists of at most two points, contradicting $\phi^{\nu}\left(\mathbf{z}^{\nu}\right) \rightarrow \mathbf{z}^{\prime}$ and the stability of $\mathbf{z}^{\prime}$.

Hence the quotient

$$
\mathcal{M}_{T}:=\tilde{\mathcal{M}}_{T} / G_{T}
$$

is a complex manifold of dimension

$$
\operatorname{dim}_{\mathbb{C}} \mathcal{M}_{T}=k+2 e(T)-3|T|=k-3-e(T) .
$$

For $k \geq 3$, denote by $\mathcal{M}_{k}=\tilde{\mathcal{M}}_{k} / G$ the moduli space of stable curves modelled over the $k$-labelled tree with one vertex. As a set, the Deligne-Mumford space (of genus zero) with $k$ marked points is given by

$$
\overline{\mathcal{M}}_{k}:=\coprod_{T} \mathcal{M}_{T},
$$


where the union is taken over the (finitely many) isomorphism classes of stable $k$-labelled trees. However, $\overline{\mathcal{M}}_{k}$ is equipped with the topology of Gromov convergence which makes it a compact connected metrizable space (see [19]). As the notation suggests, $\overline{\mathcal{M}}_{k}$ is the compactification of $\mathcal{M}_{k}$ in the Gromov topology. For a stable $k$-labelled tree $T$, the closure of $\mathcal{M}_{T}$ in $\overline{\mathcal{M}}_{k}$ is given by

$$
\overline{\mathcal{M}}_{T}=\coprod_{\tilde{T}} \mathcal{M}_{\tilde{T}}
$$

where the union is taken over all isomorphism classes of stable $k$-labelled trees $\tilde{T}$ for which there exists a surjective tree homomorphism $\tau: \tilde{T} \rightarrow T$ with $\tau\left(\tilde{\alpha}_{i}\right)=\alpha_{i}$ for $i=1, \ldots, k$.

We have the following result of Knudsen (cf. [19]).

Theorem 2.2. For $k \geq 3$, the Deligne-Mumford space $\overline{\mathcal{M}}_{k}$ is a compact complex manifold of dimension $\operatorname{dim}_{\mathbb{C}} \overline{\mathcal{M}}_{k}=k-3$. Moreover, for each stable $k$-labelled tree $T$, the space $\overline{\mathcal{M}}_{T} \subset \overline{\mathcal{M}}_{k}$ is a compact complex submanifold of codimension codim $_{\mathbb{C}} \overline{\mathcal{M}}_{T}=e(T)$.

Remark 2.3. In this paper we do not use the complex structure on $\overline{\mathcal{M}}_{k}$, but only its structure as a smooth manifold.

We have a canonical projection $\pi: \overline{\mathcal{M}}_{k+1} \rightarrow \overline{\mathcal{M}}_{k}$ by forgetting the $(k+1)$ st marked point and stabilizing. The map $\pi$ is holomorphic and the fibre $\pi^{-1}([\mathbf{z}])$ is naturally biholomorphic to $\Sigma_{\mathbf{z}}$. The projection $\pi: \overline{\mathcal{M}}_{k+1} \rightarrow \overline{\mathcal{M}}_{k}$ is called the universal curve.

Lemma 2.4. For $[\mathbf{z}] \in \overline{\mathcal{M}}_{k}$, every component $S_{\alpha}$ of $\Sigma_{\mathbf{z}} \cong \pi^{-1}([\mathbf{z}])$ is an embedded holomorphic sphere in $\overline{\mathcal{M}}_{k+1}$.

Proof. This follows from the construction of coordinate charts on $\overline{\mathcal{M}}_{k+1}$ in terms of cross ratios in [19] and the fact that any nonconstant cross ratio maps $S_{\alpha}$ biholomorphically onto $\mathbb{C} P^{1}$.

The following two lemmas follow directly from the construction of the stabilization.

Lemma 2.5. Let $\mathbf{z}$ be a nodal curve modelled over the tree $T$ and $\operatorname{st}(\mathbf{z})$ its stabilization. Then the stabilization map induces a morphism

$$
\text { st }=\left(\tau,\left\{\phi_{\alpha}\right\}_{\alpha \in T}\right): \Sigma_{\mathbf{z}} \longmapsto \Sigma_{\mathrm{st}(\mathbf{z})}
$$

with the following property. There exists a collection of subtrees $T^{\prime} \subset T$ such that $\phi_{\alpha}$ is constant for $\alpha \in T^{\prime}$ and biholomorphic otherwise. Moreover, each sphere $S_{\alpha}$ with $\alpha \notin T^{\prime}$ carries at least three special points. 
Lemma 2.6. The projection $\pi_{\ell}:=\pi \circ \cdots \circ \pi: \overline{\mathcal{M}}_{k+\ell} \rightarrow \overline{\mathcal{M}}_{\ell}$ induces for each $[\mathbf{z}] \in \overline{\mathcal{M}}_{k+\ell}$ and $[\tilde{\mathbf{z}}]:=\pi_{\ell}[\mathbf{z}]$ a morphism

$$
\pi_{\ell}=\left(\tau,\left\{\phi_{\alpha}\right\}_{\alpha \in T}\right): \Sigma_{\mathbf{z}} \longmapsto \Sigma_{\tilde{\mathbf{z}}}
$$

with the following property. There exists a collection of subtrees $T^{\prime} \subset T$ such that $\phi_{\alpha}$ is constant for $\alpha \in T^{\prime}$ and biholomorphic otherwise.

\section{Coherent almost complex structures}

Coherent maps. Now we consider the Deligne-Mumford space $\overline{\mathcal{M}}_{k+1}$ with $k+1$ marked points $z_{0}, \ldots, z_{k}$. In the following discussion, the point $z_{0}$ plays a special role (it will be the variable for holomorphic maps in later sections).

Given a stable $(k+1)$-labelled tree $T$, we define an equivalence relation on $\{0, \ldots, k\}$ by $i \sim j$ iff $z_{\alpha_{0} i}=z_{\alpha_{0} j}$. The equivalence classes yield a decomposition

$$
\{0, \ldots, k\}=I_{0} \cup \cdots \cup I_{\ell} .
$$

Note that the marked points on $S_{\alpha_{0}}$ correspond to equivalence classes consisting of one element; in particular, we may put $I_{0}:=\{0\}$. Stability implies $\ell+1=n_{\alpha_{0}} \geq 3$. Conversely, we call a decomposition $\mathbf{I}=\left(I_{0}, \ldots, I_{\ell}\right)$ of $\{0, \ldots, k\}$ stable if $I_{0}=\{0\}$ and $|\mathbf{I}|:=\ell+1 \geq 3$. We will always order the $I_{j}$ such that the integers $i_{j}:=\min \left\{i \mid i \in I_{j}\right\}$ satisfy

$$
0=i_{0}<i_{1}<\cdots<i_{\ell}
$$

Denote by $\mathcal{M}_{\mathbf{I}}=\mathcal{M}_{\left(I_{0}, \ldots, I_{\ell}\right)} \subset \overline{\mathcal{M}}_{k+1}$ the union over those stable trees that give rise to the stable decomposition $\mathbf{I}=\left(I_{0}, \ldots, I_{\ell}\right)$. The $\mathcal{M}_{\mathbf{I}}$ are submanifolds of $\overline{\mathcal{M}}_{k+1}$ with

$$
\overline{\mathcal{M}}_{k+1}=\bigcup_{\mathbf{I}} \mathcal{M}_{\mathbf{I}}
$$

and the closure of $\mathcal{M}_{\mathbf{J}}$ is a union of certain strata $\mathcal{M}_{\mathbf{I}}$ with $|\mathbf{I}| \leq|\mathbf{J}|$. The above ordering of the $I_{j}$ determines a projection

$$
p_{\mathbf{I}}: \mathcal{M}_{\mathbf{I}} \longrightarrow \mathcal{M}_{|\mathbf{I}|},
$$

sending a stable curve $\mathbf{z}$ to the special points on the component $S_{\alpha_{0}}$.

Definition 3.1. Let $Z$ be a Banach space and $k \geq 3$. We call a continuous map $F: \overline{\mathcal{M}}_{k+1} \rightarrow Z$ coherent if it satisfies the following two conditions:

(a) $F \equiv 0$ in a neighbourhood of those $\mathcal{M}_{\mathbf{I}}$ with $|\mathbf{I}|=3$;

(b) for every stable decomposition $\mathbf{I}$ with $|\mathbf{I}| \geq 4$ there exists a smooth map $F_{\mathbf{I}}: \mathcal{M}_{|\mathbf{I}|} \rightarrow Z$ such that

$$
\left.F\right|_{\mathcal{M}_{\mathbf{I}}}=F_{\mathbf{I}} \circ p_{\mathbf{I}}: \mathcal{M}_{\mathbf{I}} \longrightarrow Z \text {. }
$$


More generally, let $Z^{*} \subset Z$ be an open neighbourhood of 0 and let $\mathcal{I}$ be a collection of stable decompositions. Then we call a continuous map $F: \cup_{\mathbf{I} \in \mathcal{I}} \mathcal{M}_{\mathbf{I}} \rightarrow Z^{*}$ coherent if it satisfies conditions (a) and (b) and in addition:

(c) The image of $F$ is contractible in $Z^{*}$.

We denote the space of coherent maps from $\overline{\mathcal{M}}_{k+1}$ to $Z$, respectively,

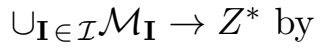

$$
\operatorname{Coh}\left(\overline{\mathcal{M}}_{k+1}, Z\right) \quad \text { respectively } \operatorname{Coh}\left(\bigcup_{\mathbf{I} \in \mathcal{I}} \mathcal{M}_{\mathbf{I}}, Z^{*}\right)
$$

It is equipped with the $C^{0}$-topology on $\overline{\mathcal{M}}_{k+1}$ and the $C^{\infty}$-topology on each $\mathcal{M}_{\mathbf{I}}$ via the projection $p_{\mathbf{I}}$.

Remark 3.2. Condition (a) ensures that almost complex structures which depend in a coherent way on the domain will coincide with a structure which is independent of the domain near double points. It is included to keep the analysis of gluing holomorphic curves as simple as possible (although gluing is not studied in this paper).

Our constructions of coherent maps will be based on the following.

Proposition 3.3. Let $Z$ be a Banach space and $k \geq 3$. For each stable decomposition $\mathbf{I}$ with $|\mathbf{I}| \geq 4$ fix an open subset $U_{\mathbf{I}} \subset \mathcal{M}_{|\mathbf{I}|}$ with compact closure. Then there exist continuous linear extension maps

$$
E_{\mathbf{I}}: C_{0}^{\infty}\left(U_{\mathbf{I}}, Z\right) \longrightarrow \operatorname{Coh}\left(\overline{\mathcal{M}}_{k+1}, Z\right)
$$

such that

$$
\left.\left(E_{\mathbf{I}} \xi\right)\right|_{\mathcal{M}_{\mathbf{I}}}=\xi \circ p_{\mathbf{I}}
$$

for every $\xi \in C_{0}^{\infty}\left(U_{\mathbf{I}}, Z\right)$. Moreover, the extension operators can be chosen such that $E_{\mathbf{I}} \xi$ and $E_{\mathbf{J}} \eta$ have disjoint supports for all $\xi \in C_{0}^{\infty}\left(U_{\mathbf{I}}, Z\right)$ and $\eta \in C_{0}^{\infty}\left(U_{\mathbf{J}}, Z\right)$ with $\mathbf{I} \neq \mathbf{J}$. If $Z^{*} \subset Z$ is open and star-shaped with respect to the origin, then $E_{\mathbf{I}}$ maps $C_{0}^{\infty}\left(U_{\mathbf{I}}, Z^{*}\right)$ to $\operatorname{Coh}\left(\overline{\mathcal{M}}_{k+1}, Z^{*}\right)$.

The proof of Proposition 3.3 will be given below. We first give a reformulation of the coherency condition.

Cross ratios. For pairwise distinct points $z_{0}, z_{1}, z_{2}, z_{3} \in S^{2}=\mathbb{C} \cup\{\infty\}$, we have their cross ratio

$$
w\left(z_{0}, z_{1}, z_{2}, z_{3}\right):=\frac{\left(z_{1}-z_{2}\right)\left(z_{3}-z_{0}\right)}{\left(z_{0}-z_{1}\right)\left(z_{2}-z_{3}\right)} \in S^{2} \backslash\{0,1, \infty\} .
$$


It extends to the cases where two (but no three) of the $z_{i}$ are equal with the values $0,1, \infty$. For $0 \leq i<j<m<n \leq k$ define cross ratios $w_{i j m n}(\mathbf{z}):=w\left(z_{i}, z_{j}, z_{m}, z_{n}\right)$ on $\mathcal{M}_{k+1}$. We will need the following two facts, of which the first one is obvious and the second and third ones are proved in $[\mathbf{1 9}]$.

Fact 1. The cross ratios $w_{0,1,2, j}$ with $3 \leq j \leq k$ define an embedding

$$
\phi_{k+1}: \mathcal{M}_{k+1} \hookrightarrow\left(S^{2}\right)^{k-2}
$$

Fact 2. Each cross ratio $w_{i j m n}, 0 \leq i<j<m<n \leq k$, extends to a continuous function $w_{i j m n}: \overline{\mathcal{M}}_{k+1} \rightarrow S^{2}$; on a stable curve $\mathbf{z}$ modelled over a tree $T$ it is given by $w_{i j m n}(\mathbf{z}):=w\left(z_{\alpha i}, z_{\alpha j}, z_{\alpha m}, z_{\alpha n}\right)$, where $\alpha \in T$ is a vertex for which no three of the points $z_{\alpha i}, z_{\alpha j}, z_{\alpha m}, z_{\alpha n}$ are equal. (This vertex $\alpha$ need not be unique; however, if it is not then there are precisely two such vertices and both choices give the same value in $\{0,1, \infty\}$ for the cross ratio).

Fact 3. $\overline{\mathcal{M}}_{k+1} \backslash \mathcal{M}_{k+1}$ is precisely the locus where some cross ratio $w_{0 i j m}$, $1 \leq i<j<m \leq k$, takes values in $\{0,1, \infty\}$.

Remark 3.4. In fact, it is shown in [19] that the cross ratios $w_{i j m n}$ for $0 \leq i<j<m<n \leq k$ define an embedding

$$
\overline{\mathcal{M}}_{k+1} \hookrightarrow\left(S^{2}\right)^{\left(\begin{array}{c}
k+1 \\
4
\end{array}\right)} .
$$

But we will not use this result in this paper.

Let $\mathbf{I}=\left(I_{0}, \ldots, I_{\ell}\right)$ be a stable decomposition. Recall that $i, j$ are equivalent iff they belong to the same $I_{a}$ for some $1 \leq a \leq \ell$. We say that with respect to the decomposition $\mathbf{I}$ a triple $(i, j, m)$ with $1 \leq i<j<m \leq k$ is of

- Type I: if $i, j, m$ are pairwise nonequivalent;

- Type II: if exactly two of the $i, j, m$ are equivalent;

- Type III: if $i, j, m$ are all equivalent.

We define the type of a cross ratio $\mathbf{w}_{0, i, j, m}$ as the type of the triple $(i, j, m)$. For two stable decompositions $\mathbf{I}, \mathbf{J}$ we say that $\mathbf{J}$ is a refinement of $\mathbf{I}$ if $\mathbf{J}$-equivalence implies I-equivalence, in other words every $J_{b}$ is contained in some $I_{a}$. Note that $\mathbf{J}$ is a refinement of $\mathbf{I}$ if and only if $\mathcal{M}_{\mathbf{I}} \subset \overline{\mathcal{M}}_{\mathbf{J}}$.

\section{Lemma 3.5.}

(a) The type of a triple $(i, j, m)$ does not decrease in the Gromov limit on $\overline{\mathcal{M}}_{k+1}$. 
(b) A map $F: \mathcal{M}_{k+1} \rightarrow Z$ satisfies condition (b) in Definition 3.1 if and only if $\left.F\right|_{\mathcal{M}_{\mathbf{I}}}$ is a smooth function of the Type I-cross ratios for every stable decomposition $\mathbf{I}$.

(c) For stable decompositions $\mathbf{I} \neq \mathbf{J}$ such that $\mathbf{J}$ is a refinement of $\mathbf{I}$ there exists a cross ratio that is of Type I for $\mathbf{J}$ and Type II for $\mathbf{I}$.

(d) For stable decompositions $\mathbf{I} \neq \mathbf{J}$ such that $\mathbf{J}$ is not a refinement of $\mathbf{I}$ and $|\mathbf{I}| \geq 4$ there exists a cross ratio that is of Type I for $\mathbf{I}$ and Type II for $\mathbf{J}$.

Proof. (a) follows immediately from the definition Gromov compactness, see $[\mathbf{1 9}]$.

For (b) first suppose that $F$ satisfies condition (b) in Definition 3.1 and $\mathbf{I}$ is a stable decomposition with $|\mathbf{I}|=\ell+1 \geq 4$, so $\left.F\right|_{\mathcal{M}_{\mathbf{I}}}=F_{\mathbf{I}} \circ p_{\mathbf{I}}: \mathcal{M}_{\mathbf{I}} \rightarrow Z$ for a smooth map $F_{\mathbf{I}}: \mathcal{M}_{\ell+1} \rightarrow Z$. Denote points in $\mathcal{M}_{\ell+1}$ by $\left(z_{0}, \ldots, z_{\ell}\right)$. In view of Fact 1 above, $F_{\mathbf{I}}$ can be uniquely written as a smooth function of the cross ratios $\mathbf{w}_{0,1,2, j}$ with $3 \leq j \leq \ell$. By definition of $p_{\mathbf{I}}$ this means that $\left.F\right|_{\mathcal{M}_{\mathbf{I}}}$ is a smooth function of the Type I-cross ratios $\mathbf{w}_{i_{0}, i_{1}, i_{2}, i_{j}}$, where $i_{j}$ is the minimal element in $I_{j}$. The converse follows similarly.

For (c) choose indices $i \neq j$ that are equivalent for $\mathbf{I}$ and not equivalent for $\mathbf{J}$. Let $m$ be an index which is not equivalent to 0 or $i$ for $\mathbf{I}$, and hence also not for $J$. Then the cross ratio $w_{0, i, j, m}$ is of Type I for $\mathbf{J}$ and Type II for $\mathbf{I}$.

For $(\mathrm{d})$ choose a $J_{a}$ that is not contained in any $I_{b}$. Write $\mathbf{I}=\left(I_{0}, \ldots, I_{\ell}\right)$ with $\ell \geq 3$. Set $B:=\left\{b \in\{1, \ldots, \ell\} \mid J_{a} \cap I_{b} \neq \emptyset\right\}$ and denote by $\left\{J_{a} \cap I_{b}\right\}_{b \in B}$ the induced decomposition of $J_{a}$. Note that $|B| \geq 2$. Now we distinguish two cases. If $B \neq\{1, \ldots, \ell\}$ choose $d \in\{1, \ldots, \ell\} \backslash B$ and $b, c \in B$ with $b \neq c$, and choose $m \in I_{d}, i \in J_{a} \cap I_{b}$ and $j \in J_{a} \cap I_{c}$. If $B=\{1, \ldots, \ell\}$ choose pairwise distinct $b, c, d \in B$ such that $I_{d}$ is not contained in $J_{a}$ (this is possible since $\ell \geq 3$ and $|\mathbf{J}| \geq 3$ ), and choose $m \in I_{d} \backslash J_{a}, i \in J_{a} \cap I_{b}$ and $j \in J_{a} \cap I_{c}$. In both cases the cross ratio $w_{0, i, j, m}$ is of Type I for $\mathbf{I}$ and Type II for $\mathbf{J}$ (with $i \sim j)$.

Proof of Proposition 3.3. Fix a union $W$ of three disjoint disks around $0,1, \infty$ in $S^{2}$ and a cutoff function $\chi: S^{2} \rightarrow[0,1]$ with support in $W$ which equals 1 near $0,1, \infty$. In view of Fact 3 above, we can (and will) choose $W$ disjoint from the compact sets $w_{0, i, j, m}\left(\bar{U}_{\mathbf{I}}\right)$ for all Type I cross ratios $w_{0, i, j, m}$, $1 \leq i, j, m \leq|\mathbf{I}|$ associated to stable decompositions $\mathbf{I}$.

For $3 \leq \ell \leq k$ denote by $\phi_{\ell+1}: \mathcal{M}_{\ell+1} \hookrightarrow\left(S^{2}\right)^{\ell-2}$ the embedding given by the cross ratios $w_{0,1,2, j}$ with $3 \leq j \leq \ell$ (see Fact 1 above). In view of Fact 2 above, each stable decomposition $\mathbf{I}=\left(I_{0}, \ldots, I_{\ell}\right)$ induces a continuous map

$$
\phi_{\mathbf{I}}: \overline{\mathcal{M}}_{k+1} \longrightarrow\left(S^{2}\right)^{\ell-2}
$$


given by the cross ratios $w_{0, i_{1}, i_{2}, i_{j}}$ with $3 \leq j \leq \ell$. By construction, we have $\left.\phi_{\mathbf{I}}\right|_{\mathcal{M}_{\mathbf{I}}}=\phi_{\ell+1} \circ p_{\mathbf{I}}$ and $\phi_{\ell+1}\left(\bar{U}_{\mathbf{I}}\right) \subset\left(S^{2} \backslash W\right)^{\ell-2}$. The situation is summarized in the following diagram, where the first vertical arrow is the inclusion and the second one an embedding.

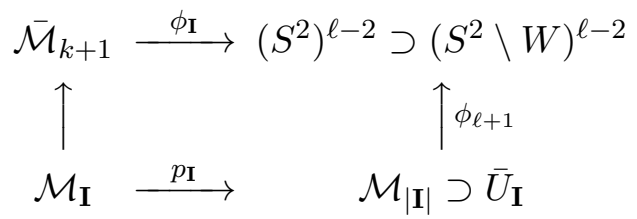

Composition yields a continuous linear embedding

$$
C_{0}^{\infty}\left(U_{\mathbf{I}}\right) \longrightarrow C_{0}^{0}\left(\overline{\mathcal{M}}_{k+1}\right), \quad \xi \longmapsto \xi \circ \phi_{\ell+1}^{-1} \circ \phi_{\mathbf{I}}
$$

satisfying $\left.\xi \circ \phi_{\ell+1}^{-1} \circ \phi_{\mathbf{I}}\right|_{\mathcal{M}_{\mathbf{I}}}=\xi \circ p_{\mathbf{I}}$. Define a continuous linear extension map $E_{\mathbf{I}}: C_{0}^{\infty}\left(U_{\mathbf{I}}, Z\right) \rightarrow C^{0}\left(\overline{\mathcal{M}}_{k+1}, Z\right)$ by

$$
E_{\mathbf{I}} \xi:=\left[\prod_{\substack{(i, j, m) \text { Type II } \\ \text { with respect to } \mathbf{I}}} \chi \circ w_{0, i, j, m}\right] \cdot \xi \circ \phi_{\ell+1}^{-1} \circ \phi_{\mathbf{I}}
$$

By construction, it satisfies

$$
\left.\left(E_{\mathbf{I}} \xi\right)\right|_{\mathcal{M}_{\mathbf{I}}}=\xi \circ \phi_{\ell+1}^{-1} \circ \phi_{\mathbf{I}}=\xi \circ p_{\mathbf{I}}
$$

so this restriction is continuous with respect to the $C^{\infty}$-topology via $p_{\mathbf{I}}$. Moreover, $E_{\mathbf{I}} \xi$ vanishes on the locus where some Type I-cross ratio with respect to $W$ takes values in $W$, so we could also write it as

$E_{\mathbf{I}} \xi=\left[\prod_{\substack{(i, j, m) \text { Type II } \\ \text { with respect to I }}} \chi \circ w_{0, i, j, m}\right] \cdot\left[\prod_{\begin{array}{c}(i, j, m) \text { Type I } \\ \text { with respect to I }\end{array}}(1-\chi) \circ w_{0, i, j, m}\right] \cdot \xi \circ \phi_{\ell+1}^{-1} \circ \phi_{\mathbf{I}}$

Consider now a stable decomposition $\mathbf{J} \neq \mathbf{I}$. Suppose first that $\mathbf{J}$ is not a refinement of $\mathbf{I}$. By Lemma 3.5(d), there exists a cross ratio $w_{0, i, j, k}$ that is of Type I for $\mathbf{I}$ and Type II for $\mathbf{J}$. It follows that $w_{0, i, j, k} \in\{0,1, \infty\}$ on $\mathcal{M}_{\mathbf{J}}$, so by the preceding discussion $E_{\mathbf{I}} \xi$ vanishes on $\mathcal{M}_{\mathbf{J}}$. Next suppose that $\mathbf{J}$ is a refinement of $\mathbf{I}$, i.e., $\mathcal{M}_{\mathbf{I}} \subset \overline{\mathcal{M}}_{\mathbf{J}}$. Note that $E_{\mathbf{I}} \xi$ depends only on cross ratios of Types I and II with respect to I. By Lemma 3.5(a), these cross ratios do 
not become Type III with respect to $\mathbf{J}$, so $\left.E_{\mathbf{I}} \xi\right|_{\mathcal{M}_{\mathbf{J}}}$ is a smooth function of Type I- and II-cross ratios with respect to J. But Type II-cross ratios are constant on $\mathcal{M}_{\mathbf{J}}$, so $\left.E_{\mathbf{I}} \xi\right|_{\mathcal{M}_{\mathbf{J}}}$ is a function of Type I-cross ratios and thus coherent by Lemma 3.5(b).

Next consider $\xi \in C_{0}^{\infty}\left(U_{\mathbf{I}}, Z\right)$ and $\eta \in C_{0}^{\infty}\left(U_{\mathbf{J}}, Z\right)$ with $\mathbf{I} \neq \mathbf{J}$. Suppose first that $\mathbf{J}$ is not a refinement of $\mathbf{I}$. By Lemma 3.5(d), there exists a cross ratio $w_{0, i, j, k}$ that is of Type I for $\mathbf{I}$ and Type II for $\mathbf{J}$. By construction, the support of $E_{\mathbf{J}} \eta$ is contained in the set $w_{0, i, j, m}^{-1}(W)$ and $E_{\mathbf{I}} \xi$ vanishes on this set, so $E_{\mathbf{I}} \xi$ and $E_{\mathbf{J}} \eta$ have disjoint support. Next suppose that $\mathbf{J}$ is a refinement of I. By Lemma 3.5(c), there exists a cross ratio $w_{0, i, j, m}$ that is of Type I for $\mathbf{J}$ and Type II for I. By construction, the support of $E_{\mathbf{I}} \xi$ is contained in the set $w_{0, i, j, m}^{-1}(W)$ and $E_{\mathbf{J}} \eta$ vanishes on this set, so again this shows that $E_{\mathbf{I}} \xi$ and $E_{\mathbf{J}} \eta$ have disjoint support.

Finally, the formula for the extension operator $E_{\mathbf{I}}$ in terms of cutoff functions shows that it preserves a star-shaped set $\mathbb{Z}^{*}$. This concludes the proof of Proposition 3.3.

Finally, we discuss the pullback of coherent maps under projection. For $I \subset\{1, \ldots, k\}$ with $|I| \geq 3$ denote by $\pi_{I}: \overline{\mathcal{M}}_{k+1} \rightarrow \overline{\mathcal{M}}_{|I|+1}$ the projection given by forgetting the marked points outside $I \cup\{0\}$ and stabilizing. For a stable decomposition $\mathbf{J}=\left(J_{0}, \ldots, J_{\ell}\right)$ of $\{0, \ldots, k\}$ denote by $\overline{\mathbf{J}}=\left(\bar{J}_{0}, \ldots, \bar{J}_{\bar{\ell}}\right)$ the induced decomposition of $I$ obtained by removing the points outside $I \cup\{0\}$. In general, $\bar{\ell}$ may be smaller than $\ell$ and $\overline{\mathbf{J}}$ need not be stable. We say that $\mathbf{J}$ is $I$-stable if $|\overline{\mathbf{J}}| \geq 3$, or equivalently, if $J_{i} \cap I \neq \emptyset$ for at least two $i \in\{1, \ldots, \ell\}$. Denote by $\mathcal{I}$ the collection of all $I$-stable decompositions. As before, $\pi: \overline{\mathcal{M}}_{k+1} \rightarrow \overline{\mathcal{M}}_{k}$ denotes the projection forgetting the marked point $z_{0}$ and stabilizing. We say that a $k$-labelled tree $T$ is $I$-stable if it is still stable after removing the marked points outside $I$.

Lemma 3.6 (Pullback under projection). Composition with $\pi_{I}$ induces a pullback map

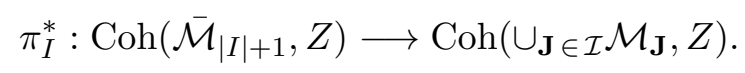

In particular, a coherent map $F: \overline{\mathcal{M}}_{|I|+1} \rightarrow Z$ induces a coherent map $\pi_{I}^{*} F$ : $\pi^{-1}\left(\mathcal{M}_{T}\right) \rightarrow Z$ for each $I$-stable $k$-labelled tree $T$.

Proof. Let $F \in \operatorname{Coh}\left(\overline{\mathcal{M}}_{|I|+1}, Z\right)$. Let $\mathbf{J}=\left(J_{0}, \ldots, J_{\ell}\right)$ be an $I$-stable decomposition of $\{0, \ldots, k\}$ and $\overline{\mathbf{J}}=\left(\bar{J}_{0}, \ldots, \bar{J}_{\bar{\ell}}\right)$ the induced decomposition of $I$ obtained by removing the points outside $I \cup\{0\}$. We claim that $\pi_{I}\left(\mathcal{M}_{\mathbf{J}}\right) \subset$ $\mathcal{M}_{\overline{\mathbf{J}}} \subset \overline{\mathcal{M}}_{I}$. Indeed, $I$-stability of $\mathbf{J}$ implies that for $\mathbf{z} \in \mathcal{M}_{\mathbf{J}}$ the component $\alpha_{0}$ is still stable after removing the points outside $I \cup\{0\}$ and therefore descends unchanged to $\pi_{I}(\mathbf{z})$. Hence $i, j \in I$ are equivalent in $\pi_{I}(\mathbf{z})$ iff they belong to the same $J_{m}$, i.e., to the same $\bar{J}_{m}$. 
Since $\left.F\right|_{\mathcal{M}_{\overline{\mathbf{J}}}}$ factors as $F_{\overline{\mathbf{J}}} \circ p_{\overline{\mathbf{J}}}$ for a smooth map $F_{\overline{\mathbf{J}}}: \mathcal{M}_{|\overline{\mathbf{J}}|} \rightarrow Z$, the following commutative diagram shows that $\left.\left(\pi_{I}^{*} F\right)\right|_{\mathcal{M}_{\mathbf{J}}}$ factors as $\left(F_{\overline{\mathbf{J}}} \circ \pi_{|\overline{\mathbf{J}}|}\right) \circ p_{\mathbf{J}}$ with the smooth map $F_{\overline{\mathbf{J}}} \circ \pi_{|\overline{\mathbf{J}}|}: \mathcal{M}_{|\mathbf{J}|} \rightarrow Z$.

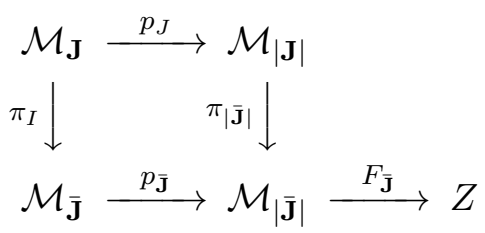

This proves condition (b) in Definition 3.1. Condition (a) also follows because $|\mathbf{J}|=3$ implies $|\bar{J}|=3$, so $F \circ \pi_{I} \equiv 0$ near such $\mathcal{M}_{\mathbf{J}}$. Finally, if $\mathbf{z} \in \mathcal{M}_{\mathbf{J}} \subset \mathcal{M}_{k+1}$ with $\pi(\mathbf{z}) \in \mathcal{M}_{T}$, then $I$-stability of $T$ implies that $|\overline{\mathbf{J}}| \geq 4$, in particular $\mathbf{J}$ is $I$-stable.

Almost complex structures. For a symplectic vector space $(V, \omega)$, denote by $\mathbf{J}(V, \omega)$ the space of $\omega$-tamed complex structures, i.e., endomorphisms $J: V \rightarrow V$ such that $J^{2}=-\mathbb{1}$ and $\omega(v, J v)>0$ for all tangent vectors $v$. The space $\mathbf{J}(V, \omega)$ is a manifold with tangent space

$$
T_{J_{0}} \mathbf{J}(V, \omega)=\left\{Y \in \operatorname{End}(V) \mid J_{0} Y J_{0}=Y\right\} .
$$

Suppose $V$ is equipped with a Euclidean metric $g$. Then $\operatorname{trace}\left(Y^{t} Y\right)$ defines a Riemannian metric on $\mathbf{J}(V, \omega)$. The exponential map of this metric defines embeddings

$$
\exp _{J}: T_{J} \mathbf{J}(V, \omega) \supset B(0, \rho(g, J)) \hookrightarrow \mathbf{J}(V, \omega)
$$

from the open ball of radius $\rho(g, J)>0$ which continuously depends on $g$ and $J$.

Following [9], for a vector bundle $E \rightarrow X$ over a closed manifold $X$ we denote the space of Floer's $C^{\varepsilon}$-sections in $E$ by

$$
C^{\varepsilon}(X, E):=\left\{s \in \Omega(X, E) \mid \sum_{i=1}^{\infty} \varepsilon_{i}\|s\|_{C^{i}}<\infty\right\} .
$$

Here $\varepsilon=\left(\varepsilon_{i}\right)_{i \in \mathbb{N}}$ is a fixed sequence of positive numbers and \|\|$_{C^{i}}$ is the $C^{i}$-norm with respect to some connection on $E$. It is shown in $[\mathbf{9}$, Lemma 5.1] that if the $\varepsilon_{i}$ converge sufficiently fast to zero, then $C^{\varepsilon}(X, E)$ is a Banach space consisting of smooth sections and containing sections with support in arbitrarily small sets in $X$.

Now let $(X, \omega)$ be a closed symplectic manifold. Fix a tamed almost complex structure $J_{0}$ on $(X, \omega)$, i.e., a smooth section in the bundle $\mathbf{J}(T X, \omega) \rightarrow$ $X$ with fibres $\mathbf{J}\left(T_{x} X, \omega_{x}\right)$. Define a Riemannian metric on $X$ via

$$
g(v, w):=\omega(v, J w)+\omega(w, J v) .
$$


Let $T_{J_{0}} \mathbf{J}(T X, \omega) \rightarrow X$ be the vector bundle with fibres $T_{J_{0}(x)} \mathbf{J}\left(T_{x} X, \omega_{x}\right)$ and set

$$
\begin{gathered}
T_{J_{0}} \mathcal{J}:=C^{\varepsilon}\left(X, T_{J_{0}} \mathbf{J}(T X, \omega)\right), \\
\mathcal{J}:=\mathcal{J}(X, \omega):=\exp _{J_{0}}(B),
\end{gathered}
$$

where $B:=\left\{Y \in T_{J_{0}} \mathcal{J} \mid Y(x) \in B\left(0, \rho\left(g(x), J_{0}(x)\right)\right)\right\}$. Thus $\mathcal{J}$ is the space of tamed almost complex structures of $(X, \omega)$ that are of class $C^{\varepsilon}$ over $J_{0}$ via $\exp _{J_{0}}$. We think of $\mathcal{J}$ as a Banach manifold with a single chart $\exp _{J_{0}}$.

Remark 3.7. We cannot replace the space $C^{\varepsilon}$ by $C^{\infty}$ because the latter one is not a Banach space and we need to apply the implicit function theorem below. However, we could replace $C^{\varepsilon}$ by $C^{N}$ for sufficiently large $N$ (which amounts to choosing $\varepsilon_{i}=0$ for $i>N$ ); then the moduli spaces of holomorphic spheres would not be smooth but of class $C^{N}$ as well, which suffices for the definition of Gromov-Witten invariants.

More generally, let $P$ be any manifold. A tamed almost complex structure on $(X, \omega)$ parametrized by $P$ is a smooth section in the pullback bundle $\mathbf{J}(T X, \omega) \rightarrow P \times X$. Fix $J_{0}$ as above. Let $T_{J_{0}} \mathbf{J}_{P}(T X, \omega) \rightarrow P \times X$ be the vector bundle with fibres $T_{J_{0}(p, x)} \mathbf{J}\left(T_{x} X, \omega_{x}\right)$ and set

$$
\begin{aligned}
T_{J_{0}} \mathcal{J}_{P} & :=C^{\varepsilon}\left(P \times X, T_{J_{0}} \mathbf{J}_{P}(T X, \omega)\right), \\
\mathcal{J}_{P} & :=\mathcal{J}_{P}(X, \omega):=\exp _{J_{0}}\left(B_{P}\right),
\end{aligned}
$$

where $B_{P}:=\left\{Y \in T_{J_{0}} \mathcal{J}_{P} \mid Y(p, x) \in B\left(0, \rho\left(g(x), J_{0}(x)\right)\right)\right\}$. Note that we may think of $J \in \mathcal{J}_{P}$ as a map $P \rightarrow \mathcal{J}$. For an open subset $U \subset P$, we denote by $T_{J} \mathcal{J}_{U} \subset T_{J} \mathcal{J}_{P}$ the subspace of those sections that have compact support in $U$. We will be interested in the spaces

$$
\mathcal{J}_{S^{2}}=\mathcal{J}_{S^{2}}(X, \omega) \quad \text { and } \quad \mathcal{J}_{\overline{\mathcal{M}}_{k+1}}=\mathcal{J}_{\overline{\mathcal{M}}_{k+1}}(X, \omega)
$$

parametrized by the Riemann sphere and the Deligne-Mumford space with $k+1$ marked points, respectively. To better understand the second case, recall that we have a holomorphic projection $\pi: \overline{\mathcal{M}}_{k+1} \rightarrow \overline{\mathcal{M}}_{k}$. Fix a stable curve $\mathbf{z}=\left(\left\{z_{\alpha \beta}\right\}_{\alpha E \beta},\left\{\mathbf{z}_{i}\right\}_{1 \leq i \leq k}\right) \in \overline{\mathcal{M}}_{k}$, modelled over the $k$-labelled tree $T$. Recall that $\pi^{-1}[\mathbf{z}]$ is naturally identified with the nodal Riemann surface $\Sigma_{\mathbf{z}}$ which is a union of $|T|$ copies $S_{\alpha}$ of $S^{2}$, glued together at the points $z_{\alpha \beta}$. Restriction of $J \in \mathcal{J}_{\overline{\mathcal{M}}_{k+1}}$ to $\pi^{-1}[\mathbf{z}]$ yields a continuous map

$$
J_{\mathbf{z}}: \Sigma_{\mathbf{z}} \longrightarrow \mathcal{J}
$$

which is smooth on each $S_{\alpha}$. We define the space $\mathcal{J}_{k+1}$ of coherent almost complex structures by

$$
\begin{aligned}
T_{J_{0}} \mathcal{J}_{k+1} & :=\operatorname{Coh}\left(\overline{\mathcal{M}}_{k+1}, T_{J_{0}} \mathcal{J}\right) \subset T_{J_{0}} \mathcal{J}_{\overline{\mathcal{M}}_{k+1}}, \\
\mathcal{J}_{k+1} & :=\exp _{J_{0}}\left(T_{J_{0}} \mathcal{J}_{k+1}\right) \subset \mathcal{J}_{\overline{\mathcal{M}}_{k+1}},
\end{aligned}
$$


where $\operatorname{Coh}\left(\overline{\mathcal{M}}_{k+1}, T_{J_{0}} \mathcal{J}\right) \subset T_{J_{0}} \mathcal{J}_{\overline{\mathcal{M}}_{k+1}}$ denotes the subspace of those maps $Y: \overline{\mathcal{M}}_{k+1} \rightarrow T_{J_{0}} \mathcal{J}$ satisfying the conditions of Definition 3.1.

Lemma 3.8. For $\mathbf{z} \in \overline{\mathcal{M}}_{k}$ modelled over the tree $T$ and $J \in \mathcal{J}_{k+1}$ the following holds.

(a) The restriction of $J_{\mathbf{z}}$ to each component $S_{\alpha}$ of $\Sigma_{\mathbf{z}}$ defines a smooth tamed almost complex structure $J_{\alpha} \in \mathcal{J}_{S_{\alpha}}$. Similarly, any $Y \in T_{J} \mathcal{J}_{k+1}$ restricts on $S_{\alpha}$ to a smooth section $\left.Y\right|_{S_{\alpha}} \in T_{J_{\alpha}} \mathcal{J}_{S_{\alpha}}$.

(b) Conversely, let $U_{\alpha} \subset S_{\alpha} \backslash \mathrm{SP}_{\alpha}$ be open subsets (for $\alpha \in T$ ), where $\mathrm{SP}_{\alpha}$ is the set of special points on $S_{\alpha}$ (except $\left.z_{0}\right)$. Then for any collection of sections $Y_{\alpha} \in T_{J_{\alpha}} \mathcal{J}_{U_{\alpha}}$ with compact support in $U_{\alpha}$, there exists a $Y \in T_{J} \mathcal{J}_{k+1}$ with $\left.Y\right|_{S_{\alpha}}=Y_{\alpha}$. Moreover, this extension is given by an injective continuous linear map

$$
\oplus_{\alpha} T_{J_{\alpha}} \mathcal{J}_{U_{\alpha}} \longrightarrow T_{J} \mathcal{J}_{k+1}
$$

Proof. Part (a) follows immediately from the fact (Lemma 2.4) that the $S_{\alpha}$ are submanifolds of $\overline{\mathcal{M}}_{k+1}$. The same fact also implies (b). Since $U_{\alpha} \times$ $X \subset \mathcal{M}_{n_{\alpha}+1} \times X$ is a submanifold, the section $Y_{\alpha} \in T_{J_{\alpha}} \mathcal{J}_{U_{\alpha}} \subset T_{J_{\alpha}} \mathcal{J}_{S_{\alpha}}$ of the vector bundle $T_{J} \mathbf{J}_{S_{\alpha}}(T X, \omega) \rightarrow S_{\alpha} \times X$ extends to a $C^{\varepsilon}$-section $\tilde{Y}_{\alpha}$ of the vector bundle $T_{J} \mathbf{J}_{\mathcal{M}_{n_{\alpha}+1}}(T X, \omega) \rightarrow \mathcal{M}_{n_{\alpha}+1} \times X$ with compact support. Now the construction proceeds exactly as in the proof of Proposition 3.3, choosing the cutoff function $\chi$ to be a $C^{\varepsilon}$-function.

\section{Holomorphic maps}

$J$-holomorphic maps. To a map $f: S^{2} \rightarrow X$ and a tamed almost complex structure $J \in \mathcal{J}_{S^{2}}$, we associate the $(0,1)$-form

$$
\bar{\partial}_{J} f:=\frac{1}{2}(d f+J(z, f) \circ d f \circ j)
$$

which at the point $z \in S^{2}$ is given by

$$
\bar{\partial}_{J} f(z):=\frac{1}{2}(d f(z)+J(z, f(z)) \circ d f(z) \circ j(z)) .
$$

We call $f J$-holomorphic if $\bar{\partial}_{J} f=0$. Then its energy is given by

$$
E(f):=\frac{1}{2} \int_{S^{2}}|d f|^{2} \mathrm{dvol}=\int_{S^{2}} f^{*} \omega .
$$

Fix an integer $m \geq 1$ and a real number $p>1$ such that $m p>2$ and define the Banach manifold

$$
\mathcal{B}:=\mathcal{B}^{m, p}:=W^{m, p}\left(S^{2}, X\right)
$$

of maps of Sobolev class $W^{m, p}$ (which are continuous because $m p>2$ ). Let

$$
\mathcal{E}:=\mathcal{E}^{m-1, p} \longrightarrow \mathcal{B}
$$


be the Banach bundle whose fibre at $f \in \mathcal{B}$ is given by $\mathcal{E}_{f}=W^{m-1, p}\left(S^{2}, \Omega^{0,1}\right.$ $\left.\left(f^{*} T X\right)\right)$. Then the Cauchy-Riemann operator defines a smooth section

$$
\bar{\partial}_{J}: \mathcal{B} \longrightarrow \mathcal{E}, \quad f \longmapsto \bar{\partial}_{J} f \text {. }
$$

More generally, the universal Cauchy-Riemann operator is the section

$$
\bar{\partial}: \mathcal{B} \times \mathcal{J}_{S^{2}} \longrightarrow \mathcal{E}, \quad(f, J) \longmapsto \bar{\partial}_{J} f .
$$

Now fix pairwise disjoint points $z_{1}, \ldots, z_{k} \in S^{2}$. Evaluation at these points defines a smooth map

$$
\mathrm{ev}^{k}: \mathcal{B} \longrightarrow X^{k}, \quad f \longmapsto\left(f\left(z_{1}\right), \ldots, f\left(z_{k}\right)\right) .
$$

Lemma 4.1. Let $k \in \mathbb{N}, f \in \mathcal{B}$ and $J \in \mathcal{J}_{S^{2}}$ satisfy $\bar{\partial}_{J} f=0$. If $f$ is nonconstant, then the linearization of

$$
\left(\bar{\partial}, \mathrm{ev}^{k}\right): \mathcal{B} \times \mathcal{J}_{S^{2}} \longrightarrow \mathcal{E} \times X^{k}
$$

at $(f, J)$ is surjective. More precisely, for any nonempty open subset $U \subset S^{2} \backslash$ $\left\{z_{1}, \ldots, z_{k}\right\}$, the restriction of the linearization to the subspace

$$
T_{f} \mathcal{B} \oplus T_{J} \mathcal{J}_{U} \subset T_{f} \mathcal{B} \oplus T_{J} \mathcal{J}_{S^{2}}
$$

of sections with support in $U$ is surjective.

Proof. The proof is very similar to that of [19, Proposition 3.4.2], so we only sketch the argument. The linearization of $\left(\bar{\partial}, \mathrm{ev}^{k}\right)$ at $(f, J)$, restricted to the subspace $T_{f} \mathcal{B}^{m, p} \oplus T_{J} \mathcal{J}_{U}$, is the linear operator

$$
\begin{gathered}
L: T_{f} \mathcal{B}^{m, p} \oplus T_{J} \mathcal{J}_{U} \longrightarrow \mathcal{E}_{f} \oplus \bigoplus_{i=1}^{k} T_{f\left(z_{i}\right)} X, \\
(\xi, Y) \longmapsto\left(D_{f} \xi+\frac{1}{2} Y(z, f) \circ d f \circ j, \xi\left(z_{1}\right), \ldots, \xi\left(z_{k}\right)\right),
\end{gathered}
$$

where $D_{f}: T_{f} \mathcal{B}^{m, p} \rightarrow \mathcal{E}_{f}$ is the linearization of $\bar{\partial}_{J}$ at $f$. We will prove surjectivity of the operator

$$
\begin{gathered}
L_{0}: B_{0}^{m, p} \oplus T_{J} \mathcal{J}_{U} \longrightarrow \mathcal{E}_{f}, \\
(\xi, Y) \longmapsto D_{f} \xi+\frac{1}{2} Y(z, f) \circ d f \circ j,
\end{gathered}
$$

where $B_{0}^{m, p}:=\left\{\xi \in T_{f} \mathcal{B}^{m, p} \mid \xi\left(z_{1}\right)=\cdots=\xi\left(z_{k}\right)=0\right\}$. Surjectivity of $L_{0}$ easily implies surjectivity of $L$ : Given $\left(\eta, v_{1}, \ldots, v_{k}\right) \in \mathcal{E}_{f} \oplus \bigoplus_{i=1}^{k} T_{f\left(z_{i}\right)} X$, pick a $\xi_{1} \in T_{f} \mathcal{B}^{m, p}$ with $\xi_{1}\left(z_{i}\right)=v_{i}$ for all $i$ and find $\left(\xi_{2}, Y\right) \in B_{0}^{m, p} \oplus T_{J} \mathcal{J}_{U}$ with $L_{0}(\xi, Y)=\eta-D_{f} \xi_{1}$, hence $L\left(\xi_{1}+\xi_{2}, Y\right)=\left(\eta, v_{1}, \ldots, v_{k}\right)$.

To prove surjectivity of $L_{0}$, first consider the case $m=1$. Since $D_{f}$ is a Fredholm operator, the image of $L_{0}$ is closed. So it suffices to show that every element $\eta \in \mathcal{E}_{f}^{*}$ orthogonal to $\operatorname{im}\left(L_{0}\right)$ is zero. Note that $\mathcal{E}_{f}$ is a space of $L^{p_{-}}$ sections, so its dual space $\mathcal{E}_{f}^{*}$ can be identified with the appropriate space of 
$L^{q}$-sections with $1 / p+1 / q=1$ via the $L^{2}$ inner product. Thus orthogonality to $\operatorname{im}\left(L_{0}\right)$ amounts to the equations

$$
\begin{gathered}
\left\langle D_{f} \xi, \eta\right\rangle_{L^{2}}=0, \\
\langle Y(z, f) \circ d f \circ j, \eta\rangle_{L^{2}}=0
\end{gathered}
$$

for all $(\xi, Y) \in B_{0}^{1, p} \oplus T_{J} \mathcal{J}_{U}$. By [19, Lemma 3.4.7], the first equation implies that $\eta$ is smooth on $S^{2} \backslash\left\{z_{0}, \ldots, z_{k}\right\}$ and satisfies on this set the Cauchy-Riemann type equation $D_{f}^{*} \eta=0$. Since $f$ is nonconstant, the set $U^{*}:=\{z \in U \mid d f(z) \neq 0\}$ is open and dense in $U$. Suppose that $\eta(z) \neq 0$ for some $z \in U^{*}$. Then by [19, Lemma 3.2.2] and cutting off near $z$, we find a $Y \in T_{J} \mathcal{J}_{U}$ such that $\left\langle Y\left(z^{\prime}, f\right) \circ d f\left(z^{\prime}\right) \circ j, \eta\left(z^{\prime}\right)\right\rangle \geq 0$ for all $z^{\prime}$ and $>0$ at $z^{\prime}=z$, in contradiction to the second equation. (Here we have used the fact that $Y$ may depend on points in $U$, which allows us to cut off $Y$ near $z$.) It follows that $\eta(z)=0$ for all $z \in U^{*}$, and hence by unique continuation (see [19, Lemma 3.4.7]) $\eta \equiv 0$. This proves surjectivity of $L_{0}$ in the case $m=1$. The case of general $m \geq 1$ follows from the $m=1$ case by elliptic regularity. Given $\eta \in \mathcal{E}_{f}^{m-1, p}$, by the $m=1$ case we find $(\xi, Y) \in B_{0}^{1, p} \oplus T_{J} \mathcal{J}_{U}$ with $L_{0}(\xi, Y)=\eta$, and elliptic regularity (see [19, Theorem C.2.3]) yields $\xi \in B_{0}^{m, p}$. This concludes the proof of Lemma 4.1 .

For constant $f$ we have a similar result with $k=1$.

Lemma 4.2. If $f \in \mathcal{B}$ is constant and $J \in \mathcal{J}_{S^{2}}$, then the linearization of

$$
\left(\bar{\partial}_{J}, \mathrm{ev}^{1}\right): \mathcal{B} \longrightarrow \mathcal{E} \times X
$$

at $f$ is surjective.

Proof. Let $f \equiv x \in X$ and identify $T_{x} X$ with $\mathbb{R}^{2 n}$. Then the linearization of $\bar{\partial}_{J}$ at $f$ is the Cauchy-Riemann operator

$$
L: W^{m, p}\left(S^{2}, \mathbb{R}^{2 n}\right) \longrightarrow W^{m-1, p}\left(\Omega^{0,1}\left(S^{2}, \mathbb{R}^{2 n}\right)\right), \quad \xi \longmapsto \bar{\partial}_{J} \xi
$$

for a family of linear complex structures $J(z)$ on $\mathbb{R}^{2 n}, z \in S^{2}$. Elements in the kernel of $L$ have zero energy (since they are homologically trivial) and are therefore constant. Since $L$ has index $2 n$, it follows that $L$ is surjective and its kernel consists of the constant functions. Hence the linearized evaluation map $\xi \longmapsto \xi\left(z_{1}\right)$ is surjective on the kernel of $L$ and the result follows.

Lemmas 4.1 and 4.2 immediately imply

Proposition 4.3. The universal solution space

$$
\tilde{\mathcal{M}}\left(\mathcal{J}_{S^{2}}\right):=\left\{(f, J) \in \mathcal{B} \times \mathcal{J}_{S^{2}} \mid \bar{\partial}_{J} f=0\right\}
$$

is a Banach manifold and the 1-point evaluation map

$$
\mathrm{ev}^{1}: \tilde{\mathcal{M}}\left(\mathcal{J}_{S^{2}}\right) \longrightarrow X
$$


is a submersion. Moreover, for any $k \in \mathbb{N}$ the $k$-point evaluation map

$$
\mathrm{ev}^{k}: \tilde{\mathcal{M}}^{\mathrm{nc}}\left(\mathcal{J}_{S^{2}}\right) \longrightarrow X^{k}
$$

is a submersion on the open component $\tilde{\mathcal{M}}^{\mathrm{nc}}\left(\mathcal{J}_{S^{2}}\right) \subset \tilde{\mathcal{M}}\left(\mathcal{J}_{S^{2}}\right)$ of pairs $(f, J)$ with $f$ nonconstant.

For a homology class $A \in H_{2}(X ; \mathbb{Z}), J \in \mathcal{J}_{S^{2}}$ and a smooth submanifold $Z \subset X^{k}$ define

$$
\begin{gathered}
\tilde{\mathcal{M}}(A, J):=\left\{f: S^{2} \longrightarrow X \mid \bar{\partial}_{J} f=0,[f]=A\right\}, \\
\tilde{\mathcal{M}}(A, J ; Z):=\left\{f \in \tilde{\mathcal{M}}(A, J) \mid\left(f\left(z_{1}\right), \ldots, f\left(z_{k}\right)\right) \in Z\right\} .
\end{gathered}
$$

Taking regular values of the projection $(f, J) \mapsto J$, we obtain

Corollary 4.4. For every smooth submanifold $Z \subset X$ there exists a Baire set $\mathcal{J}_{S^{2}}^{\text {reg }}(Z) \subset \mathcal{J}_{S^{2}}$ such that for every homology class $A \in H_{2}(X ; \mathbb{Z})$ and every $J \in \mathcal{J}_{S^{2}}^{\mathrm{reg}}(Z)$ the solution space

$$
\tilde{\mathcal{M}}(A, J ; Z)=\left\{f \in \tilde{\mathcal{M}}(A, J) \mid f\left(z_{1}\right) \in Z\right\}
$$

is a smooth manifold of dimension

$$
\operatorname{dim} \tilde{\mathcal{M}}(A, J ; Z)=2 n+2 c_{1}(A)-\operatorname{codim}_{\mathbb{R}} Z .
$$

More generally, for every smooth submanifold $Z \subset X^{k}$ there exists a Baire set $\mathcal{J}_{S^{2}}^{\mathrm{reg}}(Z) \subset \mathcal{J}_{S^{2}}$ such that for every nonzero homology class $0 \neq A \in H_{2}(X ; \mathbb{Z})$ and every $J \in \mathcal{J}_{S^{2}}^{\mathrm{reg}}(Z)$ the solution space

$$
\tilde{\mathcal{M}}(A, J ; Z)=\left\{f \in \tilde{\mathcal{M}}(A, J) \mid\left(f\left(z_{1}\right), \ldots, f\left(z_{k}\right)\right) \in Z\right\}
$$

is a smooth manifold of dimension

$$
\operatorname{dim} \tilde{\mathcal{M}}(A, J ; Z)=2 n+2 c_{1}(A)-\operatorname{codim}_{\mathbb{R}} Z .
$$

\section{Nodal maps}

Throughout this section, we fix a symplectic manifold $(X, \omega)$ of dimension $2 n$, not necessarily closed.

Weighted trees. A weighted $k$-labelled tree $\left(T,\left\{A_{\alpha}\right\}\right)$ is a $k$-labelled tree $T$ with a collection of homology classes $A_{\alpha} \in H_{2}(X ; \mathbb{Z}), \alpha \in T$. We call $\left(T,\left\{A_{\alpha}\right\}\right)$ weighted stable if each component $\alpha \in T$ with $A_{\alpha}=0$ carries at least three special points. Note that stability implies weighted stability but not vice versa. We call a component $\alpha$ with $A_{\alpha}=0$ a ghost component, and a maximal subtree consisting of ghost components a ghost tree. The reduced index set of $\left(T,\left\{A_{\alpha}\right\}\right)$ is the subset $R \subset\{1, \ldots, k\}$ containing all marked points on components with $A_{\alpha} \neq 0$, and the unique marked point $z_{i}$ with the maximal index $i$ on each ghost tree. 
Stable maps. Fix a $k$-labelled tree $T=(T, E, \Lambda)$ (not necessarily stable). Let

$$
\mathcal{J}_{T}:=\prod_{\alpha \in T} \mathcal{J}_{S_{\alpha}}
$$

be the space of collections $\mathbf{J}=\left\{J_{\alpha}\right\}_{\alpha \in T}$ of tamed almost complex structures $J_{\alpha} \in \mathcal{J}_{S_{\alpha}}$ parametrized by the spheres $S_{\alpha}$. Let $\mathbf{z}=\left(\left\{z_{\alpha \beta}\right\}_{\alpha E \beta},\left\{\mathbf{z}_{i}\right\}_{1 \leq i \leq k}\right)$ be a nodal curve modelled over $T$ and $\Sigma_{\mathbf{z}}=\cup_{\alpha \in T} S_{\alpha}$ the associated nodal Riemann surface. A continuous map $\mathbf{f}: \Sigma_{\mathbf{z}} \rightarrow X$ is a collection $\left\{f_{\alpha}\right\}_{\alpha \in T}$ of continuous maps $f_{\alpha}: S_{\alpha} \rightarrow X$ such that $f_{\alpha}\left(z_{\alpha \beta}\right)=f_{\beta}\left(z_{\beta \alpha}\right)$ whenever $\alpha E \beta$. Note that each such map f induces homology classes $A_{\alpha}=\left[f_{\alpha}\right] \in H_{2}(X ; \mathbb{Z})$ and thus weights on the tree $T$. To $\mathbf{J} \in \mathcal{J}_{T}$ and $\mathbf{f}: \Sigma_{\mathbf{z}} \rightarrow X$ we associate the Cauchy-Riemann operator

$$
\bar{\partial}_{\mathbf{J}} \mathbf{f}:=\frac{1}{2}(d \mathbf{f}+\mathbf{J}(z, \mathbf{f}) \circ d \mathbf{f} \circ j)
$$

which at the point $z \in S_{\alpha}$ is given by

$$
\bar{\partial}_{J_{\alpha}} f_{\alpha}(z):=\frac{1}{2}\left(d f_{\alpha}(z)+J_{\alpha}\left(z, f_{\alpha}(z)\right) \circ d f_{\alpha}(z) \circ j(z)\right) .
$$

A continuous map $\mathbf{f}$ is called $\mathbf{J}$-holomorphic if $\bar{\partial}_{\mathbf{J}} \mathbf{f}=0$ (hence each $f_{\alpha}$ is smooth by elliptic regularity). A pair ( $\mathbf{z}, \mathbf{f})$ consisting of a nodal curve $\mathbf{z}$ and a $\mathbf{J}$-holomorphic map $\mathbf{f}: \Sigma_{\mathbf{z}} \rightarrow X$ is called a nodal $\mathbf{J}$-holomorphic map of genus zero with $k$ marked points, or simply a nodal map. Note that a nodal map $(\mathbf{z}, \mathbf{f})$ represents a homology class

$$
[\mathbf{f}]:=\sum_{\alpha \in T}\left[f_{\alpha}\right] \in H_{2}(X ; \mathbb{Z})
$$

and carries the energy

$$
E(\mathbf{f}):=\sum_{\alpha \in T} E\left(f_{\alpha}\right) .
$$

We say that a nodal map $(\mathbf{z}, \mathbf{f})$ is modelled over the weighted tree $\left(T,\left\{A_{\alpha}\right\}\right)$ if $\mathbf{z}$ is modelled over $T$ and $\left[f_{\alpha}\right]=A_{\alpha}$ for all $\alpha \in T$. A nodal map $(\mathbf{z}, \mathbf{f})$ is called stable if the underlying weighted tree $\left(T,\left\{A_{\alpha}=\left[f_{\alpha}\right]\right\}\right)$ is weighted stable, i.e., if each component $\alpha \in T$ for which $f_{\alpha}$ is constant carries at least three special points.

For a weighted tree $\left(T,\left\{A_{\alpha}\right\}\right)$ and $\mathbf{J} \in \mathcal{J}_{T}$ let

$$
\tilde{\mathcal{M}}_{T}\left(\left\{A_{\alpha}\right\}, \mathbf{J}\right)
$$

be the space of all nodal maps $(\mathbf{z}, \mathbf{f})$ modelled over $\left(T,\left\{A_{\alpha}\right\}\right)$. The space of stable maps modelled on the tree $T$ representing the class $A$ is the disjoint union

$$
\tilde{\mathcal{M}}_{T}(A, \mathbf{J}):=\coprod_{\sum A_{\alpha}=A} \tilde{\mathcal{M}}_{T}\left(\left\{A_{\alpha}\right\}, \mathbf{J}\right),
$$

where the union is taken over all decompositions $\left\{A_{\alpha}\right\}$ of $A$ such that $\left(T,\left\{A_{\alpha}\right\}\right)$ is weighted stable. 
Domain-stable maps. Fix an integer $k \geq 3$ and consider the space $\mathcal{J}_{k+1}$ of coherent tamed almost complex structures on $(X, \omega)$ parametrized by the Deligne-Mumford space $\overline{\mathcal{M}}_{k+1}$. Fix a stable curve $\mathbf{z}=\left(\left\{z_{\alpha \beta}\right\}_{\alpha E \beta},\left\{\mathbf{z}_{i}\right\}_{1 \leq i \leq k}\right)$, modelled over the $k$-labelled tree $T=(T, E, \Lambda)$. Restriction of $J \in \mathcal{J}_{k+1}$ to $\pi^{-1}[\mathbf{z}] \cong \Sigma_{\mathbf{z}}=\cup_{\alpha \in T} S_{\alpha}$ yields a map

$$
J_{\mathbf{z}}: \Sigma_{\mathbf{z}} \longrightarrow \mathcal{J} \text {. }
$$

By Lemma 3.8, the restriction of $J_{\mathbf{z}}$ to each component $S_{\alpha}$ of $\Sigma_{\mathbf{z}}$ is smooth, so $J_{\mathbf{z}}$ yields an element in $\mathcal{J}_{T}=\prod_{\alpha \in T} \mathcal{J}_{S_{\alpha}}$. As above, to a map $\mathbf{f}=\left\{f_{\alpha}\right\}_{\alpha \in T}$ : $\Sigma_{\mathbf{z}} \rightarrow X$ and $J \in \mathcal{J}_{k+1}$ we associate the Cauchy-Riemann operator

$$
\bar{\partial}_{J_{\mathbf{z}}} \mathbf{f}:=\frac{1}{2}\left(d \mathbf{f}+J_{\mathbf{z}}(z, \mathbf{f}) \circ d \mathbf{f} \circ j\right)
$$

which at the point $z \in S_{\alpha}$ is given by

$$
\bar{\partial}_{J_{\mathbf{z}}} f_{\alpha}(z):=\frac{1}{2}\left(d f_{\alpha}(z)+J_{\mathbf{z}}\left(z, f_{\alpha}(z)\right) \circ d f_{\alpha}(z) \circ j(z)\right) .
$$

If $\mathbf{z}$ is a stable curve and $\mathbf{f}$ is $J_{\mathbf{z}}$-holomorphic for $J \in \mathcal{J}_{k+1}$, we call the pair $(\mathbf{z}, \mathbf{f})$ a domain-stable $J$-holomorphic map of genus zero with $k$ marked points, or simply a domain-stable map. Note that every domain-stable map is stable, but domain-stability is more restrictive because it requires the underlying curve to be stable.

More generally, let $\mathbf{z}$ be a nodal curve with $k$ marked points, not necessarily stable. By Lemma 2.5, stabilization yields a holomorphic map st $: \Sigma_{\mathbf{z}} \rightarrow \Sigma_{\text {st( }(\mathbf{z})}$. So $J \in \mathcal{J}_{k+1}$ yields a map

$$
J_{\mathbf{z}}^{\text {st }}: \Sigma_{\mathbf{z}} \longrightarrow \mathcal{J}, \quad J_{\mathbf{z}}^{\text {st }}(z):=J_{\text {st }(\mathbf{z})}(\operatorname{st}(z))
$$

whose restriction to each sphere $S_{\alpha}$ is smooth. Note that $J_{\mathbf{z}}^{\text {st }}$ is constant on the spheres $S_{\alpha}$ with $\alpha$ in the collection of subtrees $T^{\prime} \subset T$ from Lemma 2.5. This construction allows us to define the Cauchy-Riemann operator $\bar{\partial}_{J_{\mathbf{z}}^{\text {st }}} \mathbf{f}$ as above and speak about nodal $J$-holomorphic maps $(\mathbf{z}, \mathbf{f})$ for $J \in \mathcal{J}_{k+1}$. As above, let

$$
\tilde{\mathcal{M}}_{T}(A, J):=\coprod_{\sum A_{\alpha}=A} \tilde{\mathcal{M}}_{T}\left(\left\{A_{\alpha}\right\}, J\right),
$$

be the space of stable $J$-holomorphic maps modelled on the (not necessarily stable) $k$-labelled tree $T$ for $J \in \mathcal{J}_{k+1}$.

Moduli spaces. Let $J \in \mathcal{J}_{k+1}$. An isomorphism between two nodal $J$-holomorphic maps $(\mathbf{z}, \mathbf{f})$ and $(\tilde{\mathbf{z}}, \tilde{\mathbf{f}})$ is an isomorphism $\left(\tau,\left\{\phi_{\alpha}\right\}_{\alpha \in T}\right)$ between the nodal curves $\mathbf{z}$ and $\tilde{\mathbf{z}}$ such that $\tilde{f}_{\tau(\alpha)} \circ \phi_{\alpha}=f_{\alpha}$ for all $\alpha \in T$. The following lemma shows that $J$-holomorphicity is preserved under isomorphisms.

Lemma 5.1. Let $\mathbf{z}, \tilde{\mathbf{z}}$ be nodal curves with $k$ marked points and $\mathbf{f}: \Sigma_{\mathbf{z}} \rightarrow X$, $\tilde{\mathbf{f}}: \Sigma_{\tilde{\mathbf{z}}} \rightarrow X$ be continuous maps. Let $\phi=\left(\tau,\left\{\phi_{\alpha}\right\}_{\alpha \in T}\right): \mathbf{z} \rightarrow \tilde{\mathbf{z}}$ be an 
isomorphism between the nodal curves such that $\tilde{f}_{\tau(\alpha)} \circ \phi_{\alpha}=f_{\alpha}$ for all $\alpha \in T$. Let $J \in \mathcal{J}_{k+1}$. Then $\mathbf{f}$ is J-holomorphic if and only if $\tilde{\mathbf{f}}$ is.

Proof. As mentioned in the definition of morphisms between nodal curves we will denote the induced map between the nodal curves by the same letter. By definition of $\mathcal{J}_{k+1}, J$ is invariant under isomorphisms $\psi$ in the sense that $J_{\psi(\mathbf{w})}(\psi(w))=J_{\mathbf{w}}(w)$ for $\mathbf{w} \in \tilde{\mathcal{M}}_{k}$ and $w \in \Sigma_{\mathbf{w}}$. By Lemma 2.5, the isomorphism $\phi: \mathbf{z} \rightarrow \tilde{\mathbf{z}}$ induces an isomorphism $\phi^{\text {st }}: \operatorname{st}(\mathbf{z}) \rightarrow \operatorname{st}(\tilde{\mathbf{z}})$ between the stabilized curves such that st $\circ \phi=\phi^{\text {st }} \circ$ st. It follows that

$$
\begin{aligned}
J_{\phi(\mathbf{z})}^{\mathrm{st}}(\phi(z)) & =J_{\mathrm{st}(\phi(\mathbf{z}))}(\operatorname{st}(\phi(z)))=J_{\phi^{\mathrm{st}}(\operatorname{st}(\mathbf{z}))}\left(\phi^{\mathrm{st}}(\operatorname{st}(z))\right) \\
& =J_{\mathrm{st}(\mathbf{z})}(\operatorname{st}(z))=J_{\mathbf{z}}^{\mathrm{st}}(z) .
\end{aligned}
$$

Together with holomorphicity of $\phi_{\alpha}$, this implies

$$
\bar{\partial} \tilde{\mathbf{f}}=\bar{\partial}_{J_{\phi(\mathbf{z})}^{\mathrm{st}}}\left(f_{\alpha} \circ \phi_{\alpha}^{-1}\right)(\phi(z))=\bar{\partial}_{J_{\mathbf{z}}^{\text {st }}} f_{\alpha}(z) \circ d \phi_{\alpha}(z)^{-1}
$$

for every $\alpha \in T$ and $z \in S_{\alpha}$, and the lemma follows.

It follows that for every tree $T$ and homology class $A$ the group $G_{T}$ of isomorphisms fixing $T$ (but not necessarily the identity on $T$ ) acts on the space $\tilde{\mathcal{M}}_{T}(A, J)$ of stable maps. The action of $G_{T}$ is proper (see [19, Section 6.1]; the argument is similar to the remark in Section 2 above), and due to the stability condition it has only finite isotropy groups (see [19]). Denote its quotient space by

$$
\mathcal{M}_{T}(A, J):=\tilde{\mathcal{M}}_{T}(A, J) / G_{T} .
$$

The moduli space of stable maps (of genus zero with $k$ marked points representing the class $A$ ) is the disjoint union

$$
\overline{\mathcal{M}}_{k}(A, J)=\bigcup_{T} \mathcal{M}_{T}(A, J)
$$

over the isomorphism classes of $k$-labelled trees. It carries a natural topology, the Gromov topology defined in [19], which makes it into a metrizable space. We call the $\mathcal{M}_{T}(A, J)$ the strata of $\overline{\mathcal{M}}_{k}(A, J)$. In particular, we have the top stratum $\mathcal{M}_{k}(A, J):=\mathcal{M}_{T_{k}}(A, J)$ of stable maps modelled over the $k$-labelled tree $T_{k}$ with one vertex.

The moduli space of domain-stable maps is the subset

$$
\overline{\mathcal{M}}_{k}^{\mathrm{ds}}(A, J):=\bigcup_{T \text { stable }} \mathcal{M}_{T}(A, J) \subset \overline{\mathcal{M}}_{k}(A, J)
$$

of those nodal maps whose underlying curve is stable. Note that if the tree $T$ is stable, then the action of $G_{T}$ on $\tilde{\mathcal{M}}_{T}(A, J)$ is free and covers the trivial 
action on $T$ (see [19]). So in this case the quotient space has a natural decomposition

$$
\mathcal{M}_{T}(A, J)=\coprod_{\sum A_{\alpha}=A} \mathcal{M}_{T}\left(\left\{A_{\alpha}\right\}, J\right), \quad \mathcal{M}_{T}\left(\left\{A_{\alpha}\right\}, J\right)=\tilde{\mathcal{M}}_{T}\left(\left\{A_{\alpha}\right\}, J\right) / G_{T} .
$$

(If $T$ is unstable the corresponding decomposition need not descend to the quotient because isomorphisms may interchange vertices of $T$.)

Compactness. We now state Gromov's compactness theorem in our context. It is proved in [19] for almost complex structures $J$ not depending on points in $\overline{\mathcal{M}}_{k+1}$, but the proof immediately carries over to our situation.

Theorem 5.2. Let $J \in \mathcal{J}_{k+1}$. Let $\left(\mathbf{z}^{\nu}, \mathbf{f}^{\nu}\right)$ be a sequence of stable $J$-holomorphic maps (of genus zero with $k$ marked points) with uniformly bounded energy $E\left(\mathbf{f}^{\nu}\right) \leq C$. Then a subsequence converges in the Gromov topology to a stable $J$-holomorphic map $(\mathbf{z}, \mathbf{f})$ (of genus zero with $k$ marked points).

For the precise definition of Gromov convergence see [19]. It implies, in particular, that after passing to a subsequence the following properties hold:

(1) The $\left(\mathbf{z}^{\nu}, \mathbf{f}^{\nu}\right)$ all belong to the same moduli space $\mathcal{M}_{\tilde{T}}\left(\left\{A_{\tilde{\alpha}}\right\}, J\right)$ for some stable weighted tree $\left(\tilde{T},\left\{A_{\tilde{\alpha}}\right\}\right)$.

(2) The limit map belongs to some moduli space $\mathcal{M}_{T}\left(\left\{A_{\alpha}\right\}, J\right)$ and there exists a surjective tree homomorphism $\tau: T \rightarrow \tilde{T}$ such that

$$
\tau\left(\alpha_{i}\right)=\tilde{\alpha}_{i}, \quad \sum_{\alpha \in \tau^{-1}(\tilde{\alpha})} A_{\alpha}=A_{\tilde{\alpha}}
$$

for $i=1, \ldots, k$ and $\tilde{\alpha} \in \tilde{T}$.

(3) The stabilizations $\operatorname{st}\left(\mathbf{z}^{\nu}\right)$ converge to the stabilization $\operatorname{st}(\mathbf{z})$ and $\mathbf{f}$ is $J_{\text {st }(\mathbf{z})}$-holomorphic (cf. [19, Theorem 5.6.6]).

(4) After suitable reparametrizations, the $\mathbf{f}^{\nu}$ converge uniformly to $\mathbf{f}$.

Remark 5.3. By Theorem 5.2 the moduli space $\overline{\mathcal{M}}_{k}(A, J)$ of stable maps is compact. We wish to emphasize, however, that the subset $\overline{\mathcal{M}}_{k}^{\mathrm{ds}}(A, J) \subset$ $\overline{\mathcal{M}}_{k}(A, J)$ of domain-stable maps is in general not closed in the Gromov topology. The underlying nodal curve of the Gromov limit of a sequence of domain-stable maps need not be stable. However, in Section 9 we will encounter a situation in which $\overline{\mathcal{M}}_{k}^{\mathrm{ds}}(A, J)$ (with additional constraints) is closed in $\overline{\mathcal{M}}_{k}(A, J)$.

Transversality. The aim of this section is to show that for a stable tree $T$, the space $\mathcal{M}_{T}(A, J)$ is a manifold for generic $J \in \mathcal{J}_{k+1}$, as well as various refinements of this result. For this, we need some more notation.

Fix a nodal curve $\mathbf{z}=\left(\left\{z_{\alpha \beta}\right\}_{\alpha E \beta},\left\{\mathbf{z}_{i}\right\}_{1 \leq i \leq k}\right)$ with $k$ marked points, modelled over the $k$-labelled tree $T=(T, E, \Lambda)$. Fix $m p>2$ and homology classes 
$A_{\alpha}, \alpha \in T$, and set

$$
\mathcal{B}\left(\left\{A_{\alpha}\right\}\right):=\mathcal{B}^{m, p}\left(\left\{A_{\alpha}\right\}\right):=\prod_{\alpha \in T} \mathcal{B}^{m, p}\left(A_{\alpha}\right),
$$

where $\mathcal{B}^{m, p}\left(A_{\alpha}\right)$ denotes the space of maps $S^{2} \rightarrow X$ of Sobolev class $W^{m, p}$ representing the homology class $A_{\alpha}$. We write elements in $\mathcal{B}\left(\left\{A_{\alpha}\right\}\right)$ as $\mathbf{f}=\left(f_{\alpha}\right)_{\alpha \in T}$ with $f_{\alpha}: S_{\alpha} \rightarrow X$. Let $X^{E}$ be the product indexed by pairs $\alpha \beta$ such that $\alpha E \beta$, and let the edge diagonal $\Delta^{E} \subset X^{E}$ be defined by $x_{\alpha \beta}=x_{\beta \alpha}$ for $\alpha E \beta$. Evaluation at the $z_{\alpha \beta}$ defines the edge evaluation map

$$
\mathrm{ev}^{E}: \mathcal{B}\left(\left\{A_{\alpha}\right\}\right) \longrightarrow X^{E} \text {. }
$$

Note that $\mathbf{f} \in \mathcal{B}\left(\left\{A_{\alpha}\right\}\right)$ induces a continuous map $\Sigma_{\mathbf{z}} \rightarrow X$ iff $\mathrm{ev}^{E}(\mathbf{f}) \in \Delta^{E}$. For $\mathbf{J}=\left(J_{\alpha}\right) \in \mathcal{J}_{T}$, the Cauchy-Riemann operators $f_{\alpha} \mapsto \bar{\partial}_{J_{\alpha}} f_{\alpha}$ define a smooth section

$$
\bar{\partial}_{\mathbf{J}}: \mathcal{B}\left(\left\{A_{\alpha}\right\}\right) \longrightarrow \mathcal{E}\left(\left\{A_{\alpha}\right\}\right)
$$

in the bundle

$$
\mathcal{E}\left(\left\{A_{\alpha}\right\}\right):=\mathcal{E}^{m-1, p}\left(\left\{A_{\alpha}\right\}\right):=\prod_{\alpha \in T} \mathcal{E}^{m-1, p}\left(A_{\alpha}\right) .
$$

Note that $(\mathbf{z}, \mathbf{f})$ is a nodal $\mathbf{J}$-holomorphic map iff $\mathbf{f} \in\left(\bar{\partial}_{J}, \mathrm{ev}^{E}\right)^{-1}\left(0_{\mathcal{E}} \times\right.$ $\left.\Delta^{E}\right) \subset \mathcal{B}\left(\left\{A_{\alpha}\right\}\right)$, where $0_{\mathcal{E}}$ denotes the zero section of $\mathcal{E}\left(\left\{A_{\alpha}\right\}\right)$. We also have the $k$-point evaluation map

$$
\mathrm{ev}^{k}: \mathcal{B}\left(\left\{A_{\alpha}\right\}\right) \longrightarrow X^{k}, \quad \mathbf{f} \longmapsto\left(f_{\alpha_{1}}\left(z_{1}\right), \ldots, f_{\alpha_{k}}\left(z_{k}\right)\right) .
$$

Similarly, the reduced index set $R \subset\{1, \ldots, k\}$ gives rise to the reduced evaluation map

$$
\mathrm{ev}^{R}: \mathcal{B}\left(\left\{A_{\alpha}\right\}\right) \longrightarrow X^{R}, \quad \mathbf{f} \longmapsto\left\{f_{\alpha_{i}}\left(z_{i}\right)\right\}_{i \in R} .
$$

We first consider the case $\mathcal{B}(\{0\})$ in which $A_{\alpha}=0$ for all $\alpha \in T$. Then the whole tree $T$ is a ghost tree and therefore $R=\{1\}$.

Lemma 5.4. Let $T$ be a 1-labelled tree and $\mathbf{J} \in \mathcal{J}_{T}$. Then for any $x \in X$,

$$
\left(\bar{\partial}_{\mathbf{J}}, \mathrm{ev}^{E}, \mathrm{ev}^{1}\right): \mathcal{B}(\{0\}) \longrightarrow \mathcal{E}(\{0\}) \times X^{E} \times X
$$

is transverse to $0_{\mathcal{E}} \times \Delta^{E} \times\{x\}$.

Proof. Let $\mathbf{f} \in\left(\bar{\partial}_{\mathbf{J}}, \mathrm{ev}^{E}, \mathrm{ev}^{1}\right)^{-1}\left(0_{\mathcal{E}} \times \Delta^{E} \times\{x\}\right) \subset \mathcal{B}(\{0\})$. Since $\mathbf{f}$ has zero energy, this implies $f_{\alpha} \equiv x$ for all $\alpha \in T$. The linearized operator at $\mathbf{f}$ is given by

$$
\begin{gathered}
T_{\mathbf{f}} \mathcal{B} \longrightarrow \mathcal{E}_{\mathbf{f}} \oplus\left(T_{x} X\right)^{E} \oplus T_{x} X, \\
\left\{\xi_{\alpha}\right\}_{\alpha \in T} \longmapsto\left(\left\{\bar{\partial}_{\alpha} \xi_{\alpha}\right\}_{\alpha \in T},\left\{\xi_{\alpha}\left(z_{\alpha \beta}\right)\right\}_{\alpha E \beta}, \xi_{\alpha_{1}}\left(z_{1}\right)\right),
\end{gathered}
$$

where $\bar{\partial}_{\alpha}: T_{f_{\alpha}} \mathcal{B} \rightarrow \mathcal{E}_{f_{\alpha}}$ is the linearized Cauchy-Riemann operator. By Lemma $4.2, \bar{\partial}_{\alpha}$ is surjective and its kernel consists of the constant functions 
$\xi_{\alpha}: S_{\alpha} \rightarrow T_{x} X$. Thus it suffices to show that the restriction to the kernel of the $\bar{\partial}_{\alpha}$

$$
\left(T_{x} X\right)^{T} \longrightarrow\left(T_{x} X\right)^{E} \oplus T_{x} X, \quad\left\{\xi_{\alpha}\right\}_{\alpha \in T} \longmapsto\left(\left\{\xi_{\alpha}\right\}_{\alpha E \beta}, \xi_{\alpha_{1}}\right)
$$

is transverse to the subspace $T_{x E} \Delta^{E} \oplus\{0\} \subset\left(T_{x} X\right)^{E} \oplus T_{x} X$. This is equivalent to the surjectivity of the operator

$$
\begin{gathered}
\left(T_{x} X\right)^{T} \oplus T_{x^{E}} \Delta^{E} \longrightarrow\left(T_{x} X\right)^{E} \oplus T_{x} X, \\
\left(\left\{\xi_{\alpha}\right\}_{\alpha \in T},\left\{\eta_{\alpha \beta}\right\}_{\alpha E \beta}\right) \longmapsto\left(\left\{\xi_{\alpha}+\eta_{\alpha \beta}\right\}_{\alpha E \beta}, \xi_{\alpha_{1}}\right) .
\end{gathered}
$$

Thus for given $\left\{v_{\alpha \beta}\right\}_{\alpha E \beta}$ and $v_{1}$, we need to find $\left\{\xi_{\alpha}\right\}_{\alpha \in T}$ and $\left\{\eta_{\alpha \beta}\right\}_{\alpha E \beta}$ satisfying

$$
\xi_{\alpha}+\eta_{\alpha \beta}=v_{\alpha \beta}, \quad \xi_{\alpha_{1}}=v_{1}, \quad \eta_{\alpha \beta}=\eta_{\beta \alpha}
$$

for all $\alpha E \beta$. This system can be solved by induction as follows. Let $T_{\ell}$ be the set of vertices that have distance at most $\ell$ from $\alpha_{1}$. For $\ell=0$ set $\xi_{\alpha_{1}}:=v_{1}$. For the induction step, suppose that we have a solution of the system (5.3) for all $\alpha E \beta$ with $\alpha, \beta \in T_{\ell}$. Since $T$ is a tree, for $\beta \in T_{\ell+1} \backslash T_{\ell}$ there exists a unique $\alpha \in T_{\ell}$ with $\alpha E \beta$. Then $\eta_{\alpha \beta}:=\eta_{\beta \alpha}:=v_{\alpha \beta}-\xi_{\alpha}$ and $\xi_{\beta}:=v_{\beta \alpha}-\eta_{\beta \alpha}$ solves (5.3) on $T_{\ell+1}$.

More generally, let $k \geq 3$ and let $I$ be a subset of $\{1, \ldots, k\}$ with $|I| \geq 3$. Call a $k$-labelled tree $T I$-stable if it is still stable after removing the marked points not in $I$. Note that $I$-stability implies stability. Denote by

$$
\pi_{I}: \overline{\mathcal{M}}_{k+1} \longrightarrow \overline{\mathcal{M}}_{|I|+1}
$$

the obvious projection (forgetting the marked points outside the set $I \cup\{0\}$ and stabilizing).

Fix $J_{0} \in \mathcal{J}$ and a nonempty open subset $V \subset X$ and set

$$
\begin{gathered}
\mathcal{J}(V):=\left\{J \in \mathcal{J} \mid J=J_{0} \text { outside } V\right\}, \\
\mathcal{J}_{k+1}(V):=\left\{J \in \mathcal{J}_{k+1} \mid J=J_{0} \text { outside } V\right\} .
\end{gathered}
$$

By Lemma 3.6, a $J \in \mathcal{J}_{|I|+1}(V)$ induces a coherent map $\pi_{I}^{*} J: \overline{\mathcal{M}}_{k+1} \supset \pi^{-1}$ $\left(\mathcal{M}_{T}\right) \rightarrow \mathcal{J}(V)$ for each $I$-stable $k$-labelled tree $T$. We will often denote the pullback $\pi_{I}^{*} J$ again by $J$.

Fix a stable curve $\mathbf{z}$ modelled over the $k$-labelled tree $T$ and homology classes $A_{\alpha}, \alpha \in T$. We have the universal Cauchy-Riemann operator

$$
\bar{\partial}: \mathcal{B}\left(\left\{A_{\alpha}\right\}\right) \times \mathcal{J}_{|I|+1}(V) \longrightarrow \mathcal{E}\left(\left\{A_{\alpha}\right\}\right), \quad(\mathbf{f}, J) \longmapsto \bar{\partial}_{J_{\mathbf{z}}} \mathbf{f} .
$$

Definition 5.5. Denote by $\mathcal{B}^{*}\left(\left\{A_{\alpha}\right\}\right) \subset \mathcal{B}\left(\left\{A_{\alpha}\right\}\right)$ the open subset of maps f for which the image of each nonconstant $f_{\alpha}$ meets $V$. 
Lemma 5.6. For any I-stable curve $\mathbf{z}$ and $x \in X^{R}$

$$
\left(\bar{\partial}, \mathrm{ev}^{E}, \mathrm{ev}^{R}\right): \mathcal{B}^{*}\left(\left\{A_{\alpha}\right\}\right) \times \mathcal{J}_{|I|+1}(V) \longrightarrow \mathcal{E}\left(\left\{A_{\alpha}\right\}\right) \times X^{E} \times X^{R}
$$

is transverse to $0_{\mathcal{E}} \times \Delta^{E} \times\{x\}$.

Proof. Let $\mathbf{f} \in \mathcal{B}^{*}\left(\left\{A_{\alpha}\right\}\right)$ and $J \in \mathcal{J}_{|I|+1}(V)$ satisfy $\bar{\partial}_{J_{\mathbf{z}}} \mathbf{f}=0$. The linearization of $\left(\bar{\partial}, \mathrm{ev}^{E}, \mathrm{ev}^{R}\right)$ at $(f, J)$ is the linear operator

$$
\begin{gathered}
\bigoplus_{\alpha \in T} T_{f_{\alpha}} \mathcal{B} \oplus T_{J} \mathcal{J}_{|I|+1} \longrightarrow \bigoplus_{\alpha \in T} \mathcal{E}_{f_{\alpha}} \oplus \bigoplus_{\alpha E \beta} T_{f_{\alpha}\left(z_{\alpha \beta}\right)} X \oplus \bigoplus_{i \in R} T_{f_{\alpha_{i}}\left(z_{i}\right)} X, \\
\left(\left\{\xi_{\alpha}\right\}, Y\right) \longmapsto\left(\left\{D_{f_{\alpha}} \xi_{\alpha}+\frac{1}{2} Y_{\mathbf{z}} \circ d f_{\alpha} \circ j\right\},\left\{\xi_{\alpha}\left(z_{\alpha \beta}\right)\right\},\left\{\xi_{\alpha_{i}}\left(z_{i}\right)\right\}\right) .
\end{gathered}
$$

Note that this operator only depends on the restrictions of $Y$ to the $S_{\alpha}$. Let $T^{\mathrm{nc}}:=\left\{\alpha \in T \mid f_{\alpha} \neq\right.$ constant $\}$. Since $\mathbf{f} \in \mathcal{B}^{*}\left(\left\{A_{\alpha}\right\}\right)$, we find nonempty open sets $U_{\alpha} \subset f_{\alpha}^{-1}(V)$ for $\alpha \in T^{\text {nc }}$ that are disjoint in $\Sigma_{\mathbf{z}}$. Consider the space

$$
\bigoplus_{\alpha \in T} T_{J_{\alpha}} \mathcal{J}_{U_{\alpha}}
$$

where $J_{\alpha}=\left.J\right|_{S_{\alpha}}$ and for $\alpha \notin T^{\mathrm{nc}}$ we have set $U_{\alpha}:=\emptyset$ and $T_{J_{\alpha}} \mathcal{J}_{U_{\alpha}}:=\{0\}$. By Lemma 3.8, each element $\left\{Y_{\alpha}\right\} \in \bigoplus_{\alpha \in T} T_{J_{\alpha}} \mathcal{J}_{U_{\alpha}}$ extends to an element $Y \in T_{J} \mathcal{J}_{|I|+1}$. By the choice of the $U_{\alpha}$, the extension can be chosen so that $Y \in T_{J} \mathcal{J}_{|I|+1}(V)$. Composing with the corresponding injective linear map (see Lemma 3.8) it suffices to prove transversality to $T\left(0_{\mathcal{E}} \times \Delta^{E} \times\{x\}\right.$ ) of the linearized operator

$$
\begin{gathered}
L: \bigoplus_{\alpha \in T}\left[T_{f_{\alpha}} \mathcal{B} \oplus T_{J_{\alpha}} \mathcal{J}_{U_{\alpha}}\right] \longrightarrow \bigoplus_{\alpha \in T}\left[\mathcal{E}_{f_{\alpha}} \oplus \bigoplus_{\beta: \alpha E \beta} T_{f_{\alpha}\left(z_{\alpha \beta}\right)} X \oplus \bigoplus_{i: \alpha_{i}=\alpha} T_{f_{\alpha}\left(z_{i}\right)} X\right] \\
\left\{\left(\xi_{\alpha}, Y_{\alpha}\right)\right\} \longmapsto\left\{\left(D_{f_{\alpha}} \xi_{\alpha}+\frac{1}{2} Y_{\alpha} \circ d f_{\alpha} \circ j,\left\{\xi_{\alpha}\left(z_{\alpha \beta}\right)\right\},\left\{\xi_{\alpha}\left(z_{i}\right)\right\}\right)\right\} .
\end{gathered}
$$

Note that this operator is a direct sum of operators $L_{\alpha}$ (for $\alpha \in T$ ) of the form considered in Section 4. For $\alpha \in T^{\mathrm{nc}}, L_{\alpha}$ is surjective by Lemma 4.1. (Note that for $\alpha \notin T^{\mathrm{nc}}$ Lemma 4.1 is not applicable because $f_{\alpha}^{-1}(V)$ maybe empty.)

Next consider a maximal subtree $T^{\prime}=\left(T^{\prime}, E^{\prime}, \Lambda^{\prime}\right)$ contained in $T \backslash T^{\mathrm{nc}}$, i.e., $A_{\alpha}=0$ for all $\alpha \in T^{\prime}$. Thus $T^{\prime}$ is a ghost tree, so by definition of $R$ it contains at most one marked point in $R$. If a point $z_{i} \in R$ on $T^{\prime}$ exists denote by $\operatorname{ev}_{i}^{1}$ the evaluation map at this point and set $x_{i}:=\mathbf{f}\left(z_{i}\right)$. By Lemma 5.4, the map $\left(\bar{\partial}, \mathrm{ev}^{E^{\prime}}, \mathrm{ev}_{i}^{1}\right)$ is transverse to $0_{\mathcal{E}} \times \Delta^{E^{\prime}} \times\left\{x_{i}\right\}$ over $T^{\prime}$. Combining this with the surjectivity of $L_{\alpha}$ for $\alpha \in T^{\text {nc }}$, the transversality of $L$ to $T\left(0_{\mathcal{E}} \times\right.$ $\left.\Delta^{E} \times\{x\}\right)$ follows. 
For $J \in \mathcal{J}_{|I|+1}(V)$, denote by

$$
\tilde{\mathcal{M}}_{T}^{*}\left(\left\{A_{\alpha}\right\}, J\right) \subset \tilde{\mathcal{M}}_{T}\left(\left\{A_{\alpha}\right\}, J\right)
$$

the open subset of those $[\mathbf{z}, \mathbf{f}]$ for which $\mathbf{f} \in \mathcal{B}^{*}\left(\left\{A_{\alpha}\right\}\right)$.

Proposition 5.7. Let $T$ be an I-stable $k$-labelled tree, $A_{\alpha} \in H_{2}(X ; \mathbb{Z})$ for $\alpha \in T$, and $J_{0} \in \mathcal{J}$. Then the universal solution space

$$
\tilde{\mathcal{M}}_{T}^{*}\left(\left\{A_{\alpha}\right\}, \mathcal{J}_{|I|+1}(V)\right):=\bigcup_{J \in \mathcal{J}_{|I|+1}(V)} \tilde{\mathcal{M}}_{T}^{*}\left(\left\{A_{\alpha}\right\}, J\right)
$$

is a Banach manifold and the reduced evaluation map

$$
\mathrm{ev}^{R}: \tilde{\mathcal{M}}_{T}^{*}\left(\left\{A_{\alpha}\right\}, \mathcal{J}_{|I|+1}(V)\right) \longrightarrow X^{R}
$$

is a submersion.

Proof. By Lemma 5.6, for every $x \in X^{R}$ the linearization of the map

$$
\begin{gathered}
\tilde{\mathcal{M}}_{T} \times \mathcal{B}^{*}\left(\left\{A_{\alpha}\right\}\right) \times \mathcal{J}_{|I|+1}(V) \longrightarrow \mathcal{E}\left(\left\{A_{\alpha}\right\}\right) \times X^{E} \times X^{R}, \\
(\mathbf{z}, \mathbf{f}, J) \longmapsto\left(\bar{\partial}_{J_{\mathbf{z}}} \mathbf{f}, \mathrm{ev}^{E}(\mathbf{f}), \mathrm{ev}^{R}(\mathbf{f})\right) .
\end{gathered}
$$

is transverse to $T\left(0_{\mathcal{E}} \times \Delta^{E} \times\{x\}\right)$. Thus the preimage

$$
\left(\bar{\partial}, \mathrm{ev}^{E}\right)^{-1}\left(0_{\mathcal{E}} \times \Delta^{E}\right)=\tilde{\mathcal{M}}_{T}^{*}\left(\left\{A_{\alpha}\right\}, \mathcal{J}_{|I|+1}(V)\right)
$$

is a Banach manifold and the reduced evaluation map

$$
\mathrm{ev}^{R}: \tilde{\mathcal{M}}_{T}^{*}\left(\left\{A_{\alpha}\right\}, \mathcal{J}_{|I|+1}(V)\right) \longrightarrow X^{R}
$$

is a submersion.

In view of Lemma 5.1 and [19], the group $G_{T}$ of isomorphisms fixing the stable tree $T$ acts freely and properly on the space $\tilde{\mathcal{M}}_{T}^{*}\left(\left\{A_{\alpha}\right\}, \mathcal{J}_{|I|+1}(V)\right)$. Hence the universal moduli space

$$
\mathcal{M}_{T}^{*}\left(\left\{A_{\alpha}\right\}, \mathcal{J}_{|I|+1}(V)\right):=\tilde{\mathcal{M}}_{T}^{*}\left(\left\{A_{\alpha}\right\}, \mathcal{J}_{|I|+1}(V)\right) / G_{T}
$$

is a Banach manifold. Moreover, the reduced evaluation map descends to a submersion

$$
\mathrm{ev}^{R}: \mathcal{M}_{T}^{*}\left(\left\{A_{\alpha}\right\}, \mathcal{J}_{|I|+1}(V)\right) \longrightarrow X^{R} .
$$

For $J \in \mathcal{J}_{|I|+1}(V)$ define the moduli space

$$
\mathcal{M}_{T}^{*}\left(\left\{A_{\alpha}\right\}, J\right):=\tilde{\mathcal{M}}_{T}^{*}\left(\left\{A_{\alpha}\right\}, J\right) / G_{T}
$$

of $J$-holomorphic maps belonging to the set $\mathcal{B}^{*}\left(\left\{A_{\alpha}\right\}\right)$. For a smooth submanifold $Z \subset X^{R}$ define

$$
\mathcal{M}_{T}^{*}\left(\left\{A_{\alpha}\right\}, J ; Z\right):=\left\{[\mathbf{z}, \mathbf{f}] \in \mathcal{M}_{T}^{*}\left(\left\{A_{\alpha}\right\}, J\right) \mid\left\{f_{\alpha_{i}}\left(z_{i}\right)\right\}_{i \in R} \in Z\right\} .
$$


Corollary 5.8. Let $T$ be an I-stable k-labelled tree, $A_{\alpha} \in H_{2}(X ; \mathbb{Z})$ for $\alpha \in T, J_{0} \in \mathcal{J}$, and $Z \subset X^{R}$ a smooth submanifold. Then there exists a Baire set $\mathcal{J}_{|I|+1}^{\text {reg }}(V) \subset \mathcal{J}_{|I|+1}(V)$ (depending on $Z, T$ and the $A_{\alpha}$ ) such that for all $J \in \mathcal{J}_{|I|+1}^{\text {reg }}(V)$ the moduli space $\mathcal{M}_{T}^{*}\left(\left\{A_{\alpha}\right\}, J ; Z\right)$ is a smooth submanifold of $\mathcal{M}_{T}^{*}\left(\left\{A_{\alpha}\right\}, J\right)$ of dimension

$$
\operatorname{dim} \mathcal{M}_{T}^{*}\left(\left\{A_{\alpha}\right\}, J ; Z\right)=2 n-6+\sum_{\alpha \in T} 2 c_{1}\left(A_{\alpha}\right)+2 k-2 e(T)-\operatorname{codim}_{\mathbb{R}} Z .
$$

Proof. By the preceding discussion, the universal moduli space

$$
\mathcal{M}_{T}^{*}\left(\left\{A_{\alpha}\right\}, \mathcal{J}_{|I|+1}(V) ; Z\right):=\left(\mathrm{ev}^{R}\right)^{-1}(Z) \subset \mathcal{M}_{T}^{*}\left(\left\{A_{\alpha}\right\}, \mathcal{J}_{|I|+1}(V)\right)
$$

is a smooth Banach submanifold of $\mathcal{M}_{T}^{*}\left(\left\{A_{\alpha}\right\}, \mathcal{J}_{|I|+1}(V)\right)$ of codimension $\operatorname{codim}_{\mathbb{R}} Z$ with a smooth projection

$$
\pi: \mathcal{M}_{T}^{*}\left(\left\{A_{\alpha}\right\}, \mathcal{J}_{|I|+1}(V) ; Z\right) \longrightarrow \mathcal{J}_{|I|+1}(V), \quad(f, J) \longmapsto J .
$$

By the Sard-Smale theorem, there exists a Baire set $\mathcal{J}_{|I|+1}^{\text {reg }}(V) \subset \mathcal{J}_{|I|+1}(V)$ of regular values of the projection $\pi$. It follows that for $J \in \mathcal{J}_{|I|+1}^{\text {reg }}(V)$ the moduli space $\mathcal{M}_{T}^{*}\left(\left\{A_{\alpha}\right\}, J\right)$ is a smooth manifold and $\mathcal{M}_{T}^{*}\left(\left\{A_{\alpha}\right\}, J ; Z\right)$ is a smooth submanifold of codimension $\operatorname{codim}_{\mathbb{R}} Z$ in $\mathcal{M}_{T}^{*}\left(\left\{A_{\alpha}\right\}, J\right)$. Since, according to Lemma 5.6, $\mathcal{M}_{T}^{*}\left(\left\{A_{\alpha}\right\}, J\right)$ is transversely cut out by the edge evaluation map, its dimension follows from the usual dimension formula for its components and $|T|=e(T)+1$ :

$$
\begin{aligned}
\operatorname{dim} \mathcal{M}_{T}^{*}\left(\left\{A_{\alpha}\right\}, J\right) & =\sum_{\alpha \in T}\left(2 n-6+2 c_{1}\left(A_{\alpha}\right)\right)+2 k+4 e(T)-2 n e(T) \\
& =2 n-6|T|+2 \sum_{\alpha \in T} c_{1}\left(A_{\alpha}\right)+2 k+4 e(T) \\
& =2 n-6+\sum_{\alpha \in T} 2 c_{1}\left(A_{\alpha}\right)+2 k-2 e(T) .
\end{aligned}
$$

This implies the dimension formula in Corollary 5.8.

We conclude with an improvement of the dimension formula in Corollary 5.8 that will play a crucial role in Section 9 . If $|R|<k$ we have marked points on ghost components not belonging to $R$. Variations of these points contribute to the dimension formula but have no effect on the $J$-holomorphic maps (due to the coherency of $J$ ). So the "actual" dimension of the moduli space should be lower than the formula in Corollary 5.8. This statement can be made precise as follows. Evaluation at the points $\{1, \ldots, k\}$ induces a smooth evaluation map

$$
\mathrm{ev}^{k}: \mathcal{M}_{T}^{*}\left(\left\{A_{\alpha}\right\}, J ; Z\right) \longrightarrow X^{k}
$$

(which actually lands in a subset of $X^{k}$ because of the constraint $Z$ ). Let $\pi_{R}(T)$ be the stable $|R|$-labelled tree obtained from $T$ by forgetting the 
marked points not belonging to $R$ and stabilizing. On a nonghost component $\alpha$ this does not affect $J$ due to the coherency condition, so $J$-holomorphicity of the corresponding map $f_{\alpha}$ still makes sense. On a ghost component, the almost complex structure is no longer well defined (because $J$ depends on the positions of the forgotten points), but the condition $f_{\alpha}=$ const still makes sense. Hence forgetting the marked points outside $R$ yields a transversely cut out moduli space $\mathcal{M}_{\pi_{R}(T)}^{*}\left(\left\{A_{\alpha}\right\}, J ; Z\right)$. Transversality follows from Lemma 4.2. The notion of the moduli space differs slightly from the others and its elements are described by the preceding discussion. Since the image of a ghost tree $T^{\prime}$ is determined by the image of the marked point of $R$ on $T^{\prime}$, the evaluation map ev ${ }^{k}$ descends to this space and we have shown

Corollary 5.9. In the situation of Corollary 5.8, the evaluation map $\mathrm{ev}^{k}$ factors as

$$
\mathrm{ev}^{k}: \mathcal{M}_{T}^{*}\left(\left\{A_{\alpha}\right\}, J ; Z\right) \longrightarrow \mathcal{M}_{\pi_{R}(T)}^{*}\left(\left\{A_{\alpha}\right\}, J ; Z\right) \longrightarrow X^{k}
$$

through a smooth manifold of dimension

$$
\begin{aligned}
\operatorname{dim} \mathcal{M}_{\pi_{R}(T)}^{*}\left(\left\{A_{\alpha}\right\}, J ; Z\right)= & 2 n-6+\sum_{\alpha \in T} 2 c_{1}\left(A_{\alpha}\right)+2|R| \\
& -2 e\left(\pi_{R}(T)\right)-\operatorname{codim}_{\mathbb{R}} Z .
\end{aligned}
$$

Remark 5.10. The proofs show that Proposition 5.7, Corollaries 5.8 and 5.9 remain true if $\mathcal{J}_{|I|+1}(V)$ is replaced by the subset $\mathcal{J}_{|I|+1}^{*}(V) \subset \mathcal{J}_{|I|+1}(V)$ of those coherent $J$ mapping into some given open subset $\mathcal{J}^{*} \subset \mathcal{J}$.

\section{Tangencies}

In this section, we define moduli spaces of $J$-holomorphic spheres with prescribed orders of tangency to complex submanifolds and prove their regularity.

We begin with the local situation. Suppose $A: \mathbb{R}^{m} \rightarrow \mathbb{R}^{m \times m}$ is a smooth matrix valued function. Assume further that $A$ preserves a subspace $\mathbb{R}^{k}:=\mathbb{R}^{k} \times\{0\} \subset \mathbb{R}^{m}(0 \leq k \leq m-1)$ in the sense that $A(x)\left(\mathbb{R}^{k} \times\right.$ $\{0\}) \subset \mathbb{R}^{k} \times\{0\}$ for all $x \in \mathbb{R}^{k}$. Let $D \subset \mathbb{C}$ be the unit disk and $f: D \rightarrow \mathbb{R}^{m}$ be a smooth map. Denote coordinates on $\mathbb{C}$ by $z=s+i t$ and partial derivatives by $f_{s}, f_{\text {st }}$ etc. Denote by $d^{\ell} f$ the vector of all partial derivatives of order $i$ with $1 \leq i \leq \ell$. We say $d^{\ell} f \in \mathbb{R}^{k}$ if all the partial derivatives in $d^{\ell} f$ lie in the subspace $\mathbb{R}^{k}$. The following lemma is proved by a simple induction over $i+j$.

Lemma 6.1. For all integers $i, j \geq 0$ we have an equation

$$
\frac{\partial^{i+j}}{\partial s^{i} \partial t^{j}}\left(A(f) f_{s}\right)=A(f) \frac{\partial^{i+j+1} f}{\partial s^{i+1} \partial t^{j}}+A_{i, j}(f)\left(d^{i+j} f\right)
$$


where $A_{i, j}(f)$ is a multilinear function in the partial derivatives up to order $i+j$. Moreover, $A_{i, j}(f)\left(d^{i+j} f\right) \in \mathbb{R}^{k}$ if $f \in \mathbb{R}^{k}$ and $d^{i+j} f \in \mathbb{R}^{k}$.

We first apply Lemma 6.1 in the following situation. Let $J$ be an almost complex structure on $\mathbb{C}^{n}$. We say that $J$ preserves $\mathbb{C}^{k}:=\mathbb{C}^{k} \times\{0\} \subset \mathbb{C}^{n}$ if $J(x): \mathbb{C}^{k} \rightarrow \mathbb{C}^{k}$ for all $x \in \mathbb{C}^{k}$. Let $f: \mathbb{C} \supset D \rightarrow \mathbb{C}^{n}$ be a $J$-holomorphic map, i.e., $f_{s}+J(f) f_{t}=0$. Then $f_{t}=J(f) f_{s}$ and Lemma 6.1 (with $A=J$ ) yields for $i, j \geq 0$ :

$$
\frac{\partial^{i+j+1} f}{\partial s^{i} \partial t^{j+1}}=J(f) \frac{\partial^{i+j+1} f}{\partial s^{i+1} \partial t^{j}}+A_{i, j}(f)\left(d^{i+j} f\right) .
$$

Multiplying by $J$ we find

$$
\left(\partial_{s}+J(f) \partial_{t}\right) \frac{\partial^{i+j} f}{\partial s^{i} \partial t^{j}}=J_{i, j}(f)\left(d^{i+j} f\right),
$$

where $J_{i, j}(f):=J(f) A_{i, j}(f)$ satisfies $J_{i, j}(f)\left(d^{i+j} f\right) \in \mathbb{C}^{k}$ if $f \in \mathbb{C}^{k}$ and $d^{i+j}$ $f \in \mathbb{C}^{k}$. Thus partial derivatives of $f$ are $J$-holomorphic up to lower order terms. This equation allows us to swap one $s$-derivative for a $t$-derivative, and iterating this process we obtain

Corollary 6.2. Let $f:(D, 0) \rightarrow\left(\mathbb{C}^{n}, 0\right)$ be $J$-holomorphic with $J$ preserving $\mathbb{C}^{k}$. If $d^{\ell-1} f(0) \in \mathbb{C}^{k}$ and $\frac{\partial^{\ell} f}{\partial s^{\ell}}(0) \in \mathbb{C}^{k}$ for some $\ell \geq 1$, then $d^{\ell} f(0) \in \mathbb{C}^{k}$.

If $f(0)=0$ and $d^{\ell-1} f(0) \in \mathbb{C}^{k}$ we define the equivalence class

$$
j_{\mathbb{C}^{k}}^{\ell} f(0):=\left[\frac{\partial^{\ell} f}{\partial s^{\ell}}(0)\right] \in \mathbb{C}^{n} / \mathbb{C}^{k}
$$

By Corollary $6.2, j_{\mathbb{C}^{k}}^{\ell} f(0)=0$ implies $d^{\ell} f(0) \in \mathbb{C}^{k}$. So $j_{\mathbb{C}^{k}}^{\ell+1} f(0)$ is defined whenever $f(0)=0$ and $j_{\mathbb{C}^{k}}^{1} f(0)=\cdots=j_{\mathbb{C}^{k}}^{\ell} f(0)=0$.

Next we study how $j_{\mathbb{C}^{k}}^{\ell} f(0)$ transforms under diffeomorphisms $\phi:\left(\mathbb{C}^{n}, 0\right) \rightarrow$ $\left(\mathbb{C}^{n}, 0\right)$ with $\phi\left(\mathbb{C}^{k}\right) \subset \mathbb{C}^{k}$. We have $(\phi \circ f)_{s}=D \phi(f) f_{s}$, so Lemma 6.1 (with $A=D \phi)$ yields

$$
\frac{\partial^{i+j+1}(\phi \circ f)}{\partial s^{i+1} \partial t^{j}}=D \phi(f) \frac{\partial^{i+j+1} f}{\partial s^{i+1} \partial t^{j}}+A_{i, j}(f)\left(d^{i+j} f\right) .
$$

Of course, the same argument applies to the $t$-derivative and so we find for all $i+j \geq 1$ :

$$
\frac{\partial^{i+j}(\phi \circ f)}{\partial s^{i} \partial t^{j}}=D \phi(f) \frac{\partial^{i+j} f}{\partial s^{i} \partial t^{j}}+\phi_{i, j}(f)\left(d^{i+j-1} f\right),
$$

where $\phi_{i, j}(f)$ satisfies $\phi_{i, j}(f)\left(d^{i+j-1} f\right) \in \mathbb{C}^{k}$ if $f \in \mathbb{C}^{k}$ and $d^{i+j-1} f \in \mathbb{C}^{k}$. This proves 
Corollary 6.3. Let $f:(D, 0) \rightarrow\left(\mathbb{C}^{n}, 0\right)$ be a smooth map and $\phi:\left(\mathbb{C}^{n}, 0\right) \rightarrow$ $\left(\mathbb{C}^{n}, 0\right)$ a diffeomorphism preserving $\mathbb{C}^{k}$. If $d^{\ell-1} f(0) \in \mathbb{C}^{k}$ for some $\ell \geq 1$, then

$$
\left[\frac{\partial^{\ell}(\phi \circ f)}{\partial s^{\ell}}(0)\right]=\left[D \phi(0) \frac{\partial^{\ell} f}{\partial s^{\ell}}(0)\right] \in \mathbb{C}^{n} / \mathbb{C}^{k}
$$

Corollary 6.3 shows that if $f:(D, 0) \rightarrow\left(\mathbb{C}^{n}, 0\right)$ is $J$-holomorphic and $j_{\mathbb{C}^{k}}^{1} f(0)=\cdots=j_{\mathbb{C}^{k}}^{\ell-1} f(0)=0$, then $j_{\mathbb{C}^{k}}^{\ell} f(0)$ transforms like a normal vector to the submanifold $\mathbb{C}^{k} \subset \mathbb{C}^{n}$.

Now it is clear how to globalize these constructions. Let $Z$ be a $k$ dimensional (almost) complex submanifold of an almost complex manifold $(X, J)$. Let $f: \mathbb{C} \supset D \rightarrow X$ be a $J$-holomorphic map with $f(0)=x \in Z$. Choose a coordinate chart $\psi$ for $X$ mapping $x$ to 0 and $Z$ to $\mathbb{C}^{k}$. We say that $f$ is tangent to order $\ell \geq 0$ to $Z$ at 0 , and write $d^{\ell} f(0) \in T_{x} Z$, if $d^{\ell}(\psi \circ f)(0) \in \mathbb{C}^{k}$. By the discussion preceding Corollary 6.3 this definition is independent of the coordinate chart $\psi$.

Suppose that for some $\ell \geq 1$ we have $d^{\ell-1} f(0) \in T_{x} Z$. Then we define the $\ell$-jet normal to $Z$ of $f$ at 0 as

$$
j_{Z}^{\ell} f(0):=D \psi(x)^{-1} j^{\ell}(\psi \circ f)(0) \in T_{x} X / T_{x} Z .
$$

By Corollary 6.2, $j_{Z}^{\ell} f(0)$ is defined whenever $j_{Z}^{1} f(0)=\cdots=j_{Z}^{\ell-1} f(0)=0$ and by Corollary 6.3, the definition of $j_{Z}^{\ell} f(0)$ does not depend on the coordinate chart $\psi$. So we have proved

Lemma 6.4. Let $Z$ be a complex submanifold of an almost complex manifold $(X, J)$ and $f: \mathbb{C} \supset D \rightarrow X$ be a J-holomorphic map with $f(0)=x \in Z$. Then there is a sequence $j_{Z}^{1} f(0), j_{Z}^{2} f(0), \ldots, \in T_{x} X / T_{x} Z$ of normal derivatives such that the $\ell$ th normal derivative $j_{Z}^{\ell} f(0)$ is defined whenever $j_{Z}^{1} f(0)=\cdots=j_{Z}^{\ell-1} f(0)=0$. Moreover, $f$ is tangent to order $\ell$ to $Z$ at 0 if and only if $j_{Z}^{1} f(0)=\cdots=j_{Z}^{\ell} f(0)=0$.

Now let $(X, \omega)$ be a symplectic manifold with a tamed almost complex structure $J_{0}$ and let $Z_{1}, \ldots, Z_{k}$ be $k$ complex submanifolds of $\left(X, J_{0}\right)$. Fix a nonempty open subset $V \subset X$ with $V \cap\left(Z_{1} \cup \cdots \cup Z_{k}\right)=\emptyset$ and denote by

$$
\mathcal{J}(V):=\left\{J \in \mathcal{J}(X, \omega) \mid J=J_{0} \text { on } X \backslash V\right\}
$$

the space of tamed almost complex structures that agree with $J_{0}$ outside $V$. Note that $Z_{1}, \ldots, Z_{k}$ are $J$-complex submanifolds for all $J \in \mathcal{J}(V)$.

Fix non-negative integers $\ell_{1}, \ldots, \ell_{k}$ and pairwise disjoint points $z_{1}, \ldots, z_{k}$ $\in S^{2}$. Denote by $\mathbf{z}:=\left(z_{1}, \ldots, z_{k}\right)$ their collection Also choose holomorphic coordinate charts for $S^{2}$ around the $z_{i}$ mapping $z_{i}$ to $0 \in \mathbb{C}$. For $m-2 / p>$ 
$\max \left\{\ell_{i}\right\}$ define the universal solution space

$$
\begin{aligned}
\tilde{\mathcal{M}}_{\mathbf{z}}:=\tilde{\mathcal{M}}_{\mathbf{z}}\left(\mathcal{J}(V) ;\left\{Z_{i}, \ell_{i}\right\}\right):=\{ & (f, J) \in \mathcal{B}^{m, p} \times \mathcal{J}(V) \mid \bar{\partial}_{J} f=0, \\
& \left.d^{\ell_{i}} f\left(z_{i}\right) \in T_{f\left(z_{i}\right)} Z_{i} \text { for } i=1, \ldots, k\right\}
\end{aligned}
$$

of holomorphic maps tangent to order $\ell_{i}$ to $Z_{i}$ at $z_{i}$. Notice that this definition does not depend on the choice of holomorphic coordinates around the $z_{i}$. Denote by

$$
\tilde{\mathcal{M}}_{\mathbf{z}}^{*}\left(\mathcal{J}(V) ;\left\{Z_{i}, \ell_{i}\right\}\right):=\left\{(f, J) \in \tilde{\mathcal{M}}_{\mathbf{z}}\left(\mathcal{J}(V) ;\left\{Z_{i}, \ell_{i}\right\}\right) \mid f^{-1}(V) \neq \emptyset\right\}
$$

the subspace of solutions passing through $V$, and by

$$
\tilde{\mathcal{M}}_{\mathbf{z}}^{s}:=\tilde{\mathcal{M}}_{\mathbf{z}}^{s}\left(\mathcal{J}(V) ;\left\{Z_{i}, \ell_{i}\right\}\right):=\left\{(f, J) \in \tilde{\mathcal{M}}_{\mathbf{z}}^{*}\left(\mathcal{J}(V) ;\left\{Z_{i}, \ell_{i}\right\}\right) \mid f \text { simple }\right\}
$$

the subspace of simple solutions passing through $V$. Here a holomorphic map is called simple if it is not a nontrivial branched covering of another holomorphic map. We wish to show that $\tilde{\mathcal{M}}_{\mathrm{z}}^{s}$ is a Banach manifold. So consider $(f, J) \in \tilde{\mathcal{M}}_{\mathbf{z}}^{s}$. Choose the coordinate charts $\phi_{i}$ for $X$ around $f\left(z_{i}\right)$ mapping $f\left(z_{i}\right)$ to 0 and $Z_{i}$ to $\mathbb{C}^{n_{i}} \subset \mathbb{C}^{n}$. Using $\phi_{i}$, we view $f(z)$ for $z$ near $z_{i}$ as a point in $\mathbb{C}^{n}$. Then the candidate for the tangent space of $\tilde{\mathcal{M}}_{\mathbf{z}}^{s}$ at $(f, J)$ is

$$
\begin{aligned}
& T_{(f, J)} \tilde{\mathcal{M}}_{\mathbf{z}}^{s}:=\left\{(\xi, Y) \in T_{f} \mathcal{B}^{m, p} \times T_{J} \mathcal{J}(V) \mid D_{f} \xi+\frac{1}{2} Y(f) \circ d f \circ j=0,\right. \\
&\left.d^{\ell_{i}} \xi\left(z_{i}\right) \in \mathbb{C}^{n_{i}} \text { for } i=1, \ldots, k\right\},
\end{aligned}
$$

where $\xi$ is viewed as a $\mathbb{C}^{n}$-valued function near $z_{i}$ via $d \phi_{i}$. If in addition $m-2 / p>\ell_{j}+1$ for some $j$, then in view of Lemma 6.4 (with respect to the fixed coordinate chart for $S^{2}$ near $\left.z_{j}\right)$ we have a normal $\left(\ell_{j}+1\right)$-jet evaluation map to the normal bundle $T X / T Z_{j}$ of $Z_{j}$ in $X$,

$$
j_{Z_{j}}^{\ell_{j}+1} \mathrm{ev}_{j}: \tilde{\mathcal{M}}_{\mathbf{z}}^{s} \longrightarrow T X / T Z, \quad(f, J) \longmapsto j_{Z_{j}}^{\ell_{j}+1} f\left(z_{j}\right) .
$$

With respect to the trivializations above, the linearization of $j_{Z_{j}}^{\ell_{j}+1} \mathrm{ev}_{j}$ at $(f, J)$ is the linear operator

$$
L_{j}: T_{(f, J)} \tilde{\mathcal{M}}_{\mathbf{z}}^{s} \longrightarrow \mathbb{C}^{n} / \mathbb{C}^{k_{j}}, \quad(\xi, Y) \longmapsto j_{\mathbb{C}^{n_{j}}}^{\ell_{j}+1} \xi\left(z_{j}\right) .
$$

\section{Lemma 6.5.}

(a) For $m-2 / p>\max \left\{\ell_{i}\right\}$ the space $\tilde{\mathcal{M}}_{\mathbf{z}}^{s}\left(\mathcal{J}(V) ;\left\{Z_{i}, \ell_{i}\right\}\right)$ is a smooth Banach manifold whose tangent space at $(f, J)$ is given by (6.1).

(b) Suppose that, in addition, $m-2 / p>\ell_{j}+1$ for some $j$ with $\ell_{j} \geq \max \left\{\ell_{i}\right\}-1$. Then the linearized normal $\left(\ell_{i}+1\right)$-jet evaluation map $L_{j}$ given by $(6.2)$ is surjective. 
We will give the proof Lemma 6.5 below. As the proof of Lemma 4.1, it is an adaptation of the proof of [19, Proposition 3.4.2]. Note that besides the presence of higher-order jets, Lemma 6.5 also differs from Lemma 4.1 in that it uses only domain-independent almost complex structures (which forces us to restrict to simple holomorphic maps).

The proof is based on the following lemma. Here we view $\eta \in \mathcal{E}_{f}^{m-1, p}$ near $z_{i}$ via the trivialization near $f\left(z_{i}\right)$ as a map $\mathbb{C} \supset U_{i} \rightarrow \mathbb{C}^{n}$, the corresponding $(0,1)$-form being given by $\eta d s-J(f) \eta d t$ (cf. [19, Section 3.1]). This allows us to speak of the $\ell$-jets $d^{\ell} \eta\left(z_{i}\right)$ at the $z_{i}$. Note that the spaces in Lemma 6.6 are well defined by the Sobolev embedding theorem. We point out that in Lemma 6.6, we consider the full $\ell$-jets (not just those normal to the $Z_{i}$ ) and the integer $\ell$ is entirely unrelated to the $\ell_{i}$.

Lemma 6.6. Let $(f, J) \in \tilde{\mathcal{M}}_{\mathbf{z}}^{s}\left(\mathcal{J}(V) ;\left\{Z_{i}, \ell_{i}\right\}\right)$. For $\ell \geq 0$ and $m-2 / p>\ell+1$ define the spaces

$$
\begin{aligned}
& B_{0}^{m, p}:=\left\{\xi \in T_{f} \mathcal{B}^{m, p} \mid d^{\ell+1} \xi\left(z_{i}\right)=0 \text { for } i=1, \ldots, k\right\} \\
& E_{0}^{m-1, p}:=\left\{\eta \in \mathcal{E}_{f}^{m-1, p} \mid d^{\ell} \eta\left(z_{i}\right)=0 \text { for } i=1, \ldots, k\right\} .
\end{aligned}
$$

Then the linear operator

$$
F_{0}: B_{0}^{m, p} \oplus T_{J} \mathcal{J}(V) \rightarrow E_{0}^{m-1, p}, \quad(\xi, Y) \longmapsto D_{f} \xi+\frac{1}{2} Y(f) \circ d f \circ j
$$

is surjective.

Proof. The corresponding statement for $\ell=-1$ was shown in the proof of [19, Lemma 3.4.3], see also the proof of Lemma 4.1 above. However, unlike in the case $\ell=-1$, for $\ell \geq 0$ we cannot reduce the result to the case $m=1$ because the $(\ell+1)$-jet $d^{\ell+1} \xi\left(z_{i}\right)$ at a point is not well defined in the Sobolev space $W^{1, p}$. This forces us to work with distributions instead of functions.

Recall that $T_{f} \mathcal{B}^{m, p}=W^{m, p}\left(S^{2}, B\right)$ and $\mathcal{E}_{f}^{m-1, p}=W^{m-1, p}\left(S^{2}, E\right)$ are spaces of Sobolev sections in the vector bundles $B:=f^{*} T X \rightarrow S^{2}$ and $E:=\Lambda^{0,1}$ $\left(f^{*} T X\right) \rightarrow S^{2}$.

Suppose first that $\ell+1<m-2 / p<\ell+2$. Let us view $F_{0}$ as an operator from $B_{0}^{m, p}$ to $\mathcal{E}_{f}^{m-1, p}$. Since $D_{f}$ is a Fredholm operator, the image im $F_{0}$ of $F_{0}$ is closed in $\mathcal{E}_{f}^{m-1, p}$. We will prove the inclusion $\left(\mathrm{im} F_{0}\right)^{\perp} \subset\left(E_{0}^{m-1, p}\right)^{\perp}$ in the dual space $\left(\mathcal{E}_{f}^{m-1, p}\right)^{*}$. In view of reflexivity of the Sobolev space $\mathcal{E}_{f}^{m-1, p}$ (see e.g., [1]), closedness of im $F_{0}$, and the Hahn-Banach theorem, this implies the desired statement $E_{0}^{m-1, p} \subset \operatorname{im} F_{0}$. Note that for $m>1,\left(\mathcal{E}_{f}^{m-1, p}\right)^{*}$ is the space of distributions $\Lambda \in C^{\infty}\left(S^{2}, E\right)^{*}$ that satisfy an inequality

$$
|\Lambda(\phi)| \leq C\|\phi\|_{W^{m-1, p}}
$$


for all smooth sections $\phi: S^{2} \rightarrow E$. Suppose that $\Lambda$ vanishes on $\operatorname{im}\left(F_{0}\right)$, i.e.,

$$
\Lambda\left(D_{f} \xi+\frac{1}{2} Y(f) \circ d f \circ j\right)=0
$$

for all $(\xi, Y) \in B_{0}^{m, p}$. In particular, this implies that $\Lambda\left(D_{f} \xi\right)=0$ for all smooth sections $\xi: S^{2} \rightarrow B$ with compact support in $S^{*}:=S^{2} \backslash\left\{z_{1}, \ldots, z_{k}\right\}$. Elliptic regularity for distributions (see [21, Theorem 8.12] for the case with constant coefficients and [6, Chapter XIV, Theorem 6.2., p. 1704] for the general case) implies that the restriction of $\Lambda$ to $S^{*}$ is represented by a smooth section $\eta: S^{*} \rightarrow E$ such that

$$
\Lambda(\phi)=\langle\phi, \eta\rangle_{L^{2}}
$$

for all $\phi \in C_{0}^{\infty}\left(S^{*}, B\right)$. Moreover, $\eta$ satisfies the Cauchy-Riemann type equation $D_{f}^{*} \eta=0$ and the equation

$$
\langle Y(f) \circ d f \circ j, \eta\rangle=0
$$

for all $Y \in T_{J} \mathcal{J}(V)$. (Note that $Y(f)$ has compact support in $S^{*}$ because $V$ is disjoint from the $Z_{i}$ ).

By definition of $\tilde{\mathcal{M}}_{\mathbf{z}}^{s}(f$ is simple!) and [19, Proposition 2.5.1], the set $U:=f^{-1}(V)$ is nonempty and the set $U^{*}:=\left\{z \in U \mid d f(z) \neq 0, f^{-1}\right.$ $(f(z))=\{z\}\}$ of injective points is open and dense in $U$. Suppose that $\eta(z) \neq 0$ for some $z \in U^{*}$. Then as in the proof of [19, Lemma 3.4.3], we find a $Y \in T_{J} \mathcal{J}(V)$ such that $\left\langle Y(f) \circ d f\left(z^{\prime}\right) \circ j, \eta\left(z^{\prime}\right)\right\rangle \geq 0$ for all $z^{\prime}$ and $>0$ at $z^{\prime}=z$, in contradiction to Equation (6.3). It follows that $\eta(z)=0$ for all $z \in U^{*}$, and hence by unique continuation (see [19, Lemma 3.4.7]) $\eta \equiv 0$ on $S^{*}$.

This proves that the distribution $\Lambda$ has support in the finite set $\left\{z_{1}, \ldots, z_{k}\right\}$. By $\left[\mathbf{2 1}\right.$, Theorem 6.25], there exist $\mathbb{R}$-linear functionals $c_{\alpha}^{i}: E_{z_{i}} \cong$ $\mathbb{C}^{n} \rightarrow \mathbb{R}$ such that

$$
\Lambda=\sum_{i=1}^{k} \sum_{|\alpha| \leq N} c_{\alpha}^{i} D^{\alpha} \delta_{z_{i}}
$$

Here $\alpha=\left(\alpha_{1}, \alpha_{2}\right) \in \mathbb{N}_{0}^{2}$ are multi-indices, $D^{\alpha}=\left(\partial^{\alpha_{1}+\alpha_{2}}\right) /\left(\partial s^{\alpha_{1}} \partial t^{\alpha_{2}}\right)$ are partial derivatives, $\delta_{z_{i}}$ is the delta distribution at the point $z_{i}$, and $D^{\alpha} \delta_{z_{i}}(\phi)=$ $(-1)^{|\alpha|} D^{\alpha} \phi\left(z_{i}\right)$ for $\phi \in C^{\infty}\left(S^{2}, E\right)$. The integer $N$ in (6.4) is the order of $\Lambda$, which is by definition the smallest integer such that $|\Lambda(\phi)| \leq C\|\phi\|_{C^{k}}$ for all $\phi \in C^{\infty}\left(S^{2}, E\right)$. For $\Lambda \in\left(\mathcal{E}_{f}^{m-1, p}\right)^{*}$ we clearly have $N \leq m-1$. More precisely, $\Lambda \in\left(\mathcal{E}_{f}^{m-1, p}\right)^{*}$ implies that for every $c_{\alpha}^{i} \neq 0$ in (6.4) we must have

$$
\left|D^{\alpha} \phi\left(z_{i}\right)\right| \leq C\|\phi\|_{W^{m-1, p}}
$$


for all $\phi \in C^{\infty}\left(S^{2}, E\right)$. It follows from sharpness of the Sobolev embedding theorem that $|\alpha| \leq[m-1-2 / p]$ (if $|\alpha|>[m-1-2 / p]$ there exist smooth functions $\phi$ with $W^{m-1, p}$-norm 1 and arbitrarily large $\left.\left|D^{\alpha} \phi\left(z_{i}\right)\right|\right)$. The assumption $\ell+1<m-2 / p<\ell+2$ made above implies $[m-1-2 / p]=\ell$, so we have shown

$$
\Lambda=\sum_{i=1}^{k} \sum_{|\alpha| \leq \ell} c_{\alpha}^{i} D^{\alpha} \delta_{z_{i}}
$$

But this means that

$$
\Lambda(\phi)=\sum_{i=1}^{k} \sum_{|\alpha| \leq \ell} c_{\alpha}^{i}(-1)^{|\alpha|} D^{\alpha} \phi\left(z_{i}\right)=0
$$

for all $\phi \in E_{0}^{m-1, p}$, so $\Lambda \in\left(E_{0}^{m-1, p}\right)^{\perp}$. This proves $\left(\operatorname{im} F_{0}\right)^{\perp} \subset\left(E_{0}^{m-1, p}\right)^{\perp}$ and hence, in view of the discussion above, the lemma in the special case $\ell+1<$ $m-2 / p<\ell+2$.

The case of general $m, p$ with $m-2 / p>\ell+1$ follows from the special case by elliptic regularity. Choose $m^{\prime} \leq m$ and $p^{\prime} \leq p$ such that $\ell+1<$ $m^{\prime}-2 / p^{\prime}<\ell+2$. Given $\eta \in E_{0}^{m-1, p} \subset E_{0}^{m^{\prime}-1, p^{\prime}}$, by the special case we find $(\xi, Y) \in B_{0}^{m^{\prime}, p^{\prime}} \oplus T_{J} \mathcal{J}(V)$ with $F_{0}(\xi, Y)=\eta$, and elliptic regularity ([19, Theorem C.2.3]) yields $\xi \in B_{0}^{m, p}$. This concludes the proof of Lemma 6.6.

Proof of Lemma 6.5. Part (a) in Lemma 6.5 in the case $\ell_{1}=\cdots=\ell_{k}=0$ follows from [19, Proposition 3.4.2]. Moreover, in view of Lemma 6.4, parts (a) and (b) of Lemma 6.5 for $\ell_{1}, \ldots, \ell_{k}$ imply part (a) with $\ell_{j}$ replaced by $\ell_{j}+1$. Hence by induction (always preserving the condition $\ell_{j} \geq \max \left\{\ell_{i}\right\}-1$ ), it suffices to prove part (b).

As above (cf. [19, Section 3.1]), we identify $(0,1)$-forms near the $f\left(z_{i}\right)$ with maps $\mathbb{C} \supset U_{i} \rightarrow \mathbb{C}^{n}$. With this identification understood, the linearized Cauchy-Riemann operator at $f$ near $z_{i}$ is given in $[\mathbf{1 9}$, Section 3.1] as

$$
D_{f} \xi=\xi_{s}+J(z) \xi_{t}+A(z) \xi
$$

with the $z$-dependent linear maps $J(z):=J(f(z))$ and $A(z):=D J(f(z))$ $\left(\cdot, f_{t}(z)\right)$. Choose the coordinate charts such that $z_{j}$ corresponds to $0 \in \mathbb{C}$ and $J(0)=i$ is the standard complex structure on $\mathbb{C}^{n}$.

Now fix $j$ as in Part (b) and let $v \in \mathbb{C}^{n}$ be given. Define the holomorphic function

$$
\tilde{\xi}(z):=z^{\ell_{j}+1} v
$$

near $z=0$ in the chart around $z_{j}$. This function satisfies $\tilde{\xi}_{s}+i \tilde{\xi}_{t}=0$ and

$$
d^{\ell_{j}} \tilde{\xi}(0)=0, \quad j^{\ell_{j}+1} \tilde{\xi}(0)=\frac{\partial^{\ell_{j}+1} \tilde{\xi}}{\partial s^{\ell_{j}+1}}(0)=\frac{\partial^{\ell_{j}+1} \tilde{\xi}}{\partial z^{\ell_{j}+1}}(0)=v .
$$


The function $\tilde{\eta}:=D_{f} \tilde{\xi}$ is given by

$$
\tilde{\eta}=\tilde{\xi}_{s}+i \tilde{\xi}_{t}+(J(z)-i) \tilde{\xi}_{t}+A(z) \tilde{\xi}=B(z) \tilde{\xi}_{t}+A(z) \tilde{\xi},
$$

where the linear map $B(z):=J(z)-i$ satisfies $B(0)=0$. Since $d^{\ell_{j}} \tilde{\xi}(0)=0$, all terms in $d^{\ell_{j}} \tilde{\eta}(0)$ vanish except possibly $B(0) j^{\ell_{j}+1} \tilde{\xi}(0)$, but this one vanishes as well because $B(0)=0$. So we have $d^{\ell_{j}} \tilde{\eta}\left(z_{j}\right)=0$.

Now extend $\tilde{\xi}$ to $S^{2}$ such that it vanishes near the $z_{i}, i \neq j$, and let $\tilde{\eta}:=D_{f} \tilde{\xi}$ on $S^{2}$. Then

$$
\begin{gathered}
d^{\ell_{j}} \tilde{\xi}\left(z_{j}\right)=0, \quad j^{\ell_{j}+1} \tilde{\xi}\left(z_{j}\right)=v \in \mathbb{C}^{n}, \\
d^{\ell_{j}+1} \tilde{\xi}\left(z_{i}\right)=0 \quad \text { for all } i \neq j, \\
d^{\ell_{j}} \tilde{\eta}\left(z_{i}\right)=0 \quad \text { for all } i=1, \ldots, k .
\end{gathered}
$$

The last equation shows that $\tilde{\eta}$ belongs to the space $E_{0}^{m-1, p}$ defined in Lemma 6.6 (with $\ell=\ell_{j}$ ), so by Lemma 6.6 there exists $\left(\xi^{\prime}, Y\right) \in B_{0}^{m, p} \oplus$ $T_{J} \mathcal{J}(V)$ such that

$$
D_{f} \xi^{\prime}+\frac{1}{2} Y(f) \circ d f \circ j=-\tilde{\eta} .
$$

It follows that the element $\left(\xi:=\xi^{\prime}+\tilde{\xi}, Y\right) \in T_{f} \mathcal{B}^{m, p} \oplus T_{J} \mathcal{J}(V)$ satisfies

$$
\begin{gathered}
D_{f} \xi+\frac{1}{2} Y(f) \circ d f \circ j=0, \\
d^{\ell_{j}} \xi\left(z_{j}\right)=0, \quad j^{\ell_{j}+1} \xi\left(z_{j}\right)=v, \\
d^{\ell_{j}+1} \xi\left(z_{i}\right)=0 \quad \text { for all } i \neq j .
\end{gathered}
$$

Now we use the hypothesis $\ell_{j} \geq \max \left\{\ell_{i}\right\}-1$, or $\ell_{i} \leq \ell_{j}+1$ for all $i \neq j$, to conclude $d^{\ell_{i}} \xi\left(z_{i}\right)=0$ for all $i=1, \ldots, k$. Hence $(\xi, Y)$ belongs to the tangent space $T_{f, J} \tilde{\mathcal{M}}_{\mathbf{z}}^{s}$ defined in $(6.1)$ and $L_{j}(\xi, Y) j_{\mathbb{C}^{n_{j}}}^{\ell_{j}+1} \xi\left(z_{j}\right)=[v] \in \mathbb{C}^{n} / \mathbb{C}^{n_{j}}$. This proves surjectivity of $L_{j}$ and hence Lemma 6.5.

Lemma 6.5 also includes the cases where some $\ell_{i}=-1$, in which case we do not impose any condition at $z_{i}$. In particular, for $m-2 / p>0$ the universal solution space

$$
\tilde{\mathcal{M}}^{s}(\mathcal{J}(V)):=\left\{(f, J) \in \mathcal{B}^{m, p} \times \mathcal{J}(V) \mid \bar{\partial}_{J} f=0, f \text { simple, } f^{-1}(V) \neq \emptyset\right\}
$$

of simple holomorphic maps meeting $V$ is a Banach manifold (this case is also covered by [19, Proposition 3.2.1]). By Lemma 6.5, for $m-2 / p>\max \left\{\ell_{i}\right\}$,

$$
\tilde{\mathcal{M}}_{\mathbf{z}}^{s}\left(\mathcal{J}(V) ;\left\{Z_{i}, \ell_{i}\right\}\right) \subset \tilde{\mathcal{M}}^{s}(\mathcal{J}(V))
$$

is a Banach submanifold that is cut out transversally by the normal jet evaluation maps $j_{Z_{i}}^{\ell} f\left(z_{i}\right), 0 \leq \ell \leq \ell_{i}$ (where $j_{Z_{i}}^{\ell} f\left(z_{i}\right)$ is defined whenever $f\left(z_{i}\right) \in Z_{i}$ and $\left.j_{Z_{i}}^{1} f\left(z_{i}\right)=\cdots=j_{Z_{i}}^{\ell-1}\left(z_{i}\right)=0\right)$. In particular, $\tilde{\mathcal{M}}_{\mathbf{z}}^{s}\left(\mathcal{J}(V) ;\left\{Z_{i}, \ell_{i}\right\}\right)$ has 
codimension

$$
2 \sum_{i=1}^{k}\left(\ell_{i}+1\right) \operatorname{codim}_{\mathbb{C}} Z_{i}
$$

in $\tilde{\mathcal{M}}^{s}(\mathcal{J}(V))$. By the Sard-Smale theorem, there exists a Baire set $\mathcal{J}^{\text {reg }}\left(V ;\left\{Z_{i}\right\}\right) \subset \mathcal{J}(V)$ such that each $J \in \mathcal{J}^{\text {reg }}\left(V ;\left\{Z_{i}\right\}\right)$ is a regular value of all the projections

$$
\pi: \tilde{\mathcal{M}}_{\mathbf{z}}^{s}\left(\mathcal{J}(V) ;\left\{Z_{i}, \ell_{i}\right\}\right) \longrightarrow \mathcal{J}(V), \quad(f, J) \longmapsto J
$$

for $\ell_{1}, \ldots, \ell_{k} \geq-1$. Here the complex submanifolds $Z_{1}, \ldots, Z_{k}$ are fixed and $\mathcal{J}^{\text {reg }}\left(V ;\left\{Z_{i}\right\}\right)$ depends on them. Now fix a homology class $A \in H_{2}(X ; \mathbb{Z})$ and define for $J \in \mathcal{J}^{\text {reg }}\left(V ;\left\{Z_{i}\right\}\right)$

$$
\begin{aligned}
& \tilde{\mathcal{M}}_{\mathbf{z}}^{s}\left(A, J ;\left\{Z_{i}, \ell_{i}\right\}\right):=\left\{f: S^{2} \longrightarrow X \mid \bar{\partial}_{J} f=0,[f]=A\right. \\
&\left.d^{\ell_{i}} f\left(z_{i}\right) \in T_{f\left(z_{i}\right)} Z_{i} \text { for } i=1, \ldots, k\right\} .
\end{aligned}
$$

By [19, Theorem 3.1.5], the space $\tilde{\mathcal{M}}^{s}(A, J)$ (corresponding to $\ell_{i}=-1$ for all $i$ ) is a smooth manifold of dimension

$$
\operatorname{dim} \tilde{\mathcal{M}}^{s}(A, J)=2 n+2 c_{1}(A) .
$$

Hence the preceding discussion shows

Lemma 6.7. Let $Z_{1}, \ldots, Z_{k}$ be $J_{0}$-complex manifolds of the $2 n$-dimensional symplectic manifold $(X, \omega)$ and $V \subset X$ an open subset with $V \cap\left(Z_{1} \cup \ldots\right.$ $\left.\cup Z_{k}\right)=\emptyset$. Then there exists a Baire set $\mathcal{J}^{\mathrm{reg}}\left(V ;\left\{Z_{i}\right\}\right) \subset \mathcal{J}(V)$ of tamed almost complex structures that agree with $J_{0}$ outside $V$ with the following property. For all integers $\ell_{1}, \ldots, \ell_{k} \geq-1$, homology classes $A \in H_{2}(X ; \mathbb{Z})$, and $J \in \mathcal{J}^{\mathrm{reg}}\left(V ;\left\{Z_{i}\right\}\right)$, the solution space $\tilde{\mathcal{M}}_{\mathbf{z}}^{s}\left(A, J ;\left\{Z_{i}, \ell_{i}\right\}\right)$ of simple $J$-holomorphic maps, passing through $V$ and tangent to $Z_{i}$ of order $\ell_{i}$ at $z_{i}$, is a smooth submanifold of $\tilde{\mathcal{M}}^{s}(A, J)$ of dimension

$$
\operatorname{dim} \tilde{\mathcal{M}}_{\mathbf{z}}^{s}\left(A, J ;\left\{Z_{i}, \ell_{i}\right\}\right)=2 n+2 c_{1}(A)-2 \sum_{i=1}^{k}\left(\ell_{i}+1\right) \operatorname{codim}_{\mathbb{C}} Z_{i} .
$$

Remark 6.8. No assumption is made on the mutual intersections of the complex submanifolds $Z_{i}$.

Lemma 6.7 deals with parametrized holomorphic curves and fixed marked points $z_{i}$. However, the vanishing of the $\ell$ th jet is a property which does not depend on the chosen complex coordinate on the Riemann surface. Hence we can define the moduli space

$$
\mathcal{M}_{k}^{s}\left(A, J ;\left\{Z_{i}, \ell_{i}\right\}\right):=\tilde{\mathcal{M}}_{k}^{s}\left(A, J ;\left\{Z_{i}, \ell_{i}\right\}\right) / \operatorname{Aut}\left(S^{2}\right)
$$

of all (unparametrized) simple $J$-holomorphic maps that pass through $V$ and are tangent to $Z_{i}$ of order $\ell_{i}$ at the (varying) marked points $z_{i}$. 
Proposition 6.9. Let $Z_{1}, \ldots, Z_{k}$ be $J_{0}$-complex submanifolds of the $2 n$ dimensional symplectic manifold $(X, \omega)$ and $V \subset X$ an open subset with $V \cap\left(Z_{1} \cup \cdots \cup Z_{k}\right)=\emptyset$. Then there exists a Baire set $\mathcal{J}^{\mathrm{reg}}\left(V ;\left\{Z_{i}\right\}\right) \subset \mathcal{J}(V)$ of tamed almost complex structures that agree with $J_{0}$ outside $V$ with the following property. For all integers $\ell_{1}, \ldots, \ell_{k} \geq-1$, homology classes $A \in H_{2}(X ; \mathbb{Z})$, and $J \in \mathcal{J}^{\mathrm{reg}}\left(V ;\left\{Z_{i}\right\}\right)$, the moduli space $\mathcal{M}_{k}^{s}\left(A, J ;\left\{Z_{i}, \ell_{i}\right\}\right)$ is a smooth manifold of dimension

$$
\operatorname{dim} \mathcal{M}_{k}^{s}\left(A, J ;\left\{Z_{i}, \ell_{i}\right\}\right)=2 n-6+2 c_{1}(A)+2 k-2 \sum_{i=1}^{k}\left(\ell_{i}+1\right) \operatorname{codim}_{\mathbb{C}} Z_{i} .
$$

Proof. (1) Consider a set $\mathcal{U}:=U_{1} \times U_{2} \times \cdots \times U_{k} \subset\left(S^{2}\right)^{k}$, where $U_{1}, \ldots, U_{k}$ are disjoint complex coordinate neighbourhoods of $S^{2}$. Let

$$
\tilde{\mathcal{M}}_{\mathcal{U}}^{s}\left(A, J ;\left\{Z_{i}, \ell_{i}\right\}\right):=\bigcup_{\mathbf{z} \in \mathcal{U}} \tilde{\mathcal{M}}_{\mathbf{z}}^{s}\left(A, J ;\left\{Z_{i}, \ell_{i}\right\}\right)
$$

be the space of simple $J$-holomorphic maps, passing through $V$ and tangent to $Z_{i}$ of order $\ell_{i}$ at $z_{i}$, where each $z_{i}$ is allowed to vary in the open set $U_{i}$. Fixing holomorphic coordinate charts on the $U_{i}$, an argument similar to the proof of Lemma 6.7 yields a Baire set $\mathcal{J}_{\mathcal{U}}^{\text {reg }}\left(V ;\left\{Z_{i}\right\}\right) \subset \mathcal{J}(V)$ such that for $J \in \mathcal{J}_{\mathcal{U}}^{\mathrm{reg}}\left(V ;\left\{Z_{i}\right\}\right)$ this is a manifold of dimension

$$
\operatorname{dim} \tilde{\mathcal{M}}_{\mathcal{U}}^{s}\left(A, J ;\left\{Z_{i}, \ell_{i}\right\}\right)=2 n+2 c_{1}(A)+2 k-2 \sum_{i=1}^{k}\left(\ell_{i}+1\right) \operatorname{codim}_{\mathbb{C}} Z_{i} .
$$

(2) Next note that we can cover the space $\tilde{\mathcal{M}}_{k}$ of $k$-tuples $\left(z_{1}, \ldots, z_{k}\right)$ of distinct points in $S^{2}$ by a countable family of such open sets $\mathcal{U}:=U_{1} \times \cdots \times U_{k}$, where $U_{1}, \ldots, U_{k} \subset S^{2}$ are disjoint coordinate neighbourhoods. Indeed, for any tuple of distinct points $\mathbf{z}=\left(z_{1}, \ldots, z_{k}\right)$ whose real and imaginary parts are rational or equal to $\infty$, let $\rho:=\min \left\{d\left(z_{i}, z_{j}\right) \mid i \neq j\right\}$ and $U_{i}$ be the open ball around $z_{i}$ of radius $\rho / 2$. Here all distances are measured with respect to the standard metric of the unit sphere. This yields a countable family of open sets $\mathcal{U}(\mathbf{z}):=U_{1} \times \cdots \times U_{k}$ of the required form. To show that this is a covering, let $\mathbf{z}=\left(z_{1}, \ldots, z_{k}\right)$ be any $k$-tuple of distinct points in $S^{2}$ and let $\rho:=\min \left\{d\left(z_{i}, z_{j}\right) \mid i \neq j\right\}$ be the minimal distance. Choose a tuple $\mathbf{z}^{\prime}=\left(z_{1}^{\prime}, \ldots, z_{k}^{\prime}\right)$ of distinct rational points such that $d\left(z_{i}, z_{i}^{\prime}\right)<\rho / 4$ for all $i$ and thus $\rho^{\prime}:=\min \left\{d\left(z_{i}^{\prime}, z_{j}^{\prime}\right) \mid i \neq j\right\}>\rho / 2$. Then we have $\mathbf{z} \in \mathcal{U}\left(\mathbf{z}^{\prime}\right)=U_{1}^{\prime} \times \cdots \times U_{k}^{\prime}$, where $U_{i}^{\prime}$ is the ball around $z_{i}^{\prime}$ of radius $\rho^{\prime} / 2>\rho / 4$.

(3) Now for each set $\mathcal{U}$ of the countable covering in part (2) let $\mathcal{J}_{\mathcal{U}}^{\text {reg }}\left(V ;\left\{Z_{i}\right\}\right) \subset \mathcal{J}(V)$ be the Baire set provided by part (1). Then the countable intersection $\mathcal{J}^{\text {reg }}\left(V ;\left\{Z_{i}\right\}\right):=\cap_{\mathcal{U}} \mathcal{J}_{\mathcal{U}}^{\text {reg }}\left(V ;\left\{Z_{i}\right\}\right)$ is again a Baire set and for $J \in \mathcal{J}^{\text {reg }}\left(V ;\left\{Z_{i}\right\}\right)$, the solution space $\tilde{\mathcal{M}}_{k}^{s}\left(A, J ;\left\{Z_{i}, \ell_{i}\right\}\right)$ is a manifold of 
dimension

$$
\operatorname{dim} \tilde{\mathcal{M}}_{k}^{s}\left(A, J ;\left\{Z_{i}, \ell_{i}\right\}\right)=2 n+2 c_{1}(A)+2 k-2 \sum_{i=1}^{k}\left(\ell_{i}+1\right) \operatorname{codim}_{\mathbb{C}} Z_{i} .
$$

Since the automorphism group $\operatorname{Aut}\left(S^{2}\right)$ acts freely and properly on this space (see [19]), the quotient $\mathcal{M}_{k}^{s}\left(A, J ;\left\{Z_{i}, \ell_{i}\right\}\right)$ is a manifold of dimension

$$
\operatorname{dim} \mathcal{M}_{k}^{s}\left(A, J ;\left\{Z_{i}, \ell_{i}\right\}\right)=2 n-6+2 c_{1}(A)+2 k-2 \sum_{i=1}^{k}\left(\ell_{i}+1\right) \operatorname{codim}_{\mathbb{C}} Z_{i}
$$

If we replace $\mathcal{J}(V)$ in Lemma 6.6 by the space $\mathcal{J}_{S^{2}}(V)$ of almost complex structures depending on points in the domain $S^{2}$, then we can drop the hypothesis that $f$ is simple. Indeed, if $f$ is constant then the hypotheses $f^{-1}(V) \neq \emptyset$ and $V \cap Z_{i}=\emptyset$ imply $\ell_{i}=-1$ for all $i$ and the result follows from Lemma 4.2. So suppose that $f$ is nonconstant. Then in the proof of Lemma 6.6, we just need to replace the cutoff construction for the section $Y$ in the target by a cutoff in the domain $S^{2} \backslash\left\{z_{1}, \ldots, z_{k}\right\}$ as in the proof of Lemma 4.1. We can also make this cutoff depend on $\mathbf{z}=\left(z_{1}, \ldots, z_{k}\right)$ and vanish outside a sufficiently small neighbourhood to ensure positivity of the pairing with the $\eta$ as in the proof of Lemma 6.6 between equations (6.3) and (6.4). As an easy instance of Lemma 3.8, we obtain a coherent section $Y$ depending on $\pi_{I}(\mathbf{z}) \in \mathcal{M}_{|I|+1}$ for any subset $I \subset\{1, \ldots, k\}$ with $|I| \geq 3$. With the modified Lemma 6.6, the proofs of Lemma 6.5 and Proposition 6.9 carry over with minor adaptations and we obtain

Proposition 6.10. Let $Z_{1}, \ldots, Z_{k}$ be $J_{0}$-complex manifolds of the $2 n$ dimensional symplectic manifold $(X, \omega), V \subset X$ an open subset with $V \cap$ $\left(Z_{1} \cup \cdots \cup Z_{k}\right)=\emptyset$, and $I \subset\{1, \ldots, k\}$ with $|I| \geq 3$. Then there exists a Baire set $\mathcal{J}_{|I|+1}^{\mathrm{reg}}\left(V ;\left\{Z_{i}\right\}\right) \subset \mathcal{J}_{|I|+1}(V)$ of coherent domain-dependent tamed almost complex structures that agree with $J_{0}$ outside $V$ with the following property. For all integers $\ell_{1}, \ldots, \ell_{k} \geq-1$, homology classes $A \in H_{2}(X ; \mathbb{Z})$, and $J \in \mathcal{J}_{|I|+1}^{\text {reg }}\left(V ;\left\{Z_{i}\right\}\right)$, the moduli space $\mathcal{M}_{k}^{*}\left(A, J ;\left\{Z_{i}, \ell_{i}\right\}\right)$ of (not necessarily simple) $J$-holomorphic maps, passing through $V$ and tangent to $Z_{i}$ of order $\ell_{i}$ at the (varying) points $z_{i}$, is a smooth submanifold of $\mathcal{M}^{*}(A, J)$ of dimension

$$
\operatorname{dim} \mathcal{M}_{k}^{*}\left(A, J ;\left\{Z_{i}, \ell_{i}\right\}\right)=2 n-6+2 c_{1}(A)+2 k-2 \sum_{i=1}^{k}\left(\ell_{i}+1\right) \operatorname{codim}_{\mathbb{C}} Z_{i} .
$$

Applying this proposition to the special points on one vertex $\alpha$ of an $I$-stable tree, we obtain

Corollary 6.11. Let $T$ be an $I$-stable $k$-labelled tree with $A_{\alpha} \neq 0$ for a unique vertex $\alpha$ (so the reduced index set $R$ labels the special points on $\alpha$ ), 
and $J_{0} \in \mathcal{J}$. Let $Z_{i}, i \in R$ be $J_{0}$-complex manifolds of $X, V \subset X$ an open subset with $V \cap Z_{i}=\emptyset$ for all $i \in R$, and $\ell_{i} \geq-1$ integers for $i \in R$. Then there exists a Baire set $\mathcal{J}_{|I|+1}^{\mathrm{reg}}(V) \subset \mathcal{J}_{|I|+1}(V)$ (depending on $T, A_{\alpha}$ and the $\left.Z_{i}, \ell_{i}\right)$ such that for all $J \in \mathcal{J}_{|I|+1}^{\text {reg }}(V)$ the moduli space $\mathcal{M}_{T}^{*}\left(A_{\alpha}, J ;\left\{Z_{i}, \ell_{i}\right\}\right)$ of (not necessarily simple) stable $J$-holomorphic maps modelled over $T$, passing through $V$ and tangent to $Z_{i}$ of order $\ell_{i}$ at the (varying) special points $z_{\alpha i}$ on the component $\alpha$, is a smooth submanifold of $\mathcal{M}_{T}^{*}\left(A_{\alpha}, J\right)$ of dimension

$$
\begin{aligned}
\operatorname{dim} \mathcal{M}_{T}^{*}\left(A_{\alpha}, J ;\left\{Z_{i}, \ell_{i}\right\}\right)= & 2 n-6+2 c_{1}\left(A_{\alpha}\right)+2 k-2 e(T) \\
& -2 \sum_{i \in R}\left(\ell_{i}+1\right) \operatorname{codim}_{\mathbb{C}} Z_{i} .
\end{aligned}
$$

As in Corollary 5.9, the dimension formula can be improved by forgetting the marked points outside $R$ and stabilizing (which yields the tree with the unique vertex $\alpha$ ).

Corollary 6.12. In the situation of Corollary 6.11, the evaluation map $\mathrm{ev}^{k}$ factors as

$$
\mathrm{ev}^{k}: \mathcal{M}_{T}^{*}\left(A_{\alpha}, J ;\left\{Z_{i}, \ell_{i}\right\}\right) \longrightarrow \mathcal{M}_{|R|}^{*}\left(A_{\alpha}, J ;\left\{Z_{i}, \ell_{i}\right\}\right) \longrightarrow X^{k}
$$

through a smooth manifold of dimension

$\operatorname{dim} \mathcal{M}_{|R|}^{*}\left(A_{\alpha}, J ;\left\{Z_{i}, \ell_{i}\right\}\right)=2 n-6+2 c_{1}\left(A_{\alpha}\right)+2|R|-2 \sum_{i \in R}\left(\ell_{i}+1\right) \operatorname{codim}_{\mathbb{C}} Z_{i}$.

\section{Intersection numbers}

A complex hypersurface is an (almost) complex submanifold $Y$ in an almost complex manifold $(X, J)$ of complex codimension one. Let $f:(\Sigma, j) \rightarrow(X, J)$ be a holomorphic map from a Riemann surface. Suppose that $f(z) \in Y$ is an isolated intersection of $f$ with $Y$. This means that there exists a closed disk $D \subset \Sigma$ around $z$ and a closed $(2 n-2)$-ball $B \subset Y$ around $f(z)$ such that $f^{-1}(B) \cap D=\{z\}$. Then the local intersection number

$$
\iota(f, Y ; z):=\left(\left.f\right|_{D}\right) \cdot B .
$$

of $f$ with $Y$ at $z$ is defined by smoothly perturbing $f$ and counting with signs (and independent of a sufficiently small perturbation). More generally, suppose that $\Sigma$ and $Y$ are compact and connected with (possibly empty) boundaries such that $f^{-1}(Y) \cap \partial \Sigma=f^{-1}(\partial Y) \cap \Sigma=\emptyset$. Then we have a welldefined intersection number

$$
\iota(f, Y):=\left(\left.f\right|_{\Sigma}\right) \cdot Y .
$$

of $f$ with $Y$. 
Proposition 7.1. Let $f:(\Sigma, j) \rightarrow(X, J)$ be holomorphic, $Y \subset X$ a complex hypersurface such that $f^{-1}(Y) \cap \partial \Sigma=f^{-1}(\partial Y) \cap \Sigma=\emptyset$ and $f(\Sigma) \not \subset Y$. Then the set $f^{-1}(Y)$ is finite and

$$
\iota(f, Y)=\sum_{z \in f^{-1}(Y)} \iota(f, Y ; z) .
$$

At each intersection point $z, f$ is tangent to $Y$ of some finite order $\ell \geq 0$ with

$$
\iota(f, Y ; z)=\ell+1 .
$$

In particular, each local intersection number $\iota(f, Y ; z)$ is positive.

Proof. We derive a local representation for $f$ in the direction normal to $Y$ near an intersection point $z$. Choose local holomorphic coordinates for $\Sigma$ near $z$ mapping $z$ to $0 \in \mathbb{C}$ and local coordinates for $X$ near $f(z) \in Y$ mapping $f(z)$ to 0 and $Y$ to $\mathbb{C}^{n-1} \times\{0\} \subset \mathbb{C}^{n}$. Moreover, we can achieve that $\{0\} \times \mathbb{C}$ are complex directions at points of $\mathbb{C}^{n-1} \times\{0\}$. Denote coordinates on $\mathbb{C}^{n}=\mathbb{C}^{n-1} \oplus \mathbb{C}$ by $x=(y, u)$. Then $J$ corresponds to an almost complex structure on $\mathbb{C}^{n}$ of the form

$$
J(x)=\left(\begin{array}{ll}
K(x) & a(x) \\
b(x) & j(x)
\end{array}\right), \quad J(y, 0)=\left(\begin{array}{cc}
K(y, 0) & 0 \\
0 & j(y, 0)
\end{array}\right),
$$

so it satisfies

$$
a(y, 0)=0, \quad b(y, 0)=0, \quad K(y, 0)^{2}=-\mathbb{1}, \quad j(y, 0)^{2}=-1 .
$$

The map $f$ corresponds to a map $f=(g, h): \mathbb{C} \supset U \rightarrow \mathbb{C}^{n}=\mathbb{C}^{n-1} \oplus \mathbb{C}$ satisfying $f_{s}+J(f) f_{t}=0$. So its last component $h: \mathbb{C} \supset U \rightarrow \mathbb{C}$ satisfies

$$
h_{s}+j(g, h) h_{t}+b(g, h) g_{t}=0 .
$$

Since $b(g, 0)=0$, we can write

$$
j(g, h)=j(g, 0)+c(g, h)(h), \quad b(g, h)=d(g, h)(h)
$$

with smooth maps

$c: \mathbb{C}^{n} \longrightarrow \operatorname{Hom}_{\mathbb{R}}\left(\mathbb{C}, \operatorname{Hom}_{\mathbb{R}}(\mathbb{C}, \mathbb{C})\right), \quad d: \mathbb{C}^{n} \longrightarrow \operatorname{Hom}_{\mathbb{R}}\left(\mathbb{C}, \operatorname{Hom}_{\mathbb{R}}\left(\mathbb{C}^{n-1}, \mathbb{C}\right)\right)$.

Then

$$
j(g, h) h_{t}=J_{0}(z) h_{t}+C(z)(h), \quad b(g, h) g_{t}=D(z)(h)
$$

with the $\mathbb{R}$-linear maps $J_{0}(z):=j(g(z), 0)$ and

$$
C(z)(\cdot):=c(g(z), h(z))(\cdot) h_{t}(z), \quad D(z)(\cdot):=d(g(z), h(z))(\cdot) g_{t}(z) .
$$

This shows that $h$ satisfies the equation

$$
h_{s}+J_{0}(z) h_{t}+A(z)(h)=0
$$

with $\mathbb{R}$-linear maps $J_{0}(z)$ and $A(z):=C(z)+D(z)$ such that $J_{0}(z)^{2}=-\mathbb{1}$ for all $z$. By the version of the Carleman similarity principle proved in $[\mathbf{1 0}]$, 
there exists a continuous matrix valued function $\Phi: \mathbb{C} \supset V \rightarrow \mathrm{GL}(2, \mathbb{R})$ and a holomorphic function $\sigma: \mathbb{C} \supset V \rightarrow \mathbb{C}$ such that

$$
h(z)=\Phi(z) \sigma(z), \quad \sigma(0)=0, \quad J_{0}(z) \Phi(z)=\Phi(z) i .
$$

Thus $h$ looks locally like the holomorphic function $\sigma$. The proposition now is an easy consequence of this representation (see [10]): If $\sigma \equiv 0$ then, by unique continuation, we would conclude $f(\Sigma) \subset Y$, which is excluded by hypothesis. Thus $\sigma(z)=z^{\ell+1} \tau(z)$ for an integer $\ell \geq 0$ and a holomorphic function $\tau$ satisfying $\tau(0) \neq 0$. This shows that 0 is an isolated intersection point of $f$ with $Y$. Moreover, $f$ is tangent to $Y$ at 0 of finite order $\ell$. Finally, the local intersection number of $f$ with $Y$ equals the local mapping degree of $h$ at 0 . Since $J_{0}(z) \Phi(z)=\Phi(z) i$, the matrix $\Phi(z)$ is orientation preserving and hence homotopic to the identity in $\operatorname{GL}(2, \mathbb{R})$. So the local mapping degree of $h$ is given by the winding number of $\sigma$, which is just $\ell+1$.

Now let $\mathbf{f}=\left(f_{\alpha}\right): \Sigma_{\mathbf{z}} \rightarrow X$ be a nonconstant genus zero nodal $J$-holomorphic curve with $k \geq 1$ marked points modelled over a tree $T$. Let $Y \subset(X, J)$ be a closed complex hypersurface. Suppose that no nonconstant component $f_{\alpha}$ is contained in $Y$. Then we define the local intersection number $\iota\left(f, Y ; z_{i}\right)$ at a marked point $z_{i}$ as follows: If $f_{\alpha_{i}}$ is nonconstant set $\iota\left(\mathbf{f}, Y ; z_{i}\right):=\iota\left(f_{\alpha_{i}}, Y ; z\right)$. If $f_{\alpha_{i}}$ is constant let $T_{1} \subset T$ be the ghost tree containing $\alpha_{i}$. Let $T_{2} \subset T \backslash T_{1}$ be the set of vertices adjacent to $T_{1}$, so $f_{\beta}$ is nonconstant for all $\beta \in T_{2}$. Recall that $z_{\beta i} \in S_{\beta}$ is the nodal point connecting $\beta$ to $T_{1}$. Then set

$$
\iota\left(\mathbf{f}, Y ; z_{i}\right):=\sum_{\beta \in T_{2}} \iota\left(f_{\beta}, Y ; z_{\beta i}\right) .
$$

Note that in this definition we allow for constant components $f_{\alpha}$ to be contained in $Y$. Also note that $\iota\left(\mathbf{f}, Y ; z_{i}\right)=\iota\left(\mathbf{f}, Y ; z_{j}\right)$ if $z_{i}$ and $z_{j}$ belong to the same ghost tree.

Lemma 7.2. Let $Y \subset(X, J)$ be a closed complex hypersurface. Let $\left(\mathbf{z}^{\nu}, \mathbf{f}^{\nu}\right)$ be a sequence of nonconstant genus zero nodal J-holomorphic maps with $k \geq 1$ marked points converging to $(\mathbf{z}, \mathbf{f})$ in the Gromov topology. Suppose that no nonconstant components of $\mathbf{f}^{\nu}$ and $\mathbf{f}$ are contained in $Y$.

(a) If $z_{1}$ lies on a nonconstant component of $\mathbf{f}$, then

$$
\iota\left(\mathbf{f}, Y ; z_{1}\right) \geq \limsup _{\nu \longrightarrow \infty} \iota\left(\mathbf{f}^{\nu}, Y ; z_{1}^{\nu}\right) .
$$

(b) If $z_{1}$ lies on a constant component of $\mathbf{f}$, let $T_{1} \subset T$ be the ghost tree containing the vertex $\alpha_{1}$. Then

$$
\iota\left(\mathbf{f}, Y ; z_{1}\right) \geq \limsup _{\nu \rightarrow \infty} \sum_{\alpha_{i} \in T_{1}} \iota\left(\mathbf{f}^{\nu}, Y ; z_{i}^{\nu}\right)
$$

where for each ghost tree $T^{\prime} \subset T^{\nu}$ at most one of the $z_{i}^{\nu}$ with $\alpha_{i} \in T^{\prime}$ is counted in the sum. 
Proof. Choose a closed ball $B$ in $X$ around $\mathbf{f}\left(z_{1}\right)$ containing no other intersection points of $Y$ with $\mathbf{f}$. Since each $\mathbf{f}^{\nu}$ intersects $Y$ in only finitely many points, we have at most countably many intersection points of $Y$ with all the $\mathbf{f}^{\nu}$. Thus we can choose $B$ such that $\partial B$ contains no intersection point of $Y$ with any of the $\mathbf{f}^{\nu}$. Moreover, we can choose $B$ such that $\partial B$ is transverse to $\mathbf{f}$ and all the $\mathbf{f}^{\nu}$ (in particular, $\partial B$ contains no nodal points of $\mathbf{f}$ or any of the $\left.\mathbf{f}^{\nu}\right)$. Let $\Sigma \subset \Sigma_{\mathbf{z}}$ be the connected component of $\mathbf{f}^{-1}(B)$ containing $z_{1}$. Similarly, let $\Sigma^{\nu} \subset \Sigma_{\mathbf{z}^{\nu}}$ be the connected component of $\left(\mathbf{f}^{\nu}\right)^{-1}(B)$ containing $z_{1}^{\nu}$. By definition of Gromov convergence, after suitable reparametrization $\left.\mathbf{f}^{\nu}\right|_{\Sigma^{\nu}}$ converges uniformly to $\left.\mathbf{f}\right|_{\Sigma}$. (Note that the domains $\Sigma^{\nu}$ and $\Sigma$ may have different numbers of nodes due to pinching, but since the maps are constant across the nodes uniform convergence makes sense.) So by definition of the intersection number,

$$
\left(\left.\mathbf{f}^{\nu}\right|_{\Sigma^{\nu}}\right) \cdot Y \longrightarrow\left(\left.\mathbf{f}\right|_{\Sigma}\right) \cdot Y
$$

as $\nu \rightarrow \infty$.

Now consider first case (a). Choosing the ball $B$ small enough, we can ensure that $z_{1}$ is the only point in $\Sigma$ with $f\left(z_{1}\right) \in Y$. This implies

$$
\left(\left.\mathbf{f}\right|_{\Sigma}\right) \cdot Y=\iota\left(\mathbf{f}, Y ; z_{1}\right) \text {. }
$$

By definition of Gromov convergence, for $\nu$ large the domain $\Sigma^{\nu}$ contains the point $z_{1}^{\nu}$. It may also contain other intersection points of $f^{\nu}$ with $Y$. But positivity of the local intersection numbers in Proposition 7.1 implies

$$
\left(\left.\mathbf{f}^{\nu}\right|_{\Sigma^{\nu}}\right) \cdot Y \geq \iota\left(\mathbf{f}^{\nu}, Y ; z_{1}^{\nu}\right)
$$

and case (a) follows.

Next consider case (b). Choosing the ball $B$ small enough, we can ensure that all points $z \in \Sigma$ with $f(z) \in Y$ belong to the tree $T_{1}$. This implies

$$
\left(\left.\mathbf{f}\right|_{\Sigma}\right) \cdot Y=\iota\left(\mathbf{f}, Y ; z_{1}\right)
$$

By definition of Gromov convergence, for $\nu$ large the domain $\Sigma^{\nu}$ contains all points $z_{i}^{\nu}$ with $\alpha_{i} \in T_{1}$. So as above, positivity of intersections implies

$$
\left(\left.\mathbf{f}^{\nu}\right|_{\Sigma^{\nu}}\right) \cdot Y \geq \sum_{\alpha_{i} \in T_{1}} \iota\left(\mathbf{f}^{\nu}, Y ; z_{i}^{\nu}\right)
$$

where the sum is to be interpreted as explained in the statement of the lemma. This proves case (b).

\section{Symplectic hypersurfaces}

Existence and asymptotic uniqueness. Let $(X, \omega)$ be a closed symplectic manifold such that $\omega$ represents an integral cohomology class $[\omega] \in H^{2}(X ; \mathbb{Z})$. By a hypersurface, we mean a closed codimension 2 submanifold $Y \subset X$ whose Poincaré dual in $H^{2}(X ; \mathbb{Z})$ equals $D[\omega]$, where $D \in \mathbb{N}$ is called the degree of $Y$. Fix a compatible almost complex structure $J$ 
on $X$. We say that a hypersurface $Y \subset X$ of degree $D$ is approximately $J$ holomorphic if its Kähler angle (see the following paragraph for the precise definition) at each point is at most $C D^{-1 / 2}$, where $C$ is a constant independent of $D$. We say that two hypersurfaces $Y, \bar{Y} \subset X$ of degrees $D, \bar{D}$ intersect $\varepsilon$-transversally if they intersect transversally and at each intersection point their tangent spaces have minimal angle (also defined in the next paragraph) at least $\varepsilon$ for an $\varepsilon>0$ independent of the degrees. The following theorem is an easy adaptation of the results in $[\mathbf{2}, \mathbf{4}]$.

Theorem 8.1 (Donaldson [4], Auroux [2]). Let $(X, \omega)$ be a closed symplectic manifold and $J$ an $\omega$-compatible almost complex structure.

(Existence). For each sufficiently large integer $D$ there exists an approximately $J$-holomorphic hypersurface $Y \subset X$ whose Poincaré dual in $H^{2}(X ; \mathbb{Z})$ equals $D[\omega]$.

(Stabilization). Let $Y \subset X$ be a J-holomorphic (not just approximately) hypersurface of degree $D$. Then there exists an $\varepsilon>0$ such that for each sufficiently large integer $\bar{D}$ there exists an approximately $J$ holomorphic hypersurface $\bar{Y}$ of degree $\bar{D}$ intersecting $Y$ E-transversally. (Uniqueness). Let $Y_{i}, i=0,1$ be two approximately $J_{i}$-holomorphic hypersurfaces arising from the construction in the (Existence) part with the same degree $D$. Connect $J_{0}, J_{1}$ by a path of $\omega$-compatible almost complex structures $J_{t}$. If $D$ is sufficiently large, then $Y_{0}, Y_{1}$ are isotopic through approximately $J_{t}$-holomorphic hypersurfaces. Moreover, this isotopy can be realized through symplectomorphisms of $X$.

Proof. (Existence) is proved in [4] and (Uniqueness) in [2]. The (Stabilization) property is not stated explicitly in the literature, but it follows from the proof in [4]. For the sake of completeness, let us indicate the required modifications.

We follow the notation of [2]. Fix a Hermitian line bundle $L \rightarrow X$ with a unitary connection $A$ of curvature $-i \omega$. For $k \in \mathbb{N}$ we consider sections $s$ in the $k$ th tensor power $L^{k}$ of $L$. We will measure all quantities with respect to the rescaled metric $g_{k}:=k g$ on $X$, where $g:=\omega(\cdot, J \cdot)$. All constants are assumed to be independent of $k$. Following [5], for positive constants $C, \eta$ we say that $s$ is $C$-bounded if

$$
\|s\|_{C^{3}}<C, \quad\|\bar{\partial} s\|_{C^{2}}<C k^{-1 / 2}
$$

and $s$ is $\eta$-transverse if $|s(x)|<\eta$ implies $\nu\left(\nabla_{x} s\right) \geq \eta$. Here $\nu\left(\nabla_{x} s\right)^{-1}$ denotes the minimal norm of a right inverse of $\nabla_{x} s$. Note that $s$ induces a section $\left.s\right|_{Y}$ in the bundle $\left.L^{k}\right|_{Y}$. We say that $s$ is $\eta$-transverse over $Y$ if $\left.s\right|_{Y}$ is $\eta$ transverse.

Claim. There exist $C, \eta>0$ and a sequence of sections $s_{k}$ of $L^{k}$ that are $C$-bounded, $\eta$-transverse, and $\eta$-transverse over $Y$. 
From this claim the (stabilization) property easily follows. As in [4], $C$-boundedness and $\eta$-transversality imply that for $k$ sufficiently large, $\bar{Y}:=s_{k}^{-1}(0)$ is a (transversally cut out) submanifold whose Kähler angle is arbitrarily small; see Lemma 8.3(b) and remark after [4, Theorem 5]. Next, $\eta$-transversality over $Y$ and $C$-boundedness imply that $\bar{Y}$ and $Y$ intersect $\varepsilon$-transversally for $\varepsilon=\eta / C$, see Lemma 8.7(a) below. Finally, $\varepsilon$-transversality of $Y$ and $\bar{Y}$ and smallness of their Kähler angles ( $Y$ has Kähler angle zero) imply that their intersection is positive and there exists a compatible almost complex structure $\bar{J}$ which is $C^{0}$-close to $J$ and makes both $Y$ and $\bar{Y}$ holomorphic; see Lemma 8.7(b) below.

To prove the claim, we follow the scheme in [4]. First recall the construction of local $C$-bounded sections $\sigma_{k, x}$ supported near a point $x \in X$. Choose a Darboux chart $\psi_{x}: \mathbb{C}^{n} \supset U \rightarrow V \subset X$ with $\psi_{x}(0)=x$ whose differential at 0 is complex linear, and a trivialization of $\psi_{x}^{*} L$ in which the connection $A$ is given by

$$
A=\frac{1}{4} \sum_{i=1}^{n}\left(z_{i} d \bar{z}_{i}-\bar{z}_{i} d z_{i}\right) .
$$

Then $f(z):=\exp \left(-k|z|^{2} / 4\right)$ defines a holomorphic section of $L^{k}$ over $U \subset \mathbb{C}^{n}$. We multiply $f$ by a cutoff function $\beta$ and transfer it to $X$ via

$$
\sigma_{k, x}:=(\beta f) \circ \tilde{\psi}^{-1}, \quad \tilde{\psi}(z):=\psi\left(k^{-1 / 2} z\right) .
$$

By choosing the $\psi_{x}$ for all $x$ with uniformly bounded $C^{3}$-norm, one can achieve that the sections $\sigma_{k, x}$ are $C$-bounded.

For $x \in Y$ we can choose $\psi_{x}$ to map the subspace $\mathbb{C}^{n-1}$ to $Y$, so the $\left.\left(\psi_{x}\right)\right|_{\mathbb{C}^{n-1}}$ define Darboux charts for $Y$ with the same properties. This implies that the $\sigma_{k, x}$ restrict to $C$-bounded sections on $Y$.

Now we globalize the construction. For each $k$ choose finitely many points $x_{i} \in X$ such that the $g_{k}$-balls $B_{1}\left(x_{i}\right)$ of radius 1 around the $x_{i}$ cover $X$. The required number of points is of order $k^{n}$, where $\operatorname{dim} X=2 n$. We can arrange that some of the points $x_{i}$ lie on $Y$ and their unit balls cover $Y$. The desired section $s$ is obtained in the form

$$
s(x)=\sum_{x_{i}} a_{k, x_{i}} \sigma_{k, x_{i}}
$$

for suitable complex numbers $a_{k, x_{i}}$. Here the coefficients $a_{k, x_{i}}$ are defined inductively to achieve $\eta_{i}$-transversality (for suitable $\eta_{i}>0$ ) on $B_{1}\left(x_{i}\right)$ without destroying it on the previous balls. The proof based on [4, Theorem 12] shows that for $x_{i} \in Y$ we can choose $a_{k, x_{i}}$ such that $\eta_{i}$-transversality holds on $B_{1}\left(x_{i}\right)$ as well as on $B_{1}\left(x_{i}\right) \cap Y$. Hence the resulting $C$-bounded section $s$ is $\eta$-transverse (with $\eta=\min \left\{\eta_{i}\right\}$ ) on $X$ as well as over $Y$. This concludes the proof of the (stabilization) property. 
Remark 8.2. We will see below that for large degrees the hypersurfaces constructed in Theorem 8.1 have the following properties.

(Existence). $\quad Y$ is symplectic and $\bar{J}$-holomorphic for a compatible almost complex structure $\bar{J}$ arbitrarily $C^{0}$-close to $J$.

(Stabilization). $\quad \bar{Y}$ is symplectic and there exists a tamed (but not necessarily compatible!) almost complex structure, $\bar{J}$, arbitrarily $C^{0}$ close to $J$ and coinciding with $J$ on $Y$, such that $Y, \bar{Y}$ are both $\bar{J}$ holomorphic.

(Uniqueness). The $Y_{t}$ are symplectic and $\bar{J}_{t}$-holomorphic for a path of compatible almost complex structures $\bar{J}_{t}$ arbitrarily $C^{0}$-close to $J_{t}$.

Linear algebra. Recall the linear algebra underlying Donaldson's construction in [4]. Consider a Hermitian vector space, i.e., a complex vector space $(V, J)$ of complex dimension $n$ with Hermitian metric

$$
(\cdot, \cdot)=\langle\cdot, \cdot\rangle-i \omega
$$

Equivalently, $(\omega, J)$ is a compatible pair of a linear symplectic and a complex structure on $V$. Define the Kähler angle $\theta(W) \in[0, \pi]$ of an oriented $\mathbb{R}$-linear subspace $W \subset V$ of real dimension $2 k$ with respect to the Hermitian structure $(\omega, J)$ by

$$
\theta(W):=\theta(W ; \omega, J):=\cos ^{-1}\left(\frac{\left.\omega^{k}\right|_{W}}{k ! \Omega_{W}}\right),
$$

where $\Omega_{W}$ is the volume form on $W$ defined by the metric and orientation. We will usually drop $\omega$ and/or $J$ in $\theta(W ; \omega, J)$ when they are clear from the context. Note that $\theta(-W)=\pi-\theta(W)$, where $-W$ is the subspace $W$ with the opposite orientation. Define the angle $\angle \in[0, \pi / 2]$ between two nonzero vectors $x, y \in V$, respectively, between a nonzero vector $y$ and an $\mathbb{R}$-linear subspace $X$, and the maximal angle between two subspaces $X, Y \subset V$ by

$$
\begin{gathered}
\angle(x, y):=\cos ^{-1}\left(\frac{|\langle x, y\rangle|}{|x||y|}\right), \quad \angle(X, y):=\inf _{0 \neq x \in X} \angle(x, y), \\
\angle M(X, Y):=\sup _{0 \neq y \in Y} \angle(X, y)=\sup _{0 \neq y \in Y} \inf _{0 \neq x \in X} \angle(x, y) .
\end{gathered}
$$

Note that $\angle_{M}(X, Y)$ depends on the order of the subspaces $X, Y$. It equals $\pi / 2$ whenever $\operatorname{dim} Y>\operatorname{dim} X$ and 0 iff $Y \subset X$. Denote by $X^{\perp}$ and $X^{\omega}$ the orthogonal, respectively, $\omega$-orthogonal complement.

\section{Lemma 8.3.}

(a) $\theta(W)=0$ iff $W$ is complex linear and the orientation of $W$ agrees with the complex orientation, $\theta(W)=\pi$ iff $W$ is complex linear and the orientation of $W$ is opposite to the complex orientation, $\theta(W)<\pi / 2$ iff $W$ is symplectic, and $\theta(W)>\pi / 2$ iff $-W$ is symplectic. 
(b) Let $A: V \rightarrow \mathbb{C}$ be $\mathbb{C}$-linear and $B: V \rightarrow \mathbb{C}$ be $\mathbb{C}$-antilinear such that $A+B: V \rightarrow \mathbb{C}$ is surjective. Then the Kähler angle of $W:=\operatorname{ker}(A+B)$ (oriented via $A+B$ ) satisfies

$$
\tan \theta(W)=\frac{2 \sqrt{|A|^{2}|B|^{2}-|(A, \bar{B})|^{2}}}{|A|^{2}-|B|^{2}} .
$$

In particular, if $|B|<|A|$ then $W$ is symplectic.

(c) For two $\mathbb{R}$-linear subspaces $X, Y \in V$,

$$
\angle_{M}(X, Y)=\angle_{M}(J X, J Y)=\angle_{M}\left(Y^{\perp}, X^{\perp}\right)=\angle_{M}\left(Y^{\omega}, X^{\omega}\right) .
$$

(d) The Kähler angle of an oriented $\mathbb{R}$-linear subspace $W$ of dimension 2 or $2 n-2$ satisfies

$$
\min \{\theta(W), \pi-\theta(W)\}=\angle_{M}(W, J W)=\angle_{M}(J W, W) .
$$

(e) The Kähler angle of an oriented $\mathbb{R}$-linear subspace $W$ of dimension 2 or $2 n-2$ satisfies

$$
\theta(W)=\theta(J W)=\theta\left(W^{\perp}\right)=\theta\left(W^{\omega}\right) .
$$

Proof. Parts (a) and (b) are proved in [4]. The first equality in (c) is clear because $J$ preserves the inner product $\langle\cdot, \cdot\rangle$, and the third equality follows from the first two because $X^{\omega}=(J X)^{\perp}$. For the second equality, note that $\angle(X, y)=\pi / 2-\angle\left(X^{\perp}, y\right)$. Using this twice, we find

$$
\begin{aligned}
\angle_{M}(X, Y) & =\sup _{y \in Y} \angle(X, y)=\sup _{y \in Y}\left(\frac{\pi}{2}-\angle\left(X^{\perp}, y\right)\right) \\
& =\frac{\pi}{2}-\inf _{y \in Y} \inf _{x^{\perp} \in X^{\perp}} \angle\left(x^{\perp}, y\right)=\frac{\pi}{2}-\inf _{x^{\perp} \in X^{\perp}} \angle\left(Y, x^{\perp}\right) \\
& =\frac{\pi}{2}-\inf _{x^{\perp} \in X^{\perp}}\left(\frac{\pi}{2}-\angle\left(Y^{\perp}, x^{\perp}\right)\right)=\sup _{x^{\perp} \in X^{\perp}} \angle\left(Y^{\perp}, x^{\perp}\right) \\
& =\angle_{M}\left(Y^{\perp}, X^{\perp}\right) .
\end{aligned}
$$

For (d) first note that for a real subspace of arbitrary dimension $\theta(W)=\pi / 2$ iff $W \cap W^{\omega}=W \cap(J W)^{\perp} \neq\{0\}$, or equivalently $J W \cap W^{\perp} \neq$ $\{0\}$. But this is equivalent to $\angle_{M}(W, J W)=\pi / 2$. For the remaining case $\theta(W) \neq \pi / 2$ we may assume, after changing the orientation on $W$ if necessary, that $W$ is symplectic, i.e., $\theta(W)<\pi / 2$.

Now assume $\operatorname{dim} W=2 n-2$. Define the complex subspace $W_{0}:=W \cap J W$. If $W_{0}=W$ all terms in the equation in (d) are 0. So assume $W_{0} \neq W$, thus $W_{0}$ has dimension $2 n-4$. Denote by $V_{1}$ its orthogonal complement in $V$ and set $W_{1}:=W \cap V_{1}$, thus $\operatorname{dim} W_{1}=2$. For $0 \neq x=x_{0}+x_{1} \in W=W_{0} \oplus W_{1}$ we have

$$
\pi_{W}(J x)=J x_{0}+\pi_{W_{1}} J x_{1},
$$


where $\pi_{W}, \pi_{W_{1}}$ are the orthogonal projections onto $W, W_{1}$. If $x_{1}=0$ we have $\pi_{W}(J x)=J x$ and thus $\angle(W, J x)=0$. If $x_{1} \neq 0$ let $y_{1} \in W_{1}$ be the unique vector such that $\left|y_{1}\right|=\left|x_{1}\right|, y_{1} \perp x_{1}$, and $\left(x_{1}, y_{1}\right)$ is an oriented basis of $W_{1}$. Since $\left\langle x_{1}, \pi_{W_{1}} J x_{1}\right\rangle=\left\langle x_{1}, J x_{1}\right\rangle=0$, we have $\pi_{W_{1}} J x_{1}=\cos \theta y_{1}$ for a unique $\theta \in[0, \pi]$. It follows that

$$
\cos \theta=\frac{\left\langle\pi_{W_{1}} J x_{1}, y_{1}\right\rangle}{\left|y_{1}\right|^{2}}=\frac{\omega\left(x_{1}, y_{1}\right)}{\left|x_{1}\right|\left|y_{1}\right|}=\cos \theta\left(W_{1}\right)=\cos \theta(W),
$$

which is positive because $W$ is symplectic. On the other hand,

$$
\left|\pi_{W} J x\right|^{2}=\left|J x_{0}+\cos \theta y_{1}\right|^{2}=\left|x_{0}\right|^{2}+\cos ^{2} \theta\left|y_{1}\right|^{2} \geq \cos ^{2} \theta|x|^{2},
$$

with equality iff $x_{0}=0$ or $\cos ^{2} \theta=1$. The latter is excluded since we assumed $W_{0} \neq W$. This shows that

$$
\cos \angle(W, J x)=\frac{\left|\pi_{W} J x\right|}{|x|} \geq \cos \theta(W),
$$

and hence $\angle(W, J x) \leq \theta(W)$, for all $x \in W$, with equality iff $x_{0}=0$. Taking the supremum over $x \in W$, this proves (d) in the case $\operatorname{dim} W=2 n-2$. The case $\operatorname{dim} W=2$ follows by an easier version of the same argument, setting $W_{0}=\{0\}$ and $W_{1}=W$. Part (e) is an immediate consequence of (c) and (d).

Remark 8.4. Property (e) of the preceding lemma can be used to derive property (b) as follows. Write

$$
A z=(z, a), \quad B z=(b, z)
$$

for vectors $a, b \in V$. Thus under the identification of $V^{*}$ with $V$ via the inner product, $A$ corresponds to $a$ and $B$ to $\bar{b}$. Surjectivity of $A+B$ is equivalent to $a \neq \lambda b$ for $\lambda \in S^{1}$. Then $W=\operatorname{ker}(A+B)$ is the orthogonal complement of the 2-dimensional oriented subspace

$$
W^{\perp}=\operatorname{span}\{a+b, J a-J b\} .
$$

To determine $\theta:=\theta(W)=\theta\left(W^{\perp}\right)$, we compute

$$
\begin{gathered}
\omega(a+b, J a-J b)=|a|^{2}-|b|^{2}, \\
|a+b|^{2}|J a-J b|^{2}-\langle a+b, J a-J b\rangle^{2}=\left(|a|^{2}+|b|^{2}\right)^{2}-4|(a, b)|^{2} .
\end{gathered}
$$

If $|a|=|b|$ this shows that $\theta(W)=\pi / 2$. If $|a| \neq|b|$ we conclude (b) from

$$
\begin{gathered}
\cos \theta=\frac{|a|^{2}-|b|^{2}}{\sqrt{\left(|a|^{2}+|b|^{2}\right)^{2}-4|(a, b)|^{2}}}, \quad \sin \theta=\frac{2 \sqrt{|a|^{2}|b|^{2}-|(a, b)|^{2}}}{\sqrt{\left(|a|^{2}+|b|^{2}\right)^{2}-4|(a, b)|^{2}}}, \\
\tan \theta=\frac{2 \sqrt{|a|^{2}|b|^{2}-|(a, b)|^{2}}}{|a|^{2}-|b|^{2}} .
\end{gathered}
$$


Let $(V, J, \omega)$ be a Hermitian vector space as above. To a nonzero skewsymmetric bilinear form $\sigma$ on $V$ we associate the quantities

$$
\begin{aligned}
\alpha(\sigma) & :=\inf _{x \in V, \sigma(x, J x)>0} \frac{\sigma(x, J x)}{|x|^{2}}-\inf _{x \in V, \sigma(x,-J x)>0} \frac{\sigma(x,-J x)}{|x|^{2}}, \\
\beta(\sigma) & :=\sup _{\operatorname{dim} W=2} \frac{\left.\sigma\right|_{W}}{\Omega_{W}}=\sup _{x, y} \frac{\sigma(x, y)}{\sqrt{|x|^{2}|y|^{2}-\langle x, y\rangle^{2}}}, \\
\gamma(\sigma) & :=\frac{\alpha(\sigma)}{\beta(\sigma)},
\end{aligned}
$$

where the infima are interpreted as zero over the empty set, the first supremum is taken over all oriented 2-planes $W \subset V$, and the second one over all ordered pairs of linearly independent vectors $x, y \in V$. Note that $\alpha, \beta, \gamma$ depend on $J$ and the Hermitian metric. The following properties follow directly from the definitions.

(i) For each $\sigma$, at most one of the two terms in the definition of $\alpha(\sigma)$ is nonzero. More precisely, the following three cases can occur. If $\sigma(x, J x) \geq 0$ for all $x$ the second term vanishes; if $\sigma(x, J x) \leq 0$ for all $x$ the first term vanishes; and in the remaining case both terms vanish (for if $\sigma(x, J x)>0$ and $\sigma(y, J y)<0$, then $\sigma(x+t y, J(x+t y))=0$ for some $t>0)$.

(ii) $\beta(\sigma)>0$ for every nonzero $\sigma$ and $|\alpha(\sigma)| \leq \beta(\sigma)$ (just take $y=J x$ in the definition of $\beta$ ), thus $|\gamma(\sigma)| \leq 1$. Equality $\gamma(\sigma)= \pm 1$ holds iff $\sigma= \pm c \omega$ for some $c>0$.

(iii) $\gamma(\sigma)>0$ iff $\sigma$ is a symplectic form taming $J$ and $\gamma(\sigma)<0$ iff $\sigma$ is a symplectic form taming $-J$.

(iv) $\gamma(t \sigma)=\gamma(\sigma)$ for every $t>0$ and $\gamma(-\sigma)=-\gamma(\sigma)$. Moreover, $\gamma(\sigma+$ $\left.\sigma^{\prime}\right) \geq \min \left\{\gamma(\sigma), \gamma\left(\sigma^{\prime}\right)\right\}$ if $\gamma(\sigma)$ and $\gamma\left(\sigma^{\prime}\right)$ are both positive.

Here the last property follows from $\beta\left(\sigma+\sigma^{\prime}\right) \leq \beta(\sigma)+\beta\left(\sigma^{\prime}\right)$ and $\alpha(\sigma+$ $\left.\sigma^{\prime}\right) \geq \alpha(\sigma)+\alpha\left(\sigma^{\prime}\right) \geq \min \left\{\gamma(\sigma), \gamma\left(\sigma^{\prime}\right)\right\}\left[\beta(\sigma)+\beta\left(\sigma^{\prime}\right)\right]$. In view of $\gamma( \pm \omega)= \pm 1$, $\cos ^{-1}(\gamma(\sigma)) \in[0, \pi]$ can be interpreted as the "angle" between $\omega$ and $\sigma$.

\section{Lemma 8.5.}

(a) If $\sigma$ is a symplectic form on $V$ and $W \subset V$ a codimension 2 $\omega$-symplectic subspace whose Kähler angle $\theta(W)$ (with respect to $\omega$ ) satisfies

$$
\theta(W)<\gamma(\sigma)
$$

then $W$ and $W^{\perp}$ are $\sigma$-symplectic.

(b) If $\sigma$ is a symplectic form taming $J$ and $K: V \rightarrow V$ a complex structure such that

$$
\|K-J\|<\gamma(\sigma),
$$

then $\sigma$ tames $K$. 
(c) For every codimension two $\omega$-symplectic subspace $W \subset V$ there exists an $\omega$-compatible complex structure $K: V \rightarrow V$ which leaves $W$ and $W^{\omega}$ invariant and satisfies

$$
\|K-J\| \leq \theta(W) .
$$

Remark 8.6. In fact, in part (a) the actual hypothesis is $\sin \theta(W)<\gamma(\sigma)$ and in (c) the actual conclusion is $\|K-J\| \leq 2 \sin (\theta(W) / 2)$.

Proof. (a) For $0 \neq x \in W$ write

$$
J x=\pi_{W}(J x)+y
$$

with $y \perp W$. By Lemma 8.3(d) we have $\angle(W, J x) \leq \theta(W)$, and hence $\left|\pi_{W}(J x)\right| \geq|x| \cos \theta(W)$, or equivalently, $|y| \leq|x| \sin \theta(W)$. Then

$$
\begin{aligned}
\sigma\left(x, \pi_{W}(J x)\right) & =\sigma(x, J x)-\sigma(x, y) \\
& \geq \alpha(\sigma)|x|^{2}-\beta(\sigma)|x||y| \\
& \geq|x|^{2}(\alpha(\sigma)-\beta(\sigma) \sin \theta(W)) \\
& >0
\end{aligned}
$$

by hypothesis and $\sin \theta \leq \theta$. Since $\pi_{W}(J x) \in W$, this proves that $W$ is $\sigma$ symplectic. The statement for $W^{\perp}$ follows by the same argument because $\theta\left(W^{\perp}\right)=\theta(W)$ by Lemma $8.3(\mathrm{e})$.

(b) By hypothesis, we find for every $0 \neq x \in V$ :

$$
\begin{aligned}
\sigma(x, K x) & =\sigma(x, J x)+\sigma(x,(K-J) x) \geq \alpha(\sigma)|x|^{2}-\beta(\sigma)\|K-J\||x|^{2} \\
& =\beta(\sigma)(\gamma(\sigma)-\|K-J\|)|x|^{2}>0 .
\end{aligned}
$$

(c) Let $W_{0}:=W \cap J W$. If $\operatorname{dim} W_{0}=2 n-2$, then $W$ is $J$-complex and we simply set $K:=J$. If $\operatorname{dim} W_{0}=2 n-4$, we choose $K$ such that $K=J$ on $W_{0}$ and $K$ leaves $W_{0}^{\omega}=W_{0}^{\perp}$ invariant. After replacing $V$ by $W_{0}^{\perp}$, it thus remains to consider the case $\operatorname{dim} V=4$.

For $\operatorname{dim} V=4$ choose an oriented orthonormal basis $x, y$ of $W$ and denote by $\pi_{W}: V \rightarrow W$ the orthogonal projection. Since $\langle J x, x\rangle=0$, we have $\pi_{W} J x=\cos \theta y$ for some $\theta \in[0, \pi]$. Now $\cos \theta=\langle J x, y\rangle=\omega(x, y)>0$ implies $\theta \leq \pi / 2$, and it follows from Lemma 8.3(d) that $\theta \leq \theta(W)$. Define $K: W \rightarrow$ $W$ as positive rotation by 90 degrees, i.e., $K x:=y$ and $K y:=-x$. Then clearly $K^{2}=-11$. Moreover,

$$
|J y-K y|^{2}=|J x-K x|^{2}=|J x-y|^{2}=2-2 \cos \theta=4 \sin ^{2} \frac{\theta}{2}
$$

and $\theta \leq \theta(W)$ shows $\|K-J\| \leq 2 \sin (\theta(W) / 2)$. Finally, $\omega(x, K x)=\omega(x, y)>$ 0 shows that $\omega$ tames $K$ and $K$-invariance of $\omega$ is automatic because $W$ is 2-dimensional, so $K: W \rightarrow W$ is compatible with $\omega$. Defining $K: W^{\omega} \rightarrow$ $W^{\omega}$ in the analogous way we obtained the desired $K: V \rightarrow V$. This concludes the proof of Lemma 8.5. 
For an $\mathbb{R}$-linear map $T: V \rightarrow V^{\prime}$ between Euclidean vector spaces define $\nu(T) \geq 0$ as follows. If $T$ is not surjective set $\nu(T):=0$; otherwise let $\nu(T)^{-1}$ be the minimal norm of a right inverse of $T$. Define the minimal angle between two subspaces $X, Y \subset V$ of a Euclidean vector space as follows: If $X$ and $Y$ are not transverse set $\angle_{m}(X, Y):=0$; otherwise let $X^{\prime}, Y^{\prime}$ be the orthogonal complements of $X \cap Y$ in $X, Y$ and set

$$
\angle_{m}(X, Y):=\inf _{0 \neq y \in Y^{\prime}} \angle(X, y)=\inf _{0 \neq x \in X^{\prime}, 0 \neq y \in Y^{\prime}} \angle(x, y) .
$$

\section{Lemma 8.7.}

(a) Let $T: V \rightarrow V^{\prime}$ be a nonzero $\mathbb{R}$-linear map between Euclidean vector spaces and let $W \subset V$ be a linear subspace. Then

$$
\angle_{m}(W, \operatorname{ker} T) \geq \frac{\nu\left(\left.T\right|_{W}\right)}{\|T\|} .
$$

(b) For every $\varepsilon>0$ and $\theta_{2}>0$ there exists a $\theta_{3}>0$ with the following property. For any pair of codimension two $\omega$-symplectic subspaces $W, W^{\prime}$ of a Hermitian vector space $(V, \omega, J)$ with $\angle_{m}\left(W, W^{\prime}\right) \geq \varepsilon$ and $\max \left\{\theta(W), \theta\left(W^{\prime}\right)\right\} \leq \theta_{3}$ there exists an $\omega$-tamed complex structure $K: V \rightarrow V$ which leaves $W$ and $W^{\prime}$ invariant and satisfies

$$
\|K-J\|<\theta_{2} .
$$

In particular, $W$ and $W^{\prime}$ intersect positively.

Proof. (a) Suppose that $\left.T\right|_{W}$ is surjective (otherwise both sides are zero). Set $\eta:=\nu\left(\left.T\right|_{W}\right)$ and let $W^{\prime} \subset W$ be the orthogonal complement of $W \cap \operatorname{ker} T$. Consider $w \in W^{\prime}$ and $v \in \operatorname{ker} T$ with $|v|=|w|=1$. After possibly replacing $v$ by $-v$, we may assume $\langle v, w\rangle \geq 0$. The definition of $\nu\left(\left.T\right|_{W}\right)$ implies $|T w| \geq \eta$, and therefore

$$
\eta^{2} \leq|T v-T w|^{2} \leq\|T\|^{2}|v-w|^{2}=2\|T\|^{2}(1-|\langle v, w\rangle|) .
$$

So the angle $\theta:=\angle(v, w) \in[0, \pi / 2]$ satisfies

$$
1-\frac{\eta^{2}}{2\|T\|^{2}} \geq \cos \theta \geq 1-\frac{\theta^{2}}{2}
$$

thus $\theta \geq \eta /\|T\|$ and the part (a) follows.

(b) We construct $K$ by constructing a $K$-complex basis $x_{1}, y_{1}=K x_{1}$, $\ldots, x_{n}, y_{n}=K x_{n}$ of $V$ such that $x_{1}, \ldots, y_{n-2}$ is a basis of $Z:=W \cap W^{\prime}$, $x_{1}, \ldots, y_{n-1}$ is a basis of $W$, and $x_{1}, \ldots, y_{n-2}, x_{n}, y_{n}$ is a basis of $W^{\prime}$. Choose any unit vector $x_{1} \in W \cap W^{\prime}$ and let $y_{1}:=\pi_{Z} J x_{1}$, where $\pi_{Z}$ denotes the orthogonal projection onto $Z$. Then Lemma $8.3(\mathrm{~d})$ implies $\angle\left(y_{1}, J x_{1}\right)=$ 
$\angle\left(Z, J x_{1}\right) \leq \theta_{3}<\pi / 2$ and $\left|y_{1}-J x_{1}\right| \leq \sin \theta_{3} \leq \theta_{3}$. Since $y_{1} \neq 0$, we can define a complex structure $K$ on $Z_{1}:=\operatorname{span}\left\{x_{1}, y_{1}\right\}$ by $K x_{1}:=y_{1}$, $K y_{1}:=-x_{1}$ and it satisfies $|K v-J v| \leq \theta_{3}|v|$ for all $v \in Z_{1}$. Intersecting $V, W, W^{\prime}$ with $Z_{1}^{\perp}$ and repeating this process, we construct a complex structure $K$ on $Z$ together with a $K$-complex basis $x_{1}, \ldots, y_{n-2}$ such that $|K v-J v| \leq \theta_{3}|v|$ for all $v \in Z$. Finally, we apply the same process to $W \cap Z^{\perp}$ and $W^{\prime} \cap Z^{\perp}$ in $Z^{\perp}$ to construct a complex structure $K$ on $V$ together with the basis elements $y_{n-1}=K x_{n-1} \in W$ and $y_{n}=K x_{n} \in W^{\prime}$ such that $\|K v-J v\| \leq \theta_{3}|v|$ for all $v \in W \cup W^{\prime}$. Note that this does not imply $\|K-J\| \leq \theta_{3}$ since $W, W^{\prime}$ need not be perpendicular. It does imply, however, that $\|K-J\|<\theta_{2}$ if $\angle_{m}\left(W, W^{\prime}\right) \geq \varepsilon$ for fixed $\varepsilon>0$ and $\theta_{3}$ is sufficiently small. For this we have to check that under these conditions $|(K-J) v| \leq \theta_{2}|v|$ for all vectors $v$. Note that this is satisfied for $v \in W$ or $v \in W^{\prime}$. Hence it is sufficient to check it for $v \in Z^{\perp}$ and the problem is reduced to $\operatorname{dim}_{\mathbb{R}} V=4, Z=0$. Let $W=\operatorname{span}\left\{x_{1}, y_{1}\right\}$ and $W^{\prime}=\operatorname{span}\left\{x_{2}, y_{2}\right\}$. Choose $v=a x_{1}+b y_{1}+c x_{2}+d y_{2}$. Then

$$
(K-J) v=a\left(y_{1}-J x_{1}\right)-b J\left(y_{1}-J x_{1}\right)+c\left(y_{2}-J x_{2}\right)-d J\left(y_{2}-J x_{2}\right) .
$$

On the other hand, for $w \in W$ and $w^{\prime} \in W^{\prime}$ we have $\left\langle w, w^{\prime}\right\rangle \leq \cos \varepsilon|w|\left|w^{\prime}\right|$. Hence we get

$$
\begin{aligned}
|(K-J) v|^{2} \leq & \left(a^{2}+b^{2}\right)\left|y_{1}-J x_{1}\right|^{2}+\left(c^{2}+d^{2}\right)\left|y_{2}-J x_{2}\right|^{2} \\
& +2 \cos \varepsilon \sqrt{\left(a^{2}+b^{2}\right)\left|y_{1}-J x_{1}\right|^{2}} \sqrt{\left(c^{2}+d^{2}\right)\left|y_{2}-J x_{2}\right|^{2}}
\end{aligned}
$$

and

$$
|v|^{2} \geq a^{2}+b^{2}\left|y_{1}\right|^{2}+c^{2}+d^{2}\left|y_{2}\right|^{2}-2 \cos \varepsilon \sqrt{a^{2}+b^{2}\left|y_{1}\right|^{2}} \sqrt{c^{2}+d^{2}\left|y_{2}\right|^{2}} .
$$

Since $\left|y_{i}-J x_{i}\right| \leq \sin \theta_{3}$ the first inequality leads to

$$
\begin{aligned}
|(K-J) v|^{2} & \leq\left(a^{2}+b^{2}+c^{2}+d^{2}+2 \cos \varepsilon \sqrt{a^{2}+b^{2}} \sqrt{c^{2}+d^{2}}\right) \sin ^{2} \theta_{3} \\
& \leq(1+\cos \varepsilon) \sin ^{2} \theta_{3}\left(a^{2}+b^{2}+c^{2}+d^{2}\right)
\end{aligned}
$$

and since $\left|y_{i}\right| \geq \cos \theta_{3}$ the second inequality leads to

$$
|v|^{2} \geq(1-\cos \varepsilon) \cos ^{2} \theta_{3}\left(a^{2}+b^{2}+c^{2}+d^{2}\right)
$$

from which the statement follows.

Lemma 8.5(b) (with $\sigma=\omega$ ) shows that $K$ is $\omega$-tamed if $\theta_{2}<1$.

Remark 8.8. In Lemma 8.7(b) the complex structure $K$ can, in general, not be chosen to be $\omega$-compatible. For an explicit counterexample, consider a 4-dimensional $(V, \omega, J)$ with symplectic basis $e_{1}, f_{1}=J e_{1}, e_{2}, f_{2}=J e_{2}$. The subspaces $W=\operatorname{span}\left\{e_{1}, f_{1}\right\}$ and $W^{\prime}=\operatorname{span}\left\{e_{2}+\varepsilon e_{1}, f_{2}\right\}$ are approximately $J$-complex and mutually transverse for $\varepsilon$ small, but for $\varepsilon \neq 0$ they are not invariant under a compatible complex structure $K$. Indeed, the linear form 
$i_{e_{1}} \omega$ vanishes on $W^{\prime}$, and so does $i_{K e_{1}} \omega$ for any compatible $K$ leaving $W^{\prime}$ invariant. If $K$ preserved also $W$ this would imply vanishing of $i_{f_{1}} \omega$ on $W^{\prime}$, which is not the case for $\varepsilon \neq 0$.

Recall from [18] that any pair of $\omega$-tamed complex structures $J_{0}, J_{1}$ can be connected by a canonical path $J_{t}$ of $\omega$-tamed complex structures, and if $J_{0}, J_{1}$ are $\omega$-compatible the $J_{t}$ are $\omega$-compatible as well. Moreover, the construction in $[\mathbf{1 8}]$ shows

Lemma 8.9. For every $\theta_{0}>0$ there exist $\theta_{1}>\theta_{2}>0$ such that $\left\|J_{1}-J_{0}\right\|<$ $\theta_{1}$ implies $\left\|J_{t}-J_{0}\right\|<\theta_{0}$ for all $t \in[0,1]$, and $\left\|J_{1}-J_{0}\right\|<\theta_{2}$ implies $\left\|J_{t}-J_{0}\right\|<\theta_{1}$ for all $t \in[0,1]$.

A priori estimates. Consider now a closed symplectic manifold $(X, \omega)$ with a fixed compatible almost complex structure $J$. For a codimension 2 submanifold $Y \subset X$ define

$$
\theta(Y ; \omega, J):=\sup _{y \in Y} \theta\left(T_{y} Y ; \omega, J\right),
$$

where $\theta\left(T_{y} Y ; \omega, J\right)$ is the Kähler angle with respect to the Hermitian structure $(\omega, J)$ on $T X$. Denote by $\mathcal{J}(X)$ the space of $\omega$-tamed almost complex structures on $X$. Fix constants

$$
0<\theta_{2}<\theta_{1}<\theta_{0}<1
$$

as in Lemma 8.9.

Now assume that $\omega$ represents an integral cohomology class $[\omega] \in H^{2}(X ; \mathbb{Z})$. By Theorem 8.1, for every sufficiently large integer $D>0$ there exists a closed submanifold $Y \subset X$ of codimension 2 which is Poincaré dual to $D[\omega]$ and approximately $J$-holomorphic in the sense that

$$
\theta(Y ; \omega, J)<\theta_{2} \text {. }
$$

In view of the hypothesis $\theta_{2}<1<\pi / 2, Y$ is symplectic for $\omega$.

By Lemma $8.5(\mathrm{c})$ there exists an $\omega$-compatible almost complex structure $K$ on the vector bundle $\left.T X\right|_{Y}$ with

$$
\|K-J\|_{Y}<\theta_{2}, \quad K(T Y)=T Y, \quad K\left(T Y^{\omega}\right)=T Y^{\omega} .
$$

By Lemma 8.9 and the choice of $\theta_{2}<\theta_{1}$, we can extend $K$ to an $\omega$-compatible almost complex structure on $X$ satisfying

$$
\|K-J\|<\theta_{1} .
$$

Indeed, first extend $K$ to some $K_{1}$ on $\{x \in X \mid \operatorname{dist}(x, Y) \leq r\}$ in the metric $\omega(., J$.$) such that \left\|K_{1}-J\right\|<\theta_{2}$, which is possible because this is an open condition. By the lemma there is a path $K_{t}$ on that tube connecting $K_{1}$ to $K_{0}=J$ and satisfying $\left\|K_{t}-J\right\|<\theta_{1}$ for all $t$. Finally, choose a smooth function $\phi:[0, r] \rightarrow 1$ which is equal to 0 near $r$ and equal to 1 near 0 and 
set $K_{x}:=K_{\phi(\operatorname{dist}(x, Y))}$ for $x \in X$. The upshot of this discussion is that the space

$$
\mathcal{J}(X, Y):=\{K \in \mathcal{J}(X) \mid K(T Y)=T Y\}
$$

contains a nonempty open subspace

$$
\mathcal{J}\left(X, Y ; J, \theta_{1}\right):=\left\{K \in \mathcal{J}(X, Y) \mid\|K-J\|<\theta_{1}\right\} .
$$

Moreover, by the preceding argument and Lemma 8.9, any two elements in $\mathcal{J}\left(X, Y ; J, \theta_{1}\right)$ can be connected by a path in

$$
\mathcal{J}\left(X, Y ; J, \theta_{0}\right):=\left\{K \in \mathcal{J}(X, Y) \mid\|K-J\|<\theta_{0}\right\} .
$$

Remark 8.10. The spaces $\mathcal{J}(X, Y)$ and $\mathcal{J}\left(X, Y ; J, \theta_{i}\right)$ consist of $\omega$-tame almost complex structures, but the preceding argument shows that the corresponding spaces of $\omega$-compatible almost complex structures are also nonempty.

The following lemma and proposition follow a suggestion of D. Auroux. Fix a closed 2-form $\alpha$ representing the first Chern class $c_{1}=c_{1}(T X)$ and denote by $\|\alpha\|$ its norm with respect to the metric $\omega(\cdot, J \cdot)$.

Lemma 8.11. Let $K$ be an $\omega$-tamed almost complex structure with $\| K-$ $J \|<\theta_{0}$. Then for every homology class $A \in H_{2}(X ; \mathbb{Z})$ which contains a closed $K$-holomorphic curve we have

$$
\left\langle c_{1}(T X), A\right\rangle \leq D_{*} \omega(A) \quad \text { with } \quad D_{*}=D_{*}(X, \omega, J):=\frac{1+\theta_{0}}{1-\theta_{0}}\|\alpha\| .
$$

Proof. For every tangent vector $v \in T_{x} X$, we have $\|v\|^{2}=\omega_{0}(v, J v)$ and the estimates

$$
\alpha(v, K v) \leq\|\alpha\|(1+\|K-J\|)\|v\|^{2}, \quad \omega(v, K v) \geq(1-\|K-J\|)\|v\|^{2},
$$

which combine with $\|K-J\|<\theta_{0}$ to

$$
\alpha(v, K v) \leq D_{*} \omega(v, K v) .
$$

For a closed $K$-holomorphic curve $f: \Sigma \rightarrow X$ in the class $A$ this implies

$$
\left\langle c_{1}(T X), A\right\rangle=\int_{\Sigma} f^{*} \alpha \leq D_{*} \int_{\Sigma} f^{*} \omega=D_{*} \omega(A) .
$$

Remark 8.12. For a given cohomology class $c \in H^{2}(X, \mathbb{R})$, one may wonder how much the quantity

$$
\|c\|_{g_{J}}:=\inf \left\{\|\alpha\|_{g_{J}} \mid d \alpha=0,[\alpha]=c\right\}
$$

with respect to the metric $g_{J}=\omega(\cdot, J \cdot)$ depends on the compatible almost complex structure $J$. For example, the quantity

$$
\sup _{J}\|c\|_{g_{J}}
$$

equals 1 for $c=[\omega]$. Is it finite for $c=c_{1}(X)$ ? 
In the following the energy of a $K$-holomorphic sphere $f: S^{2} \rightarrow X$ will always be measured with respect to $\omega$, i.e., $E(f)=\int_{S^{2}} f^{*} \omega$.

Proposition 8.13. Let $Y \subset X$ be a hypersurface Poincaré dual to $D[\omega]$ with $\theta(Y ; \omega, J)<\theta_{2}$ and let $K \in \mathcal{J}\left(X, Y ; J, \theta_{0}\right)$. Let $E>0$ and suppose:

(i) All moduli spaces of simple $K$-holomorphic spheres in $Y$ of energy at most $E$ are smooth manifolds of the expected dimension.

(ii) All moduli spaces of nonconstant simple $K$-holomorphic spheres of energy at most $E$ in $X$ with prescribed tangency of order $\ell \leq D_{*} E+n$ to $Y$ are smooth manifolds of the expected dimension.

Then the following holds.

(a) If $D>\max \left(D_{*}, D_{*}+n-4\right)$, then all $K$-holomorphic spheres of energy at most $E$ contained in $Y$ are constant.

(b) If $D>2 \max \left(D_{*}, D_{*}+n-2\right)$, then every nonconstant $K$-holomorphic sphere of energy at most $E$ in $X$ intersects $Y$ in at least 3 distinct points in the domain.

Proof. (a) Suppose $f$ is a nonconstant $K$-holomorphic sphere of energy at most $E$ contained in $Y$. After replacing $f$ by its underlying simple curve, we may assume that $f$ is simple. Let $A \in H_{2}(Y ; \mathbb{Z})$ be its homology class. The expected dimension of the moduli space of simple $K$-holomorphic spheres in $Y$ in the homology class $A$ equals

$$
\operatorname{ind}(A)=2(n-1)-6+2\left\langle c_{1}(T Y), A\right\rangle .
$$

Now note that

$$
c_{1}(T Y)=c_{1}\left(\left.T X\right|_{Y}\right)-c_{1}(N(Y, X))
$$

and the first Chern class of the normal bundle $N(Y, X)$ is represented by $\left.D \omega\right|_{Y}$. In view of Lemma 8.11, this implies

$$
\begin{aligned}
\operatorname{ind}(A) & =2 n-8+2\left\langle c_{1}(T X), A\right\rangle-2 D \omega(A) \\
& \leq 2 n-8+2\left(D_{*}-D\right) \omega(A) .
\end{aligned}
$$

Now $\omega(A) \geq 1$ due to the integrality of $[\omega]$ and the assumption that $f$ is nonconstant. Hence ind $(A)<0$ if $D>\max \left(D_{*}, D_{*}+n-4\right)$, so by hypothesis

(i) $f$ cannot exist.

(b) Suppose $f$ is a nonconstant $K$-holomorphic sphere of energy at most $E$ in $X$ which intersects $Y$ in at most 2 distinct points in the domain. Again after replacing $f$ by its underlying simple curve, we may assume that $f$ is simple. Let $A \in H_{2}(Y ; \mathbb{Z})$ be its homology class. For $I \leq D_{*} E+n+1$ let $\tilde{\mathcal{M}}^{s}(Y, I ; A, K)$ be the space of all simple $K$-holomorphic spheres in the 
class $A$ that have local intersection number at least $I$ (i.e., a tangency of order $\geq I-1$ ) with $Y$ at one point. By hypothesis (ii) and Proposition 6.9 $\tilde{\mathcal{M}}^{*}(Y, I ; A, K)$ is a smooth manifold of dimension

$$
\operatorname{dim} \tilde{\mathcal{M}}^{*}(Y, I ; A, K)=2 n-4+2 c_{1}(A)-2 I \geq 0 .
$$

Hence the local intersection number of $f$ with $Y$ at each point satisfies

$$
I \leq c_{1}(A)+n-2 \leq D_{*} \omega(A)+n-2 .
$$

On the other hand, if $D>2 \max \left(D_{*}, D_{*}+n-2\right)$, then the global intersection number of $f$ with $Y$ satisfies

$$
[f] \cdot[Y]=D \omega(A)>2\left(D_{*} \omega(A)+n-2\right) \geq 2 I
$$

(where we have used again $\omega(A) \geq 1$ ). Hence $f$ must have at least 3 intersection points with $Y$, which contradicts our assumption.

We will also need the following version of the previous proposition for families of almost complex structures. As above let $Y \subset X$ be a hypersurface Poincaré dual to $D[\omega]$ with $\theta(Y ; \omega, J)<\theta_{2}$. Let $\mathcal{K} \subset \mathcal{J}\left(X, Y ; J, \theta_{0}\right)$ be a family of almost complex structures smoothly depending on a parameter $\tau \in P$ varying in a $k$-dimensional manifold $P$. Moduli spaces of $\mathcal{K}$-holomorphic spheres are spaces of pairs $(u, \tau)$, where $\tau \in P$ and $u$ is a $\mathcal{K}_{\tau}$-holomorphic sphere (possibly with constraints). The corresponding transformation groups act on $u$ only. The (expected) dimension of the moduli spaces is increased by $k$ compared to the expected dimension of the corresponding moduli spaces with fixed almost complex structure. The proof of the following proposition is literally the same as the one of Proposition 8.13, each time adding $k$ to the term representing the dimension of the moduli space.

Proposition 8.14. Let $E>0$ and suppose:

(i) All moduli spaces of simple $\mathcal{K}$-holomorphic spheres in $Y$ of energy at most $E$ are smooth manifolds of the expected dimension.

(ii) All moduli spaces of nonconstant simple $\mathcal{K}$-holomorphic spheres of energy at most $E$ in $X$ with prescribed tangency of order $\ell \leq D_{*} E+$ $n+k$ to $Y$ are smooth manifolds of the expected dimension.

Then the following holds.

(a) If $2 D>\max \left(2 D_{*}, 2 D_{*}+2 n-8+k\right)$, then all $\mathcal{K}$-holomorphic spheres of energy at most $E$ contained in $Y$ are constant.

(b) If $D>2 \max \left(D_{*}, D_{*}+n-2\right)+k$, then every nonconstant $\mathcal{K}$-holomorphic sphere of energy at most $E$ in $X$ intersects $Y$ in at least 3 distinct points in the domain. 
Definition 8.15. For $(J, Y)$ as above and $E>0$ let

$$
\mathcal{J}^{*}\left(X, Y ; J, \theta_{1}, E\right) \subset \mathcal{J}\left(X, Y ; J, \theta_{1}\right)
$$

be the subset of those $K$ for which the conclusions (a) and (b) of Proposition 8.13 hold, i.e.,

(a) all $K$-holomorphic spheres of energy at most $E$ contained in $Y$ are constant.

(b) every nonconstant $K$-holomorphic sphere of energy at most $E$ in $X$ intersects $Y$ in at least 3 distinct points in the domain.

Define

$$
D^{*}:=D^{*}(W, \omega, J):=2\left(D_{*}+n\right) .
$$

Note that each degree $D \geq D^{*}$ satisfies the conditions in (a) and (b) of Proposition 8.13.

Corollary 8.16. Let $(J, Y)$ be as in Proposition 8.13 and suppose $D \geq D^{*}$ $(X, \omega, J)$. Then $\mathcal{J}^{*}\left(X, Y ; J, \theta_{1}, E\right)$ is open and dense in $\mathcal{J}\left(X, Y ; J, \theta_{1}\right)$ for every $E>0$. Moreover, for $\theta_{2}<\theta_{1}$ any two elements in $\mathcal{J}^{*}\left(X, Y ; J, \theta_{2}, E\right)$ can be connected by a path in $\mathcal{J}^{*}\left(X, Y ; J, \theta_{1}, E\right)$.

Proof. Condition (a) is open by Gromov compactness. Indeed, if (a) were not open at some $K \in \mathcal{J}^{*}\left(X, Y ; J, \theta_{1}, E\right)$ there would exist a sequence $K^{\nu} \rightarrow K$ in $\mathcal{J}\left(X, Y ; J, \theta_{1}\right)$ with nonconstant $K^{\nu}$-holomorphic spheres $f^{\nu}$ of energy at most $E$ contained in $Y$; Gromov compactness would yield a nonconstant $K$-holomorphic sphere of energy at most $E$ contained in $Y$, contradicting $K \in \mathcal{J}^{*}\left(X, Y ; J, \theta_{1}, E\right)$. Openness of condition (b) is proved similarly. Suppose by contradiction that there is a sequence of $K^{\nu} \in \mathcal{J}\left(X, Y ; J, \theta_{1}\right)$ not satisfying (b) and converging to $K \in \mathcal{J}^{*}\left(X, Y ; J, \theta_{1}, E\right)$. Then there exists a sequence of nonconstant $K^{\nu}$-holomorphic spheres $f^{\nu}: S^{\nu} \rightarrow X$ of energy at most $E$ intersecting $Y$ in at most 2 points. Then Gromov compactness yields a nonconstant $K$-holomorphic sphere of energy at most $E$ intersecting $Y$ in at most 2 points, contradicting $K \in \mathcal{J}^{*}\left(X, Y ; J, \theta_{1}, E\right)$.

For density of $\mathcal{J}^{*}\left(X, Y ; J, \theta_{1}, E\right)$, observe that the set of $K \in \mathcal{J}\left(X, Y ; J, \theta_{1}\right)$ satisfying conditions (i) and (ii) of Proposition 8.13 is dense: For condition (i) this is [19, Theorem 3.1.5] (cf. the proof of Lemma 4.1 above). Density of condition (ii) follows from Proposition 6.9 (or Lemma 6.7) with $k=1$, $Z_{1}:=Y$ and $V:=X \backslash Y$. (Here we assume that (i) already holds, and hence by conclusion (a) every nonconstant $K$-holomorphic sphere of energy at most $E$ passes through $V$.)

For the last statement let $J_{0}, J_{1} \in \mathcal{J}^{*}\left(X, Y ; J, \theta_{2}, E\right)$. By Lemma 8.9, they can be connected by a smooth path $\left(J_{\tau}\right)_{\tau \in[0,1]}$ in $\mathcal{J}\left(X, Y ; J, \theta_{1}, E\right)$. By arguments similar to the previous ones, conditions (i) and (ii) of Proposition 8.14 can be achieved for a path $J_{\tau}^{\prime}$ arbitrarily close to $J_{\tau}$. In particular $J^{\prime}(0)$ and $J^{\prime}(1)$ are arbitrarily close to $J_{0}$ and $J_{1}$, respectively. Since $\mathcal{J}^{*}\left(X, Y ; J, \theta_{1}, E\right)$ 
is open, which means that we may assume that $J_{0}$ and $J_{1}$ can be connected in this set.

This concludes the proof.

Extension to two hypersurfaces. The preceding discussion will suffice for the proof of Theorems 1.1 and 1.2. For the proof of Theorem 1.3, we need to extend it to the case of two symplectic hypersurfaces. As most arguments immediately carry over, we will only indicate the required modifications.

As above, consider a closed symplectic manifold $(X, \omega)$ with integral class $[\omega]$. Fix constants

$$
0<\theta_{3}<\theta_{2}<\theta_{1}<\theta_{0}<1
$$

as in Lemmas 8.9 and $8.7(\mathrm{~b})$ and a constant $\varepsilon>0$. For the remainder of this section, we fix a quadruple $\left(J_{0}, Y_{0}, J_{1}, Y_{1}\right)$ consisting of $\omega$-compatible almost complex structures $J_{i}$ and hypersurfaces $Y_{i}$, Poincaré dual to $D_{i}[\omega]$, satisfying

$$
\left\|J_{0}-J_{1}\right\|_{0}<\theta_{3}, \quad \angle_{m}\left(Y_{0}, Y_{1}\right) \geq \varepsilon, \quad \theta\left(Y_{i} ; \omega, J_{i}\right)<\theta_{3} \text { for } i=0,1 .
$$

Here \|\|$_{0}$ denotes the norm with respect to $\omega\left(\cdot, J_{0} \cdot\right)$ and we have set

$$
\angle_{m}\left(Y_{0}, Y_{1}\right):=\inf _{y \in Y_{0} \cap Y_{1}} \angle_{m}\left(T_{y} Y_{0}, T_{y} Y_{1}\right) .
$$

Remark 8.17. The existence of such quadruples follows from the (Stabilization) property in Theorem 8.1. Given a $J_{0}$-holomorphic hypersurface $Y_{0}$, there exists a hypersurface $Y_{1}$ such that the above conditions are satisfied with $J_{0}=J_{1}$. We allow the case $J_{0} \neq J_{1}$ for later use.

By Lemma 8.7(b), there exists an $\omega$-tame almost complex structure $K$ on the vector bundle $\left.T X\right|_{Y_{0} \cap Y_{1}}$ with

$$
\left\|K-J_{0}\right\|_{Y_{0} \cap Y_{1}}<\theta_{2}, \quad K\left(T Y_{0}\right)=T Y_{0}, \quad K\left(T Y_{1}\right)=T Y_{1} .
$$

Arguing as above, using Lemma 8.9 , we can extend $K$ to an $\omega$-tamed almost complex structure on $X$ satisfying

$$
\left\|K-J_{0}\right\|<\theta_{1}, \quad K\left(T Y_{0}\right)=T Y_{0}, \quad J\left(T Y_{1}\right)=T Y_{1} .
$$

Thus the space

$$
\mathcal{J}\left(X, Y_{0} \cup Y_{1}\right):=\left\{K \in \mathcal{J}(X) \mid K\left(T Y_{0}\right)=T Y_{0}, K\left(T Y_{1}\right)=T Y_{1},\right\}
$$

contains a nonempty open subspace

$$
\mathcal{J}\left(X, Y_{0} \cup Y_{1} ; J_{0}, \theta_{1}\right):=\left\{K \in \mathcal{J}\left(X, Y_{0} \cup Y_{1}\right) \mid\left\|K-J_{0}\right\|<\theta_{1}\right\} .
$$

Again, any two elements in $\mathcal{J}\left(X, Y_{0} \cup Y_{1} ; J_{0}, \theta_{1}\right)$ can be connected in the space

$$
\mathcal{J}\left(X, Y_{0} \cup Y_{1} ; J_{0}, \theta_{0}\right):=\left\{K \in \mathcal{J}\left(X, Y_{0} \cup Y_{1}\right) \mid\left\|K-J_{0}\right\|<\theta_{0}\right\} .
$$

We need the following variation of Proposition 8.13. 
Lemma 8.18. Let $\left(J_{0}, Y_{0}, J_{1}, Y_{1}\right)$ be as above, $K \in \mathcal{J}\left(X, Y_{0} \cup Y_{1} ; J_{0}, \theta_{0}\right)$, and $E>0$. Suppose that all moduli spaces of simple $K$-holomorphic spheres in the intersection $Y_{0} \cap Y_{1}$ of energy at most $E$ are smooth manifolds of the expected dimension and $D_{0}+D_{1}>\max \left(D_{*}, D_{*}+n-5\right)$. Then all $K$ holomorphic spheres of energy at most $E$ contained in $Y_{0} \cap Y_{1}$ are constant.

Proof. We have

$$
c_{1}\left(T\left(Y_{0} \cap Y_{1}\right)\right)=c_{1}\left(\left.T X\right|_{Y_{0} \cap Y_{1}}\right)-D_{0}[\omega]-D_{1}[\omega] .
$$

Hence the expected dimension of the moduli space of simple $K$-holomorphic spheres in $Y$ in a homology class $A$ satisfies

$$
\begin{aligned}
\operatorname{ind}(A) & =2(n-2)-6+2\left\langle c_{1}\left(T\left(Y_{0} \cap Y_{1}\right)\right), A\right\rangle \\
& =2 n-10+2\left\langle c_{1}(T X), A\right\rangle-2\left(D_{0}+D_{1}\right) \omega(A) \\
& \leq 2 n-10+2\left(D_{*}-D_{0}-D_{1}\right) \omega(A) .
\end{aligned}
$$

If $A$ is nontrivial, then $\omega(A) \geq 1$ and the hypothesis $D_{0}+D_{1}>\max \left(D_{*}, D_{*}+\right.$ $n-5)$ implies ind $(A)<0$. In view of the regularity hypothesis (and again passing to underlying simple spheres), this shows that all $K$-holomorphic spheres of energy at most $E$ contained in the intersection $Y_{0} \cap Y_{1}$ are constant.

Definition 8.19. For $E>0$ let

$$
\mathcal{J}^{*}\left(X, Y_{0} \cup Y_{1} ; J_{0}, \theta_{1}, E\right) \subset \mathcal{J}\left(X, Y_{0} \cup Y_{1} ; J_{0}, \theta_{1}\right)
$$

be the subset of those $K$ for which the conclusions (a) and (b) of Proposition 8.13 hold for each $Y_{i}$, i.e.,

(a) All $K$-holomorphic spheres of energy at most $E$ contained in $Y_{0} \cup Y_{1}$ are constant.

(b) Every nonconstant $K$-holomorphic sphere of energy at most $E$ in $X$ intersects $Y_{i}$ in at least 3 distinct points in the domain for $i=0,1$.

Note that if $D_{i} \geq D^{*}=2\left(D_{*}+n\right)$ for $i=0,1$, then each $D_{i}$ satisfies the conditions in (a) and (b) of Proposition 8.13 and $D_{0}+D_{1}$ satisfies the hypothesis of Lemma 8.18. We have the following analogue of Corollary 8.16.

Corollary 8.20. Let $\left(J_{0}, Y_{0}, J_{1}, Y_{1}\right)$ be as above and suppose $D_{i} \geq D^{*}$ $(X, \omega, J)$ for $i=0,1$. Then $\mathcal{J}^{*}\left(X, Y_{0} \cup Y_{1} ; J_{0}, \theta_{1}, E\right)$ is open and dense in $\mathcal{J}\left(X, Y_{0} \cup Y_{1} ; J_{0}, \theta_{1}\right)$ for every $E>0$. Moreover, for $\theta_{2}<\theta_{1}$ any two elements in $\mathcal{J}^{*}\left(X, Y_{0} \cup Y_{1} ; J_{0}, \theta_{2}, E\right)$ can be connected by a path in $\mathcal{J}^{*}\left(X, Y_{0} \cup Y_{1}\right.$; $\left.J_{0}, \theta_{1}, E\right)$.

Proof. Openness follows from Gromov compactness as in the proof of Corollary 8.16. For density, it suffices to prove density of the set of $K \in \mathcal{J}\left(X, Y_{0} \cup Y_{1} ; J_{0}, \theta_{1}\right)$ satisfying conditions (i) and (ii) of Proposition 8.13 for both hypersurfaces $Y_{i}$. For this, we first apply [19, Theorem 3.1.5] in 
$Y_{0} \cap Y_{1}$ to conclude density of the set of $K \in \mathcal{J}\left(X, Y_{0} \cup Y_{1} ; J_{0}, \theta_{1}\right)$ for which all moduli spaces of simple $K$-holomorphic spheres in $Y_{0} \cap Y_{1}$ of energy at most

are smooth manifolds of the expected dimension. Then by Lemma 8.18 all $K$-holomorphic spheres contained in $Y_{0} \cap Y_{1}$ are constant. Based on this, we can apply [19, Theorem 3.1.5] in $Y_{0}$ and $Y_{1}$ (varying $K$ on $Y_{0} \cup Y_{1} \backslash\left(Y_{0} \cap Y_{1}\right)$ ) to conclude density of the set of $K \in \mathcal{J}\left(X, Y_{0} \cup Y_{1} ; J_{0}, \theta_{1}\right)$ satisfying condition (i) of Proposition 8.13 for both $Y_{i}$. Finally, we apply Proposition 6.9 (or Lemma 6.7) with $k=1, Z_{1}:=Y_{i}$ and $V:=X \backslash\left(Y_{0} \cup Y_{1}\right)$ (varying $K$ outside $\left.Y_{0} \cup Y_{1}\right)$ to conclude density of condition (ii). This finishes the proof.

Addendum. We conclude this section with a finiteness result for homology classes represented by $K$-holomorphic curves for $\omega$-tamed $K$, although this result will not be needed in this paper. For a compatible almost complex structure $J$ and $0<\theta_{0}<1$ set

$$
\mathcal{J}\left(X ; J, \theta_{0}\right):=\left\{K \in \mathcal{J}(X) \mid\|K-J\|<\theta_{0}\right\} .
$$

Lemma 8.21. For each $E>0$ and $0<\theta_{0}<1$, all homology classes $A \in$ $H_{2}(X ; \mathbb{Z})$ with $\langle\omega, A\rangle \leq E$ that can be represented by a K-holomorphic sphere for some $K \in \mathcal{J}\left(X ; J, \theta_{0}\right)$ are contained in a finite set $\mathcal{A}\left(E, \theta_{0}\right) \subset$ $H_{2}(X ; \mathbb{Z})$ depending only on $\omega, J, E$ and $\theta_{0}$.

Proof. For a 2-form $\sigma$ on $X$ define

$$
\gamma(\sigma):=\inf _{x \in X} \gamma\left(\sigma_{x}\right)
$$

where $\gamma\left(\sigma_{x}\right)$ is defined as above with respect to $(\omega, J)$ and the induced metric on $T X$. Fix a constant $\gamma_{0}$ satisfying

$$
0<\theta_{0}<\gamma_{0}<1
$$

and define

$$
\Omega:=\left\{\sigma \in \Omega^{2}(X) \mid d \sigma=0, \gamma(\sigma)>\gamma_{0}\right\} .
$$

By the properties of $\gamma, \Omega$ is a cone consisting of symplectic forms taming $J$, and $\omega \in \Omega$. Hence its image $[\Omega] \subset H^{2}(X ; \mathbb{R})$ in cohomology is an open cone containing $[\omega]$. Denote by

$$
[\Omega]^{*}:=\left\{A \in H_{2}(X ; \mathbb{R}) \mid\langle\sigma, A\rangle \geq 0 \text { for all } \sigma \in \Omega\right\}
$$

the dual cone in homology. Then for given $E>0$ the set $\left\{A \in[\Omega]^{*} \mid\langle\omega, A\rangle \leq\right.$ $E\}$ is bounded. (This is a general property of cones in finite dimensions which can be seen as follows. Write $A=\sum_{j} A_{j} v_{j}$ in a basis of cohomology whose dual basis satisfies $[\omega] \pm v_{j}^{*} \in[\Omega]$ for all $j$; then $0 \leq\left\langle\omega \pm v_{j}^{*}, A\right\rangle \leq E \pm A_{j}$ implies $\left|A_{j}\right| \leq E$ for all $j$.) Therefore, the set

$$
\mathcal{A}:=\left\{A \in[\Omega]^{*} \mid\langle\omega, A\rangle \leq E\right\} \cap H_{2}(X ; \mathbb{Z})
$$

is finite. Since $\theta_{0}<\gamma_{0}$, Lemma $8.5(\mathrm{~b})$ and the definition of $\Omega$ show that $\mathcal{J}\left(X ; J, \theta_{0}\right)$ consists of almost complex structures which are tamed 
by all $\sigma \in \Omega$. Now suppose that a class $A \in H_{2}(X ; \mathbb{Z})$ with $\langle\omega, A\rangle \leq E$ can be represented by a $K$-holomorphic sphere $f: S^{2} \rightarrow X$ for some $K \in \mathcal{J}\left(X ; J, \theta_{0}\right)$. Then $\langle\sigma, A\rangle=\int_{S^{2}} f^{*} \sigma \geq 0$ for all $\sigma \in \Omega$, which implies $A \in \mathcal{A}$.

\section{Proof of transversality}

In this section we prove Theorems 1.1 and 1.2 from the introduction, as well as their extensions to the case of two hypersurfaces. Throughout this section, $(X, \omega)$ denotes a closed symplectic manifold of dimension $2 n$.

Before beginning the proofs, let us summarize the transversality results proved in Sections 5 and 6 in the form we will use them. Here conditions (a) follow from Corollaries 5.8 and 5.9, and conditions (b) follow from Corollaries 6.11 and 6.12 .

Proposition 9.1. Let $\bar{J} \in \mathcal{J}$. Let $\mathcal{Z}$ be a countable collection of $\bar{J}$-complex manifolds not equal to $X$ and set $V:=X \backslash \cup_{Z \in \mathcal{Z}} Z$, thus $\mathcal{J}_{\ell+1}(V)$ is the set of coherent almost complex structures that agree with $\bar{J}$ along $\cup_{Z \in \mathcal{Z} Z}$. Then for all $\ell \geq 3$ there exist Baire sets $\mathcal{J}_{\ell+1}^{\text {reg }}(V ; \mathcal{Z} ; \bar{J}) \subset \mathcal{J}_{\ell+1}(V)$ such that for each $I$-stable $k$-labelled tree $T$, homology classes $A_{\alpha} \in H_{2}(X ; \mathbb{Z})$ for $\alpha \in T$, $Z_{i} \in \mathcal{Z} \cup\{X\}$ and integers $\ell_{i} \geq-1$ for $i \in R$ (the reduced index set), and $K \in \mathcal{J}_{|I|+1}^{\text {reg }}(V ; \mathcal{Z} ; \bar{J})$ the following conditions are satisfied.

(a) The moduli space $\mathcal{M}_{T}^{*}\left(\left\{A_{\alpha}\right\}, K ;\left\{Z_{i}\right\}\right)$ of stable $K$-holomorphic maps modelled over $\left(T,\left\{A_{\alpha}\right\}\right)$, passing through $Z_{i}$ at the points $z_{i}$ for $i \in R$ and without a nonconstant component entirely contained in a $Z \in \mathcal{Z}$, is a smooth manifold of dimension

$$
\begin{aligned}
\operatorname{dim} \mathcal{M}_{T}^{*}\left(\left\{A_{\alpha}\right\}, K ;\left\{Z_{i}\right\}\right)= & 2 n-6+\sum_{\alpha \in T} 2 c_{1}\left(A_{\alpha}\right)+2 k-2 e(T) \\
& -2 \sum_{i \in R} \operatorname{codim}_{\mathbb{C}} Z_{i} .
\end{aligned}
$$

Moreover, the evaluation map $\mathrm{ev}^{k}$ factors as

$$
\mathrm{ev}^{k}: \mathcal{M}_{T}^{*}\left(\left\{A_{\alpha}\right\}, K ;\left\{Z_{i}\right\}\right) \longrightarrow \mathcal{M}_{\pi_{R}(T)}^{*}\left(\left\{A_{\alpha}\right\}, K ;\left\{Z_{i}\right\}\right) \longrightarrow X^{k}
$$

through a smooth manifold of dimension

$$
\begin{aligned}
\operatorname{dim} \mathcal{M}_{\pi_{R}(T)}^{*}\left(\left\{A_{\alpha}\right\}, K ;\left\{Z_{i}\right\}\right)= & 2 n-6+\sum_{\alpha \in T} 2 c_{1}\left(A_{\alpha}\right)+2|R| \\
& -2 e\left(\pi_{R}(T)\right)-2 \sum_{i \in R} \operatorname{codim}_{\mathbb{C}} Z_{i} .
\end{aligned}
$$

(b) Suppose that $A_{\alpha} \neq 0$ for a unique vertex $\alpha$ (so the reduced index set $R$ labels the special points on $\alpha)$. Then the moduli space $\mathcal{M}_{T}^{*}\left(A_{\alpha}, K\right.$; $\left.\left\{Z_{i}, \ell_{i}\right\}\right)$ of stable $K$-holomorphic maps modelled over $\left(T, A_{\alpha}\right)$, tangent 
to $Z_{i}$ of order $\ell_{i}$ at the special points $z_{\alpha i}$ on the component $\alpha$ and not entirely contained in a $Z \in \mathcal{Z}$, is a smooth manifold of dimension

$$
\begin{aligned}
\operatorname{dim} \mathcal{M}_{T}^{*}\left(A_{\alpha}, K ;\left\{Z_{i}, \ell_{i}\right\}\right)= & 2 n-6+2 c_{1}\left(A_{\alpha}\right)+2 k-2 e(T) \\
& -2 \sum_{i \in R}\left(\ell_{i}+1\right) \operatorname{codim}_{\mathbb{C}} Z_{i} .
\end{aligned}
$$

Moreover, the evaluation map $\mathrm{ev}^{k}$ factors as

$$
\mathrm{ev}^{k}: \mathcal{M}_{T}^{*}\left(A_{\alpha}, K ;\left\{Z_{i}, \ell_{i}\right\}\right) \longrightarrow \mathcal{M}_{|R|}^{*}\left(A_{\alpha}, K ;\left\{Z_{i}, \ell_{i}\right\}\right) \longrightarrow X^{k}
$$

through a smooth manifold of dimension

$$
\begin{aligned}
\operatorname{dim} \mathcal{M}_{|R|}^{*}\left(A_{\alpha}, K ;\left\{Z_{i}, \ell_{i}\right\}\right)= & 2 n-6+2 c_{1}\left(A_{\alpha}\right)+2|R| \\
& -2 \sum_{i \in R}\left(\ell_{i}+1\right) \operatorname{codim}_{\mathbb{C}} Z_{i} .
\end{aligned}
$$

We first turn to the proof of Theorem 1.1. From now on we suppose that $[\omega] \in H^{2}(X ; \mathbb{Z})$, and we have fixed constants $\varepsilon>0$ and $0<\theta_{3}<\theta_{2}<\theta_{1}<$ $\theta_{0}<1$ as in the previous section. Also recall the definition

$$
D^{*}(X, \omega, J)=2 D_{*}(X, \omega, J)+2 n,
$$

where $D_{*}(X, \omega, J)$ is the constant from Lemma 8.11.

Definition 9.2. A Donaldson pair of degree $D$ is a pair $(J, Y)$ consisting of an $\omega$-compatible almost complex structure $J$ and a hypersurface $Y^{2 n-2} \subset X$ Poincaré dual to $D[\omega]$ satisfying

$$
\theta(Y ; \omega, J)<\theta_{3}, \quad D \geq D^{*}(X, \omega, J) .
$$

Remark 9.3. The proof of Theorems 1.1 and 1.2 only uses the weaker hypothesis $\theta(Y ; \omega, J)<\theta_{2}$. The stronger hypothesis is needed in the proof of Theorem 1.3.

Let $(J, Y)$ be a Donaldson pair of degree $D$ on $(X, \omega)$. To each integer multiple $\ell \geq 3$ of $D$, we associate the energy

$$
E_{\ell}:=\ell / D
$$

For this energy, let $\mathcal{J}^{*}\left(X, Y ; J, \theta_{1}, E_{\ell}\right) \subset \mathcal{J}\left(X, Y ; J, \theta_{1}\right)$ be the set of almost complex structures from Definition 8.15, which is open and dense by Corollary 8.16. Let $\mathcal{J}_{\ell+1}$ be the set of coherent almost complex structures from Section 3.

Definition 9.4. We call an open subset $B^{*} \subset \mathcal{J}^{*}\left(X, Y ; J, \theta_{1}, E_{\ell}\right) \theta_{2}$-contractible if it is contractible in $\mathcal{J}^{*}\left(X, Y ; J, \theta_{1}, E_{\ell}\right)$ to a point of $\mathcal{J}^{*}(X, Y ; J$, 
$\left.\theta_{2}, E_{\ell}\right)$. Then we define

$$
\begin{aligned}
& \mathcal{J}_{\ell+1}\left(X, Y ; J, \theta_{1}\right) \\
& :=\left\{K \in \mathcal{J}_{\ell+1} \mid K(\zeta) \in \mathcal{J}\left(X, Y ; J, \theta_{1}\right) \text { for all } \zeta \in \overline{\mathcal{M}}_{\ell+1},\right. \\
& \left.\left.K(\zeta)\right|_{Y} \text { independent of } \zeta\right\} \\
& \mathcal{J}_{\ell+1}^{*}\left(X, Y ; J, \theta_{1}\right) \\
& :=\left\{K \in \mathcal{J}_{\ell+1} \mid K(\zeta) \in B^{*} \text { for all } \zeta \in \overline{\mathcal{M}}_{\ell+1},\right. \\
& \left.B^{*} \subset \mathcal{J}^{*}\left(X, Y ; J, \theta_{1}, E_{\ell}\right) \theta_{2} \text {-contractible, }\left.K(\zeta)\right|_{Y} \text { independent of } \zeta\right\} \text {. }
\end{aligned}
$$

Note that $\mathcal{J}_{\ell+1}\left(X, Y ; J, \theta_{1}\right)$ is a Banach manifold, and $\mathcal{J}_{\ell+1}^{*}\left(X, Y ; J, \theta_{1}\right) \subset$ $\mathcal{J}_{\ell+1}\left(X, Y ; J, \theta_{1}\right)$ is open and nonempty by Corollary 8.16 and Lemma 8.9.

Proof of Theorem 1.1. Fix a Donaldson pair $(J, Y)$ of degree $D$. Consider a homology class $A \in H_{2}(X ; \mathbb{Z})$ with $\omega(A)>0$ and let

$$
\ell:=D \omega(A)=[Y] \cdot A,
$$

thus $E_{\ell}=\omega(A)$. For $K \in \mathcal{J}_{\ell+1}^{*}\left(X, Y ; J, \theta_{1}\right)$ and $k \geq 0$ define the moduli space

$$
\begin{gathered}
\mathcal{M}_{k+\ell}(A, K ; Y):=\left\{(\mathbf{z}, f) \mid z_{i} \in S^{2} \text { distinct, } f: S^{2} \longrightarrow X, \bar{\partial}_{K} f=0,[f]=A,\right. \\
\left.f\left(z_{i}\right) \in Y \text { for } i=k+1, \ldots, k+\ell\right\} / \operatorname{Aut}\left(S^{2}\right)
\end{gathered}
$$

of $K$-holomorphic spheres in the class $A$ passing at the last $\ell$ points through $Y$. More generally, for an $\ell$-stable (i.e., stable after removing the first $k$ marked points) $(k+\ell)$-labelled tree $T$, homology classes $A_{\alpha} \in H_{2}(X ; \mathbb{Z})$ for $\alpha \in T$ with $\sum A_{\alpha}=A$, and $K \in \mathcal{J}_{\ell+1}^{*}\left(X, Y ; J, \theta_{1}\right)$ consider the moduli space

$$
\begin{aligned}
& \mathcal{M}_{T}\left(\left\{A_{\alpha}\right\}, K ; Y\right) \\
& \quad:=\left\{(\mathbf{z}, \mathbf{f}) \in \mathcal{M}_{T}\left(\left\{A_{\alpha}\right\}, K\right) \mid f_{\alpha_{i}}\left(z_{i}\right) \in Y \text { for } i=k+1, \ldots, k+\ell\right\}
\end{aligned}
$$

of stable maps modelled over $\left(T,\left\{A_{\alpha}\right\}\right)$ passing at the last $\ell$ marked points through $Y$. Note that for elements of $\mathcal{M}_{T}\left(\left\{A_{\alpha}\right\}, K ; Y\right)$ every nonconstant component passes through $V:=X \backslash Y$. This follows from property (a) in Definition 8.15 because $\left.K(\zeta)\right|_{Y}$ is independent of $\zeta$ and belongs to $\mathcal{J}^{*}\left(X, Y ; J, \theta_{1}, E_{\ell}\right)$.

For any $\bar{J} \in \mathcal{J}^{*}\left(X, Y ; J, \theta_{1}, E_{\ell}\right)$, we apply Proposition 9.1 with $k$ replaced by $k+\ell$, the index set $I=\{k+1, \ldots, k+\ell\}$ and the collection $\mathcal{Z}=\{Y\}$, thus $V=X \backslash Y$. Let $\mathcal{J}_{\ell+1}^{\text {reg }}(V ;\{Y\} ; \bar{J}) \subset \mathcal{J}_{\ell+1}(V)$ be the Baire set provided by Proposition 9.1 and set

$\mathcal{J}_{\ell+1}^{\mathrm{reg}}\left(X, Y ; J, \theta_{1}\right):=\bigcup_{\bar{J} \in \mathcal{J}^{*}\left(X, Y ; J, \theta_{1}, E_{\ell}\right)} \mathcal{J}_{\ell+1}^{\mathrm{reg}}(V ;\{Y\} ; \bar{J}) \cap \mathcal{J}_{\ell+1}^{*}\left(X, Y ; J, \theta_{1}, E_{\ell}\right)$.

Note that elements in $\mathcal{J}_{\ell+1}^{\text {reg }}(V ;\{Y\} ; \bar{J})$ not necessarily satisfy the conditions (a) and (b) of Definition 8.19 nor are they close to $\bar{J}$. Hence we intersect 
here with $\mathcal{J}_{\ell+1}^{*}\left(X, Y ; J, \theta_{1}, E_{\ell}\right)$. Then for $K \in \mathcal{J}_{\ell+1}^{\text {reg }}\left(X, Y ; J, \theta_{1}\right)$ the following holds. Let $A \in H_{2}(X ; \mathbb{Z})$ with $\ell=D \omega(A)$, and $T$ be an $\ell$-stable $(k+\ell)$-labelled tree with weights $A_{\alpha}, \sum A_{\alpha}=A$, such that every ghost tree contains at most one of the last $\ell$ marked points. This condition implies that the reduced index set $R$ satisfies $\{k+1, \ldots, k+\ell\} \subset R$. So by Proposition 9.1(a), the moduli space $\mathcal{M}_{T}\left(\left\{A_{\alpha}\right\}, K ; Y\right)$ of stable $K$-holomorphic maps modelled over $\left(T,\left\{A_{\alpha}\right\}\right)$ and passing through $Y$ at the last $\ell$ marked points is a smooth manifold of dimension

$$
\begin{aligned}
\operatorname{dim}_{\mathbb{R}} \mathcal{M}_{T}\left(\left\{A_{\alpha}\right\}, K ; Y\right) & =2 n-6+2 c_{1}(A)+2(k+\ell)-2 e(T)-2 \ell \operatorname{codim}_{\mathbb{C}}(Y) \\
& =2 n-6+2 c_{1}(A)+2 k-2 e(T) \\
& =2 d-2 e(T) .
\end{aligned}
$$

This proves Theorem 1.1.

In particular, applying Theorem 1.1 to the tree $T$ with one vertex (which has no ghost components), it follows that the moduli space $\mathcal{M}_{k+\ell}(A, K ; Y)$ is a smooth manifold of dimension

$$
\operatorname{dim}_{\mathbb{R}} \mathcal{M}_{k+\ell}(A, K ; Y)=2 n-6+2 k+2 c_{1}(A)=2 d .
$$

Next we turn to the proof of Theorem 1.2. We need to show that the evaluation map at the first $k$ points

$$
\mathrm{ev}^{k}: \mathcal{M}_{k+\ell}(A, K ; Y) \longmapsto X^{k}
$$

is a $2 d$-dimensional pseudocycle. The following result is the key to the proof.

Proposition 9.5. Let $K \in \mathcal{J}_{\ell+1}^{*}\left(X, Y ; J, \theta_{1}\right), k \geq 0$, and $A \in H_{2}(X ; \mathbb{Z})$ with $\ell=D \omega(A)$. Suppose that a sequence $\left[f^{\nu}\right] \in \mathcal{M}_{k+\ell}(A, K ; Y)$ converges in the sense of Gromov to a stable map $[\mathbf{z}, \mathbf{f}]$. Then the nodal curve $\mathbf{z}$ is $\ell$-stable.

Proof. Arguing by contradiction, suppose that $\mathbf{z}$ is not $\ell$-stable. Then there exists a nonconstant component $f_{\alpha}$ of $\mathbf{f}$ whose domain $S_{\alpha}$ carries at most two special points after removing the first $k$ marked points. According to condition (3) after Theorem 5.2, $\mathbf{f}$ is $K_{\pi_{\ell}(\mathbf{z})}$-holomorphic, where $\pi_{\ell}$ is defined by removing the first $k$ marked points and then stabilizing. As $\pi_{\ell}$ maps $S_{\alpha}$ to a point, this means that $\bar{\partial}_{K_{\alpha}} f_{\alpha}=0$ for some $K_{\alpha} \in \mathcal{J}^{*}\left(X, Y ; J, \theta_{1}\right)$ (not depending on points on $S_{\alpha}$ ).

By condition (a) in Definition 8.15 (since $f_{\alpha}$ is nonconstant), $f_{\alpha}$ is not contained in $Y$, and by condition (b) it intersects $Y$ in at least three points in the domain. Thus $f_{\alpha}$ must intersect $Y$ at a point that is neither a node nor one of the last $\ell$ marked points. By Proposition 7.1, intersections persist under small perturbations. So the definition of Gromov convergence implies that for $\nu$ sufficiently large, $\mathbf{f}^{\nu}$ intersects $Y$ at a point which is not one of the last $\ell$ marked points. On the other hand, by Proposition 7.1 each of the last $\ell$ marked points contributes at least one to the intersection number (since 
$f_{\nu}: S^{2} \rightarrow X$ is a smooth $K$-holomorphic curve with distinct marked points). Hence we conclude $[Y] \cdot\left[\mathbf{f}^{\nu}\right]>\ell=[Y] \cdot A$, in contradiction to $\left[\mathbf{f}^{\nu}\right]=A$.

Proposition 9.6. For all $K \in \mathcal{J}_{\ell+1}^{\mathrm{reg}}\left(X, Y ; J, \theta_{1}\right)$ (as defined above) the following holds. Let $A \in H_{2}(X ; \mathbb{Z})$ with $\ell=D \omega(A), T$ be an $\ell$-stable $(k+\ell)$ labelled tree with more than one vertex, and $A_{\alpha} \in H_{2}(X ; \mathbb{Z})$ for $\alpha \in T$ with $\sum A_{\alpha}=A$. Then the evaluation map ev ${ }^{k}: \mathcal{M}_{T}\left(\left\{A_{\alpha}\right\}, K ; Y\right) \rightarrow X^{k}$ factors through a manifold of dimension at most $2 d-2$.

Proof. If every ghost tree in $T$ contains at most one of the last $\ell$ marked points the conclusion of the proposition follows from Theorem 1.1 because $e(T) \geq 1$ if $T$ has more than one vertex.

It remains to analyze the case that there exists a ghost tree $T^{\prime} \subset T$ containing at least two of the last $\ell$ marked points. Let $R$ be the reduced index set (retaining only the point with maximal index $i$ on each ghost tree) and $\bar{T}:=\pi_{R}(T)$ the tree obtained by removing the marked points outside $R$ and stabilizing. By Proposition 9.1(a), the evaluation map ev ${ }^{k}$ factors through the manifold $\mathcal{M}_{\bar{T}}^{*}\left(\left\{A_{\alpha}\right\}, K ;\left\{Z_{i}\right\}\right)$, where $Z_{i}=X$ for $i=1, \ldots, k$ and $Z_{i}=Y$ for $i=k+1, \ldots, k+\ell$. Now we distinguish two cases.

Case 1. $\bar{T}$ has more than one vertex. Then by Proposition 9.1(a), the evaluation map ev ${ }^{k}$ factors through the moduli space $\mathcal{M}_{\bar{T}}^{*}\left(\left\{A_{\alpha}\right\}, K ;\left\{Z_{i}\right\}\right)$ of dimension at most $2 d-e(\bar{T}) \leq 2 d-2$.

Case 2. $\bar{T}$ has only one vertex. This happens iff $A_{\alpha} \neq 0$ for exactly one vertex $\alpha$, so we are in the situation of Proposition 9.1(b). Now recall that by assumption $T$ had at least two of the last $\ell$ marked points on the same ghost tree $T^{\prime}$. So Lemma 7.2 shows that for each $(\mathbf{z}, \mathbf{f}) \in \mathcal{M}_{T}\left(\left\{A_{\alpha}\right\}, K ; Y\right)$ the map $f_{\alpha}$ has local intersection number with $Y$ at least 2 (hence order of tangency at least 1) at the marked point $z_{\alpha j}, j \geq k+1$, at which the ghost tree $T^{\prime}$ is attached. Hence by Proposition 9.1(b), the evaluation map ev ${ }^{k}$ factors through the moduli space $\mathcal{M}_{|R|}^{*}\left(A_{\alpha}, K ;\left\{Z_{i}, \ell_{i}\right\}\right)$, where $Z_{i}=Y$ and $\ell_{i} \geq 0$ for all $i \geq k+1$, and $\ell_{j} \geq 1$. So the dimension formula in Proposition 9.1 (b) shows that

$$
\operatorname{dim} \mathcal{M}_{|R|}^{*}\left(A_{\alpha}, K ;\left\{Z_{i}, \ell_{i}\right\}\right) \leq 2 d-2 \sum_{i \in R, i \geq k+1} \ell_{i} \operatorname{codim}_{\mathbb{C}} Y \leq 2 d-2 .
$$

This proves Proposition 9.6.

Proof of Theorem 1.2. Theorem 1.2 follows immediately from Propositions 9.5, 9.6, and the definition of a pseudo-cycle.

Extension to two hypersurfaces. Now we extend the preceding discussion to the case of two hypersurfaces. 
Definition 9.7. A Donaldson quadruple of bi-degree $\left(D_{0}, D_{1}\right)$ is a quadruple $\left(J_{0}, Y_{0}, J_{1}, Y_{1}\right)$ consisting of two Donaldson pairs $\left(J_{i}, Y_{i}\right)$ of degrees $D_{i}$ satisfying

$$
\angle_{m}\left(Y_{0}, Y_{1}\right) \geq \varepsilon, \quad\left\|J_{0}-J_{1}\right\|_{0}<\theta_{3} .
$$

The following is the analogue of Theorem 1.1 in this case.

Theorem 9.8. Let $\left(J_{0}, Y_{0}, J_{1}, Y_{1}\right)$ be a Donaldson quadruple on $(X, \omega)$ of bi-degree $\left(D_{0}, D_{1}\right)$. Then for all integers $\bar{\ell} \geq 3$ there exist nonempty sets $\mathcal{J}_{\bar{\ell}+1}^{\text {reg }}\left(X, Y_{0} \cup Y_{1} ; J_{0}, \theta_{1}\right) \subset \mathcal{J}_{\bar{\ell}+1}\left(X, Y_{0} \cup Y_{1} ; J_{0}, \theta_{1}\right)$ with the following property. Let $A \in H_{2}(X ; \mathbb{Z})$ be a homology class with $\omega(A)>0$ and set $\ell_{i}:=D_{i} \omega(A)$ and $\ell:=\ell_{0}+\ell_{1}$. Let $T$ be an I-stable $(k+\ell)$-labelled tree for some $k \geq 0$ and a subset $I \subset\{k+1, \ldots, k+\ell\}$ satisfying $|I| \geq 3$ and $|I| \geq \min \left(D_{0}, D_{1}\right) \omega(A)$. Let $A_{\alpha} \in H_{2}(X ; \mathbb{Z})$ for $\alpha \in T$ such that $\sum A_{\alpha}=A$ and such that every ghost tree in $\left(T,\left\{A_{\alpha}\right\}\right)$ contains at most one of the last $\ell$ marked points. Then for each $K \in \mathcal{J}_{|I|+1}^{\mathrm{reg}}\left(X, Y_{0} \cup Y_{1} ; J_{0}, \theta_{1}\right)$ the moduli space $\mathcal{M}_{T}\left(\left\{A_{\alpha}\right\}, K ; Y_{0} \cup Y_{1}\right)$ of stable $K$-holomorphic spheres modelled over $\left(T\left\{A_{\alpha}\right\}\right)$, mapping the middle $\ell_{0}$ marked points to $Y_{0}$ and the last $\ell_{1}$ marked points to $Y_{1}$, is a smooth manifold of real dimension

$$
\operatorname{dim}_{\mathbb{R}} \mathcal{M}_{T}\left(\left\{A_{\alpha}\right\}, K ; Y_{0} \cup Y_{1}\right)=2\left(n-3+k+c_{1}(A)-e(T)\right) .
$$

Proof. To each integer $\bar{\ell} \geq 3$ we associate the energy

$$
E_{\bar{\ell}}:=\bar{\ell} / \min \left(D_{0}, D_{1}\right) \text {. }
$$

(We use $\bar{\ell}$ because $\ell$ will have a different meaning below.) For this energy, let $\mathcal{J}^{*}\left(X, Y_{0} \cup Y_{1} ; J_{0}, \theta_{1}, E_{\bar{\ell}}\right) \subset \mathcal{J}\left(X, Y_{0} \cup Y_{1} ; J_{0}, \theta_{1}\right)$ be the set of almost complex structures from Definition 8.19, which is open and dense by Corollary 8.20. Let $\mathcal{J}_{\bar{\ell}+1}$ be the set of coherent almost complex structures from Section 3 .

Definition 9.9. We call an open subset $B^{*} \subset \mathcal{J}^{*}\left(X, Y_{0} \cup Y_{1} ; J_{0}, \theta_{1}, E_{\bar{\ell}}\right) \theta_{2}$ contractible if it is contractible in $\mathcal{J}^{*}\left(X, Y_{0} \cup Y_{1} ; J_{0}, \theta_{1}, E_{\bar{\ell}}\right)$ to a point in $\mathcal{J}^{*}\left(X, Y_{0} \cup Y_{1} ; J_{0}, \theta_{2}, E_{\bar{\ell}}\right)$. Then we define

$$
\begin{aligned}
\mathcal{J}_{\bar{\ell}+1}\left(X, Y_{0} \cup Y_{1} ; J_{0}, \theta_{1}\right) & =\left\{K \in \mathcal{J}_{\bar{\ell}+1} \mid K(\zeta) \in \mathcal{J}\left(X, Y_{0} \cup Y_{1} ; J_{0}, \theta_{1}\right) \text { for all } \zeta \in \overline{\mathcal{M}}_{\bar{\ell}+1},\right. \\
& \left.\left.K(\zeta)\right|_{Y_{0} \cup Y_{1}} \quad \text { independent of } \zeta\right\} \\
\mathcal{J}_{\bar{\ell}+1}^{*}\left(X, Y_{0} \cup Y_{1} ; J_{0}, \theta_{1}\right) & \\
:= & \left\{K \in \mathcal{J}_{\bar{\ell}+1} \mid K(\zeta) \in B^{*} \text { for all } \zeta \in \overline{\mathcal{M}}_{\bar{\ell}+1},\right. \\
& B^{*} \subset \mathcal{J}^{*}\left(X, Y_{0} \cup Y_{1} ; J_{0}, \theta_{1}, E_{\bar{\ell}}\right) \theta_{2} \text {-contractible, } \\
& \left.\left.K(\zeta)\right|_{Y_{0} \cup Y_{1}} \quad \text { independent of } \zeta\right\} .
\end{aligned}
$$


Note that $\mathcal{J}_{\bar{\ell}+1}\left(X, Y_{0} \cup Y_{1} ; J_{0}, \theta_{1}\right)$ is a Banach manifold, and $\mathcal{J}_{\bar{\ell}+1}^{*}\left(X, Y_{0} \cup\right.$ $\left.Y_{1} ; J_{0}, \theta_{1}\right) \subset \mathcal{J}_{\bar{\ell}+1}\left(X, Y_{0} \cup Y_{1} ; J_{0}, \theta_{1}\right)$ is open and nonempty by Corollary 8.20 and Lemma 8.9.

Now consider a homology class $A \in H_{2}(X ; \mathbb{Z})$ with $\omega(A)>0$ and set

$$
\ell_{i}:=D_{i} \omega(A)=\left[Y_{i}\right] \cdot A, \quad \ell:=\ell_{0}+\ell_{1} .
$$

For $k \geq 0$ we denote points in the Deligne-Mumford space $\overline{\mathcal{M}}_{k+\ell_{0}+\ell_{1}+1}$ by

$$
\bar{z}=\left(z_{0}, \ldots, z_{k}, z_{k+1}, \ldots, z_{k+\ell_{0}}, z_{k+\ell_{0}+1}, \ldots, z_{k+\ell_{0}+\ell_{1}}\right),
$$

so the first components after $z_{0}$ correspond to $k$, the middle ones to $\ell_{0}$, and the last ones to $\ell_{1}$. Fix a subset $I \subset\{k+1, \ldots, k+\ell\}$ with $|I| \geq 3$ and $|I| \geq \min \left(D_{0}, D_{1}\right) \omega(A)$, hence

$$
\omega(A) \leq E_{|I|}
$$

Denote by

$$
\pi_{I}: \overline{\mathcal{M}}_{k+\ell_{0}+\ell_{1}+1} \longrightarrow \overline{\mathcal{M}}_{|I|+1}
$$

the obvious projection (forgetting the marked points outside $I$ and stabilizing). Recall that a $k+\ell_{0}+\ell_{1}$-labelled tree is called $I$-stable if it is still stable after removing the marked points outside $I$.

For $K \in \mathcal{J}_{|I|+1}^{*}\left(X, Y_{0} \cup Y_{1} ; J_{0}, \theta_{1}\right)$ (as defined above with $\left.\bar{\ell}=|I|\right)$ define the moduli space

$$
\begin{aligned}
\mathcal{M}_{k+\ell_{0}+\ell_{1}}\left(A, K ; Y_{0} \cup Y_{1}\right):=\left\{(\mathbf{z}, f) \mid z_{i} \in S^{2} \text { distinct, } f: S^{2} \longrightarrow X,\right. \\
\bar{\partial}_{\pi_{I}^{*} K} f=0,[f]=A, f\left(z_{i}\right) \in Y_{0} \text { for } k+1 \leq i \leq k+\ell_{0}, \\
\left.f\left(z_{i}\right) \in Y_{1} \text { for } k+\ell_{0}+1 \leq i \leq k+\ell_{0}+\ell_{1}\right\} / \operatorname{Aut}\left(S^{2}\right)
\end{aligned}
$$

of $K$-holomorphic spheres in the class $A$ passing at the middle $\ell_{0}$ points through $Y_{0}$ and at the last $\ell_{1}$ points through $Y_{1}$. More generally, for an $I$-stable $\left(k+\ell_{0}+\ell_{1}\right)$-labelled tree $T$, homology classes $A_{\alpha} \in H_{2}(X ; \mathbb{Z})$ with $\sum A_{\alpha}=A$, and $K \in \mathcal{J}_{|I|+1}^{*}\left(X, Y_{0} \cup Y_{1} ; J_{0}, \theta_{1}\right)$ consider the moduli space

$$
\begin{aligned}
& \mathcal{M}_{T}\left(\left\{A_{\alpha}\right\}, K ; Y_{0} \cup Y_{1}\right):=\left\{(\mathbf{z}, \mathbf{f}) \in \mathcal{M}_{T}\left(\left\{A_{\alpha}\right\}, K\right) \mid f_{\alpha_{i}}\left(z_{i}\right) \in Y_{0}\right. \\
& \left.\quad \text { for } k+1 \leq i \leq k+\ell_{0}, f_{\alpha_{i}}\left(z_{i}\right) \in Y_{1} \text { for } k+\ell_{0}+1 \leq i \leq k+\ell_{0}+\ell_{1}\right\}
\end{aligned}
$$

of stable maps modelled over $\left(T,\left\{A_{\alpha}\right\}\right)$, passing at the middle $\ell_{0}$ marked points through $Y_{0}$ and at the last $\ell_{1}$ points through $Y_{1}$. Note that for elements of $\mathcal{M}_{T}\left(\left\{A_{\alpha}\right\}, K ; Y_{0} \cup Y_{1}\right)$ every nonconstant component passes through $V:=X \backslash\left(Y_{0} \cup Y_{1}\right)$ : This follows from property (a) in Definition 8.19 because $\left.K(\zeta)\right|_{Y_{0} \cup Y_{1}}$ is independent of $\zeta$ and belongs to $\mathcal{J}^{*}\left(X, Y_{0} \cup Y_{1} ; J_{0}, \theta_{1}, E_{|I|}\right)$, and $\omega(A) \leq E_{|I|}$. 
For any $\bar{J}_{0} \in \mathcal{J}^{*}\left(X, Y_{0} \cup Y_{1} ; J_{0}, \theta_{1}, E_{|I|}\right)$ we apply Proposition 9.1 with $k$ replaced by $k+\ell$, the index set $I \subset\{k+1, \ldots, k+\ell\}$ above and the collection

$$
\mathcal{Z}=\left\{Y_{0}, Y_{1}, Y_{0} \cap Y_{1}\right\},
$$

thus $V=X \backslash\left(Y_{0} \cup Y_{1}\right)$. Let $\mathcal{J}_{|I|+1}^{\text {reg }}\left(V ;\left\{Y_{0}, Y_{1}, Y_{0} \cap Y_{1}\right\} ; \bar{J}_{0}\right) \subset \mathcal{J}_{|I|+1}(V)$ be the Baire set provided by Proposition 9.1 and set

$$
\begin{aligned}
& \mathcal{J}_{|I|+1}^{\mathrm{reg}}\left(X, Y_{0} \cup Y_{1} ; J_{0}, \theta_{1}\right):= \\
& \qquad \underset{\bar{J}_{0} \in \mathcal{J}^{*}\left(X, Y_{0} \cup Y_{1} ; J_{0}, \theta_{1}, E_{|I|}\right)}{\mathcal{J}_{|I|+1}^{\mathrm{reg}}\left(V ;\left\{Y_{0}, Y_{1}, Y_{0} \cap Y_{1}\right\} ; \bar{J}_{0}\right) \cap \mathcal{J}_{|I|+1}^{*}\left(X, Y_{0} \cup Y_{1} ; J_{0}, \theta_{1}, E_{|I|}\right) .}
\end{aligned}
$$

Then for $K \in \mathcal{J}_{|I|+1}^{\text {reg }}\left(X, Y_{0} \cup Y_{1} ; J_{0}, \theta_{1}\right)$ the following holds. Let $A \in H_{2}$ $(X ; \mathbb{Z})$ with $\ell_{i}=D_{i} \omega(A)$ and $\ell=\ell_{0}+\ell_{1}$. Let $T$ be an $I$-stable (with $I$ as above) $(k+\ell)$-labelled tree with weights $A_{\alpha}, \sum A_{\alpha}=A$, such that every ghost tree contains at most one of the last $\ell$ marked points. This condition implies that the reduced index set $R$ satisfies $\{k+1, \ldots, k+\ell\} \subset R$. So by Proposition 9.1 (a), the moduli space $\mathcal{M}_{T}\left(\left\{A_{\alpha}\right\}, K ; Y_{0} \cup Y_{1}\right)$ is a smooth manifold of dimension

$$
\begin{aligned}
\operatorname{dim}_{\mathbb{R}} \mathcal{M}_{T}\left(\left\{A_{\alpha}\right\}, K ; Y_{0} \cup Y_{1}\right)= & 2 n-6+2 c_{1}(A)+2(k+\ell)-2 e(T) \\
& -2 \ell_{0} \operatorname{codim}_{\mathbb{C}}\left(Y_{0}\right)-2 \ell_{1} \operatorname{codim}_{\mathbb{C}}\left(Y_{1}\right) \\
= & 2 n-6+2 c_{1}(A)+2 k-2 e(T) \\
= & 2 d-2 e(T) .
\end{aligned}
$$

This proves Theorem 9.8.

In particular, applying Theorem 9.8 to the tree $T$ with one vertex (which has no ghost components), it follows that the moduli space $\mathcal{M}_{k+\ell_{0}+\ell_{1}}$ $\left(A, K ; Y_{0} \cup Y_{1}\right)$ is a smooth manifold of dimension

$$
\operatorname{dim}_{\mathbb{R}} \mathcal{M}_{k+\ell_{0}+\ell_{1}}\left(A, K ; Y_{0} \cup Y_{1}\right)=2 n-6+2 k+2 c_{1}(A)=2 d .
$$

For the extension of Theorem 1.2 to the case of two hypersurfaces, we need to show that the evaluation map at the first $k$ points

$$
\mathrm{ev}^{k}: \mathcal{M}_{k+\ell_{0}+\ell_{1}}\left(A, K ; Y_{0} \cup Y_{1}\right) \longmapsto X^{k}
$$

is a $2 d$-dimensional pseudocycle. The key to the proof is the following result which we will apply in the cases $I=\left\{k+1, \ldots, k+\ell_{0}\right\}$ and $I=\{k+1, \ldots, k+$ $\left.\ell_{0}+\ell_{1}\right\}$.

Proposition 9.10. For $\left\{k+1, \ldots, k+\ell_{0}\right\} \subset I \subset\left\{k+1, \ldots, k+\ell_{0}+\ell_{1}\right\}$ let $K \in \mathcal{J}_{|I|+1}^{*}\left(X, Y_{0} \cup Y_{1} ; J_{0}, \theta_{1}\right), k \geq 0$ and $A \in H_{2}(X, \mathbb{Z})$ with $\ell_{i}=D_{i} \omega(A)$. Suppose that a sequence $\left[f^{\nu}\right] \in \mathcal{M}_{k+\ell_{0}+\ell_{1}}\left(A, K ; Y_{0} \cup Y_{1}\right)$ converges in the sense of Gromov to a stable map $[\mathbf{z}, \mathbf{f}]$. Then the nodal curve $\mathbf{z}$ is I-stable. 
Proof. Arguing by contradiction, suppose that $\mathbf{z}$ is not $I$-stable. Then there exists a nonconstant component $f_{\alpha}$ of $\mathbf{f}$ whose domain $S_{\alpha}$ carries at most two special points after removing the marked points not belonging to $I$.

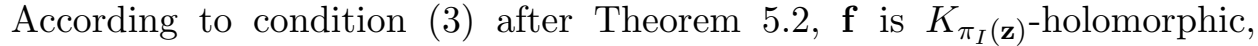
where $\pi_{I}$ is defined by removing the marked points outside $I$ and then stabilizing. As $\pi_{I}$ maps $S_{\alpha}$ to a point, this means that $\bar{\partial}_{K_{\alpha}} f_{\alpha}=0$ for some $K_{\alpha} \in \mathcal{J}^{*}\left(X, Y_{0} \cup Y_{1} ; J_{0}, \theta_{1}, E_{|I|}\right)$ (not depending on points on $S_{\alpha}$ ), where $E_{|I|}=|I| / \min \left(D_{0}, D_{1}\right)$.

By condition (a) in Definition 8.19 (since $f_{\alpha}$ is nonconstant), $f_{\alpha}$ is not contained in $Y_{0} \cup Y_{1}$, and by condition (b) it intersects $Y_{0}$ in at least three points in the domain. Thus $f_{\alpha}$ must intersect $Y_{0}$ at a point that is neither a node nor one of the middle $\ell_{0}$ marked points. By Proposition 7.1, intersections persist under small perturbations. So the definition of Gromov convergence implies that for $\nu$ sufficiently large, $\mathbf{f}^{\nu}$ intersects $Y_{0}$ at a point which is not one of the middle $\ell_{0}$ marked points. On the other hand, by Proposition 7.1 each of the middle $\ell_{0}$ marked points contributes at least 1 to the intersection number (since $f_{\nu}: S^{2} \rightarrow X$ is a smooth $K$-holomorphic curve with distinct marked points). Hence we conclude $\left[Y_{0}\right] \cdot\left[\mathbf{f}^{\nu}\right]>\ell_{0}=\left[Y_{0}\right] \cdot A$, in contradiction to $\left[\mathbf{f}^{\nu}\right]=A$.

Proposition 9.11. For $\left\{k+1, \ldots, k+\ell_{0}\right\} \subset I \subset\left\{k+1, \ldots, k+\ell_{0}+\ell_{1}\right\}$ and all $K \in \mathcal{J}_{|I|+1}^{\mathrm{reg}}\left(X, Y_{0} \cup Y_{1} ; J_{0}, \theta_{1}\right)$ (as defined above) the following holds. Let $A \in H_{2}(X ; \mathbb{Z})$ with $\omega(A)>0, \ell_{i}=D_{i} \omega(A), T$ an I-stable $\left(k+\ell_{0}+\ell_{1}\right)$-labelled tree with more than one vertex, and $A_{\alpha} \in H_{2}(X ; \mathbb{Z})$ for $\alpha \in T$ with $\sum A_{\alpha}=A$. Then the evaluation map $\mathrm{ev}^{k}: \mathcal{M}_{T}\left(\left\{A_{\alpha}\right\}, K ; Y_{0} \cup Y_{1}\right) \rightarrow X^{k}$ factors through a manifold of dimension at most $2 d-2$.

Proof. If every ghost tree in $T$ contains at most one of the last $\ell$ marked points the conclusion of the proposition follows from Theorem 9.8 because $e(T) \geq 1$ if $T$ has more than one vertex.

It remains to analyze the case that there exists a ghost tree $T^{\prime} \subset T$ containing at least two of the last $\ell$ marked points. Let $R$ be the reduced index set (retaining only the point with maximal index $i$ on each ghost tree) and $\bar{T}:=\pi_{R}(T)$ the tree obtained by removing the marked points outside $R$ and stabilizing. By Proposition 9.1(a), the evaluation map ev ${ }^{k}$ factors through the manifold $\mathcal{M}_{\bar{T}}^{*}\left(\left\{A_{\alpha}\right\}, K ;\left\{Z_{i}\right\}\right)$, where $Z_{i}=X$ for $i=1, \ldots, k, Z_{i}=Y_{0}$ for $i=k+1, \ldots, k+\ell_{0}$, and $Z_{i}=Y_{1}$ for $i=k+\ell_{0}+1, \ldots, k+\ell$. Now we distinguish two cases.

Case 1. $\bar{T}$ has more than one vertex. Then by Proposition 9.1(a), the evaluation map ev ${ }^{k}$ factors through the moduli space $\mathcal{M}_{\bar{T}}^{*}\left(\left\{A_{\alpha}\right\}, K ;\left\{Z_{i}\right\}\right)$ of dimension at most $2 d-e(\bar{T}) \leq 2 d-2$. 
Case 2. $\bar{T}$ has only one vertex. This happens iff $A_{\alpha} \neq 0$ for exactly one vertex $\alpha$, so we are in the situation of Proposition 9.1(b). Now recall that by assumption $T$ had at least two of the last $\ell$ marked points on the same ghost tree $T^{\prime}$. There are three subcases.

Case 2(a). $T^{\prime}$ contains two of the middle $\ell_{0}$ marked points. Then Lemma 7.2 shows that for each $(\mathbf{z}, \mathbf{f}) \in \mathcal{M}_{T}\left(\left\{A_{\alpha}\right\}, K ; Y_{0} \cup Y_{1}\right)$ the map $f_{\alpha}$ has local intersection number with $Y_{0}$ at least 2 (hence order of tangency at least 1) at the marked point $z_{\alpha j}, k+1 \leq j \leq k+\ell_{0}$, at which the ghost tree $T^{\prime}$ is attached. Hence by Proposition 9.1(b) the evaluation map ev ${ }^{k}$ factors through the moduli space $\mathcal{M}_{|R|}^{*}\left(A_{\alpha}, K ;\left\{Z_{i}, \ell_{i}\right\}\right)$, where $Z_{i}=Y_{0}$ or $Y_{1}$ and $\ell_{i} \geq 0$ for all $i \geq k+1$, and $\ell_{j} \geq 1$. So the dimension formula in Proposition 9.1(b) shows that

$$
\operatorname{dim} \mathcal{M}_{|R|}^{*}\left(A_{\alpha}, K ;\left\{Z_{i}, \ell_{i}\right\}\right) \leq 2 d-2 \sum_{i \in R, i \geq k+1} \ell_{i} \operatorname{codim}_{\mathbb{C}} Z_{i} \leq 2 d-2 .
$$

Case 2(b). $T^{\prime}$ contains two of the last $\ell_{1}$ marked points. This is analogous to Case 2(a).

Case 2(c). $T^{\prime}$ contains one of the middle $\ell_{0}$ and one of the last $\ell_{1}$ marked points. Then for each $(\mathbf{z}, \mathbf{f}) \in \mathcal{M}_{T}\left(\left\{A_{\alpha}\right\}, K ; Y_{0} \cup Y_{1}\right)$ the map $f_{\alpha}$ maps the marked point $z_{\alpha j}, k+1 \leq j \leq k+\ell_{0}$, at which the ghost tree $T^{\prime}$ is attached to the intersection $Y_{0} \cap Y_{1}$. Hence by Proposition 9.1(a) the evaluation map ev ${ }^{k}$ factors through the moduli space $\mathcal{M}_{|R|}^{*}\left(A_{\alpha}, K ;\left\{Z_{i}\right\}\right)$, where $Z_{j}=Y_{0} \cap Y_{1}$ and $Z_{i}=Y_{0}$ or $Y_{1}$ for $j \neq i \geq k+1$. So the dimension formula in Proposition 9.1(a) shows that

$$
\begin{aligned}
\operatorname{dim} \mathcal{M}_{|R|}^{*}\left(A_{\alpha}, K ;\left\{Z_{i}\right\}\right) \leq & 2 n-6+2 c_{1}(A)+2|R| \\
& -2 \sum_{i \in R} \operatorname{codim}_{\mathbb{C}} Z_{i} \leq 2 d-2 .
\end{aligned}
$$

This proves Proposition 9.11.

Propositions 9.10, 9.11 and the definition of a pseudo-cycle immediately imply the following version of Theorem 1.2 for two hypersurfaces.

Theorem 9.12. In the notation of Theorem 9.8, for every $k \geq 1$ the evaluation map at the first $k$ points

$$
\mathrm{ev}^{k}: \mathcal{M}_{k+\ell_{0}+\ell_{1}}\left(A, K ; Y_{0} \cup Y_{1}\right) \longmapsto X^{k}
$$

represents a pseudocycle of dimension

$$
2 d:=2\left(n-3+k+c_{1}(A)\right) .
$$




\section{Independence of the auxiliary data}

In this section, we prove that different choices of the auxiliary data $(Y, J ; K)$, where $(J, Y)$ is a Donaldson pair and $K \in \mathcal{J}_{\ell+1}^{\text {reg }}\left(X, Y ; J, \theta_{1}\right)$, give rise to rational pseudo-cycles which are rationally cobordant.

Throughout this section we fix the following data:

- a symplectic manifold $(X, \omega)$ with integral class $[\omega]$;

- a homology class $A \in H_{2}(X ; \mathbb{Z})$ with $0<\omega(A)$;

- an integer $k \geq 1$;

- a constant $\varepsilon>0$ and constants $0<\theta_{3}<\theta_{2}<\theta_{1}<\theta_{0}<1$ as in Lemmas 8.9 and 8.7(b);

- a closed 1-form $\alpha$ representing the first Chern class of $X$.

As in the previous section, by a Donaldson pair we understand a pair $(J, Y)$, where

- $J$ is an $\omega$-compatible almost complex structure on $X$;

- $Y \subset X$ is a hypersurface whose Kähler angle with respect to $(\omega, J)$ satisfies $\theta(Y ; \omega, J)<\theta_{3}$ and such that $[Y]=D[\omega]$ with $D \geq D^{*}(X, \omega, J)$, where $D^{*}(X, \omega, J)$ is the constant defined in Section 9 .

We always set $\ell:=D \omega(A)$. Theorem 1.2 assigns to each Donaldson pair $(J, Y)$ and domain-dependent perturbation $K \in \mathcal{J}_{\ell+1}^{\text {reg }}\left(X, Y ; J, \theta_{1}\right)$ in the Baire set of Theorem 1.1 a pseudo-cycle

$$
\operatorname{ev}^{k}(J, Y ; K): \mathcal{M}_{k+\ell}(A, K ; Y) \longrightarrow X^{k} .
$$

We first prove independence of the perturbation $K$.

Proposition 10.1. For a Donaldson pair $(J, Y)$ and $K_{0}, K_{1} \in \mathcal{J}_{\ell+1}^{\mathrm{reg}}(X, Y$; $\left.J, \theta_{1}\right)$, the pseudo-cycles

$$
\operatorname{ev}_{i}^{k}\left(J, Y ; K_{i}\right): \mathcal{M}_{k+\ell}\left(A, K_{i} ; Y\right) \longrightarrow X^{k}, \quad i=0,1,
$$

are cobordant.

Proof. Note that we can connect $K_{0}, K_{1}$ by a smooth path $K_{t} \subset \mathcal{J}_{\ell+1}^{*}(X, Y$; $\left.J, \theta_{1}\right)$. Indeed, by the $\theta_{2}$-contractibility condition in Definition 9.4 we can connect the $K_{i}$ in $\mathcal{J}_{\ell+1}^{*}\left(X, Y ; J, \theta_{1}\right)$ to domain-independent almost complex structures $K_{i}^{*} \in \mathcal{J}^{*}\left(X, Y ; J, \theta_{2}, E\right)$, and by Corollary 8.16 we can connect $K_{0}^{*}$ and $K_{1}^{*}$ by a path in $\mathcal{J}^{*}\left(X, Y ; J, \theta_{1}, E\right)$. Now the proof works exactly as in [19], repeating the arguments in Section 9 for a generic path $K_{t}$ in $\mathcal{J}_{\ell+1}^{*}\left(X, Y ; J, \theta_{1}\right)$.

As in the previous section, we denote by Donaldson quadruple a quadruple $\left(J_{0}, Y_{0}, J_{1}, Y_{1}\right)$ consisting of two Donaldson pairs $\left(J_{i}, Y_{i}\right)$ such that $Y_{0}, Y_{1}$ are $\varepsilon$-transverse and $\left\|J_{0}-J_{1}\right\|_{0}<\theta_{3}$. Theorem 9.12 associates a pseudo-cycle

$$
\operatorname{ev}^{k}\left(J_{0}, Y_{0}, J_{1}, Y_{1} ; K\right): \mathcal{M}_{k+\ell_{0}+\ell_{1}}\left(A, K ; Y_{0} \cup Y_{1}\right) \longrightarrow X^{k}
$$


to each Donaldson quadruple $\left(J_{0}, Y_{0}, J_{1}, Y_{1}\right)$ and domain-dependent perturbation $K \in \mathcal{J}_{\ell_{0}+\ell_{1}+1}^{\mathrm{reg}}\left(X, Y_{0} \cup Y_{1} ; J_{0}, \theta_{1}\right)$ in the Baire set of Theorem 9.8 with $\ell_{i}:=D_{i} \omega(A)$. Again, this pseudo-cycle does not depend on the perturbation $K$ :

Proposition 10.2. For a Donaldson quadruple $\left(J_{0}, Y_{0}, J_{1}, Y_{1}\right)$ and $K_{0}, K_{1} \in \mathcal{J}_{\ell_{0}+\ell_{1}+1}^{\mathrm{reg}}\left(X, Y_{0} \cup Y_{1} ; J_{0}, \theta_{1}\right)$, the pseudo-cycles

$$
\operatorname{ev}_{i}^{k}\left(J_{0}, Y_{0}, J_{1}, Y_{1} ; K_{i}\right): \mathcal{M}_{k+\ell_{0}+\ell_{1}}\left(A, K_{i} ; Y_{0} \cup Y_{1}\right) \longrightarrow X^{k}, \quad i=0,1,
$$

are cobordant.

Proof. As in the previous proof, by Definition 9.9 and Corollary 8.20 we can connect $K_{0}, K_{1}$ by a smooth path $K_{t}$ in $\mathcal{J}_{\ell_{0}+\ell_{1}+1}^{*}\left(X, Y_{0} \cup Y_{1} ; J_{0}, \theta_{0}\right)$ and the proof works as in $[\mathbf{1 9}]$, repeating the arguments in Section 9 for a generic path $K_{t}$.

In view of Propositions 10.1 and 10.2, we can drop the perturbation $K$ from our notation and denote by $\mathrm{ev}^{k}(J, Y)$, respectively, $\mathrm{ev}^{k}\left(J_{0}, Y_{0}, J_{1}, Y_{1}\right)$ the induced rational cobordism classes of pseudo-cycles.

The following result relates the rational pseudo-cycle associated to a Donaldson quadruple $\left(J_{0}, Y_{0}, J_{1}, Y_{1}\right)$ to those associated to the Donaldson pairs $\left(J_{i}, Y_{i}\right)$.

Proposition 10.3. For each Donaldson quadruple $\left(J_{0}, Y_{0}, J_{1}, Y_{1}\right)$ there exist rational cobordisms

$$
\frac{1}{\ell_{0} !} \operatorname{ev}^{k}\left(J_{0}, Y_{0}\right) \sim \frac{1}{\ell_{0} ! \ell_{1} !} \operatorname{ev}^{k}\left(J_{0}, Y_{0}, J_{1}, Y_{1}\right) \sim \frac{1}{\ell_{1} !} \operatorname{ev}^{k}\left(J_{1}, Y_{1}\right) .
$$

Proof. We prove the first equivalence, the second one being analogous. Choose a perturbation $K_{0} \in \mathcal{J}_{\ell_{0}+1}^{\text {reg }}\left(X, Y_{0} \cup Y_{1} ; J_{0}, \theta_{1}\right)$ in the Baire set of Theorem 9.8 depending only on the middle $\ell_{0}$ marked points. (This corresponds to taking the index set $I=\left\{1, \ldots, \ell_{0}\right\}$ in Theorem 9.8.) Then, in particular, we have $K_{0} \in \mathcal{J}_{\ell_{0}+1}^{\text {reg }}\left(X, Y_{0} ; J_{0}, \theta_{1}\right)$, and its pullback under the projection $\pi_{\ell_{0}}$ forgetting the last $\ell_{1}$ points satisfies $\pi_{\ell_{0}}^{*} K_{0} \in \mathcal{J}_{\ell_{0}+\ell_{1}+1}^{\text {reg }}\left(X, Y_{0} \cup Y_{1}\right.$; $\left.J_{0}, \theta_{1}\right)$. Thus $\pi_{\ell_{0}}$ induces a commutative diagram of pseudo-cycles.

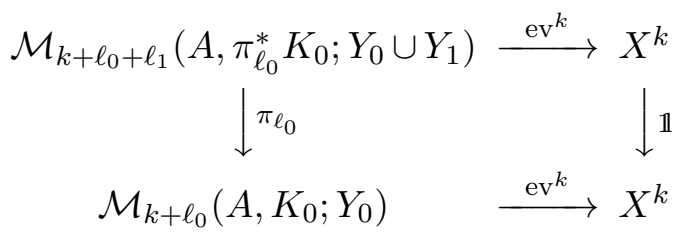

Now note that the moduli space $\mathcal{M}_{k+\ell_{0}+\ell_{1}}\left(A, \pi_{\ell_{0}}^{*} K_{0} ; Y_{0} \cup Y_{1}\right)$ is obtained from $\mathcal{M}_{k+\ell_{0}}\left(A, K_{0} ; Y_{0}\right)$ by simply adding the $\ell_{1}$ intersection points with $Y_{1}$ as additional marked points. (All these intersection points are distinct in the 
top stratum because any coincidence leads to a tangency to $Y_{1}$ and thus to a codimension 2 stratum.) Hence the map

$$
\pi_{\ell_{0}}: \mathcal{M}_{k+\ell_{0}+\ell_{1}}\left(A, \pi_{\ell_{0}}^{*} K_{0} ; Y_{0} \cup Y_{1}\right) \longrightarrow \mathcal{M}_{k+\ell_{0}}\left(A, K_{0} ; Y_{0}\right)
$$

is a covering map of degree $\ell_{1}$ ! and preceding commutative diagram yields the equality as currents

$$
\operatorname{ev}^{k}\left(J_{0}, Y_{0} ; K_{0}\right) \sim \frac{1}{\ell_{1} !} \operatorname{ev}^{k}\left(J_{0}, Y_{0}, J_{1}, Y_{1} ; \pi_{\ell_{0}}^{*} K_{0}\right) .
$$

In view of Propositions 10.1 and 10.2, this proves the first equivalence and hence Proposition 10.3.

The final ingredient is invariance under homotopies of Donaldson pairs.

Proposition 10.4. Let $\left(J_{t}, Y_{t}\right)_{t \in[0,1]}$ be a smooth family of Donaldson pairs. Suppose that $Y_{t}=\phi_{t}\left(Y_{0}\right)$ for a smooth family of symplectomorphisms $\phi_{t}$ with $\phi_{0}=11$. Then we have a rational cobordism of pseudo-cycles

$$
\operatorname{ev}^{k}\left(J_{0}, Y_{0}\right) \sim \mathrm{ev}^{k}\left(J_{1}, Y_{1}\right) .
$$

Proof. After cutting the interval $[0,1]$ into smaller ones, we may assume without loss of generality that $\left\|J_{0}-J_{1}\right\|$ is arbitrarily small and $\phi:=\phi_{1}$ is arbitrarily $C^{1}$-close to the identity. Then $\left\|\phi_{*} J_{0}-J_{1}\right\|$ will also be arbitrarily small.

Let $D$ be the degree of the $Y_{t}$ and $\ell:=D \omega(A)$. Choose $K_{0} \in \mathcal{J}_{\ell+1}^{\mathrm{reg}}\left(X, Y_{0} ; J_{0}\right.$, $\left.\theta_{1} / 2\right)$, so $\phi_{*} K_{0} \in \mathcal{J}_{\ell+1}^{\mathrm{reg}}\left(X, Y_{1} ; \phi_{*} J_{0}, \theta_{1} / 2\right)$. For $\left\|\phi_{*} J_{0}-J_{1}\right\|$ sufficiently small this implies $\phi_{*} K_{0} \in \mathcal{J}_{\ell+1}^{\text {reg }}\left(X, Y_{1} ; J_{1}, \theta_{1}\right)$. Composition with $\phi$ induces a commutative diagram of pseudo-cycles.

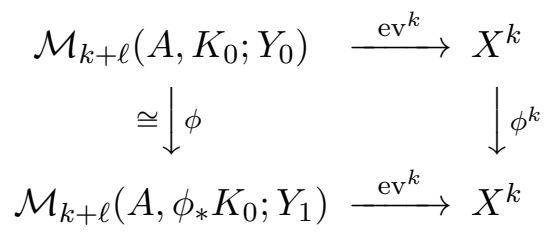

Since $\phi$ is smoothly homotopic to the identity, this implies the following rational cobordisms of pseudo-cycles:

$$
\operatorname{ev}^{k}\left(J_{0}, Y_{0} ; K_{0}\right) \sim \phi^{k} \circ \operatorname{ev}^{k}\left(J_{0}, Y_{0} ; K_{0}\right) \sim \operatorname{ev}^{k}\left(J_{1}, Y_{1} ; \phi_{*} K_{0}\right) .
$$

In view of Proposition 10.1, this proves Proposition 10.4.

Remark 10.5. Proposition 10.4 can also be proved, without using the symplectic isotopy $\phi_{t}$, by considering the moduli space $\cup_{t \in[0,1]} \mathcal{M}_{k+\ell}\left(A, K_{t} ; Y_{t}\right)$ for a generic family $\left(K_{t}\right)_{t \in[0,1]}$.

Proof of Theorem 1.3. Let $\left(J_{i}, Y_{i}\right), i=0,1$ be Donaldson pairs of degrees $D_{i}$ and set $\ell_{i}:=D_{i} \omega(A)$. Since $\theta\left(Y_{i} ; \omega, J_{i}\right)<\theta_{3}$, Lemma 8.5(c) provides compatible almost complex structures $\bar{J}_{i}$ preserving $T Y_{i}$ with $\left\|\bar{J}_{i}-J_{i}\right\|<\theta_{3}$. By the 
(Stabilization) statement in Theorem 8.1, for each sufficiently large integer $\bar{D}$ we find hypersurfaces $\bar{Y}_{i}$ of degree $\bar{D}$ such that $\left(J_{i}, Y_{i}, \bar{J}_{i}, \bar{Y}_{i}\right)$ are Donaldson quadruples for $i=0,1$. Set $\bar{\ell}:=\bar{D} \omega(A)$. Connect $\bar{J}_{0}$ and $\bar{J}_{1}$ by a smooth path of compatible almost complex structures $\bar{J}_{t}$. By the (Uniqueness) statement in Theorem 8.1, for $\bar{D}$ sufficiently large there exist smooth paths of hypersurfaces $\bar{Y}_{t}$ and symplectomorphisms $\phi_{t}$ with $\phi_{0}=1 \mathrm{l}$ such that the $\left(\bar{J}_{t}, \bar{Y}_{t}\right)$ are Donaldson pairs and $\bar{Y}_{t}=\phi_{t}\left(\bar{Y}_{0}\right)$ for all $t \in[0,1]$. Now Propositions 10.3 and 10.4 yield the following rational cobordisms of rational pseudo-cycles:

$$
\frac{1}{\ell_{0} !} \operatorname{ev}^{k}\left(J_{0}, Y_{0}\right) \sim \frac{1}{\bar{\ell} !} \operatorname{ev}^{k}\left(\bar{J}_{0}, \bar{Y}_{0}\right) \sim \frac{1}{\bar{\ell} !} \operatorname{ev}^{k}\left(\bar{J}_{1}, \bar{Y}_{1}\right) \sim \frac{1}{\ell_{1} !} \operatorname{ev}^{k}\left(J_{1}, Y_{1}\right) .
$$

This concludes the proof of Theorem 1.3.

\section{References}

[1] R. Adams, Sobolev spaces, Academic Press, 1975.

[2] D. Auroux, Asymptotically holomorphic families of symplectic submanifolds, Geom. Funct. Anal. 7 (1997), 971-995.

[3] D. Auroux, D. Gayet and J.-P. Mohsen, Symplectic hypersurfaces in the complement of an isotropic submanifold, Math. Ann. 321 (2001), 739-754.

[4] S. Donaldson, Symplectic submanifolds and almost-complex geometry, J. Diff. Geom. 44(4) (1996), 666-705.

[5] S. Donaldson, Lefschetz pencils on symplectic manifolds, J. Diff. Geom. 53(2) (1999), 205-236.

[6] N. Dunford and J.T. Schwartz, Linear operators part II, Spectral theory, self adjoint operators in Hilbert Spaces, Wiley \& Sons, New York, 1988.

[7] M. do Carmo, Differential forms and applications, Springer, Berlin, 1994.

[8] Y. Eliashberg, A. Givental and H. Hofer, Introduction to symplectic field theory, Geom. and Funct. Anal., Special Volume (2000), 560-673.

[9] A. Floer, The unregularized gradient flow of the symplectic action, Comm. Pure Appl. Math. 41(6), (1988), 775-813.

[10] A. Floer, H. Hofer and D. Salamon, Transversality in elliptic Morse theory for the symplectic action, Duke Math. J. 80(1) (1995), 251-292.

[11] K. Fukaya, Y.-G. Oh, H. Ohta and K. Ono, Lagrangian intersection Floer homology Anomaly and obstruction, Preprint, 2000, http://www.math.kyoto-u.ac.jp/ fukaya.

[12] K. Fukaya and K. Ono, Arnold conjecture and Gromov-Witten invariant, Topology 38(5) (1999), 933-1048.

[13] E. Giroux, Géométrie de contact: de la dimension trois vers les dimensions supérieures, Proceedings of the International Congress of Mathematicians, Vol. II (Beijing, 2002), Higher Ed. Press, Beijing, 2002, 405-414.

[14] E. Ionel and T. Parker, Relative Gromov-Witten invariants, Ann. of Math. (2) 157(1) (2003), 45-96.

[15] E. Ionel and T. Parker, The symplectic sum formula for Gromov-Witten invariants, Ann. of Math. (2) 159(3) (2004), 935-1025. 
[16] J. Li and G. Tian, Virtual moduli cycles and Gromov-Witten invariants of general symplectic manifolds, Topics in symplectic 4-manifolds (Irvine, CA, 1996), Int. Press, 1998, 47-83.

[17] G. Liu and G. Tian, Floer homology and Arnold conjecture, J. Diff. Geom. 49(1) (1998), 1-74.

[18] D. McDuff and D. Salamon, Introduction to symplectic topology, 2nd edn, Oxford University Press, New York, 1998.

[19] D. McDuff and D. Salamon, J-holomorphic curves and symplectic topology, Vol. 52, AMS Colloquium Publications, Providence, RI, 2004.

[20] Y. Ruan and G. Tian, A mathematical theory of quantum cohomology, J. Diff. Geom. 42(2) (1995), 259-367.

[21] W. Rudin, Functional analysis, McGraw-Hill, New York, 1973.

[22] B. Siebert, Symplectic Gromov-Witten invariants, New trends in algebraic geometry (Warwick, 1996), London Mathematical Society Lecture Note Series 264, Cambridge University Press, Cambridge, 1999, 375-424.

MAThematisches Institut

UNIVERSITÄT MÜNCHEN

D-80333 München, Germany

E-mail address: kai@mathematik.uni-muenchen.de

INSTITUT FÜR MATHEMATIK

Humboldt-Universität ZU BERLiN

D-10099 Berlin, Germany

E-mail address: mohnke@mathematik.hu-berlin.de

Received 12/29/2006, accepted 1/03/2008

The ideas for this paper emerged in discussions with Y. Eliashberg back in 2000. Over the subsequent years we benefited from discussions with many people including D. Auroux, D. McDuff, O. Fabert, E. Ionel, J. Latschev, D. Salamon and C. Stamm. Finally, we thank the referee for pointing out several gaps and errors in earlier versions. 